RECEIVED

AUG 281995

OSTI

\title{
Development of anAdvanced System Identification Technique for Comparing ADAMS Analytical Results with Modal Test Data for a Micon 65/13 Wind Turbine
}

Jan T. Bialasiewicz

NREL Technical Monitor:

Richard M. Osgood

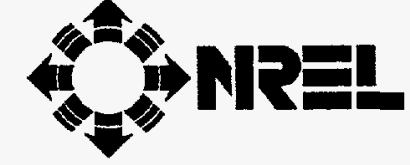

National Renewable Energy Laboratory 1617 Cole Boulevard Golden, Colorado 80401-3393

A national laboratory of the U.S. Department of Energy Managed by Midwest Research Institute for the U.S. Department of Energy under contract No. DE-AC36-83CH10093

Prepared under Subcontract No. CAT-5-15011-01

July 1995 


\section{NOTICE}

This report was prepared as an account of work sponsored by an agency of the United States government. Neither the United States government nor any agency thereof, nor any of their employees, makes any warranty, express or implied, or assumes any legal liability or responsibility for the accuracy, completeness, or usefulness of any information, apparatus, product, or process disclosed, or represents that its use would not infinge privately owned rights. Reference herein to any specific commercial product, process, or service by trade name, trademark, manufacturer, or otherwise does not necessarily constitute or imply its endorsement, recommendation, or favoring by the United States government or any agency thereof. The views and opinions of authors expressed herein do not necessarily state or reflect those of the United States government or any agency thereof.

Available to DOE and DOE contractors from:

Office of Scientific and Technical Information (OSTI)

P.O. Box 62

Oak Ridge, TN 37831

Prices available by calling (615) $576-8401$

Available to the public from:

National Technical information Service (NTIS)

U.S. Department of Commerce

5285 Port Royal Road

Springfield, VA 22161

(703) $487-4650$ 


\section{DISCLAIMER}

Portions of this document may be illegible in electronic image products. Images are produced from the best available original document. 


\section{Foreword}

This work uses the theory developed in the previous study, Advanced System Identification Techniques for Wind Turbine Structures with Special Emphasis on Modal Parameters, to analyze simulated data from an $\mathrm{ADAMS}^{\circ}$ (Automated Dynamic Analysis of Mechanical Systems) model of the MICON 65/13 wind turbine. The Observer/Kalman Filter identification approach delineated in the previous work is expanded to use input-output time histories from ADAMS simulations or structural test data. The purpose of the current work is to develop a methodology based on a state-space model of the system for using experimental data to systematically adjust the analytical model's structural parameters. Wind turbine analysts have used computers to model subsystems and entire wind turbine systems. A full system model of a wind turbine is most effectively built-up from smaller subsystem models that have been validated against structural test results. A common way researchers validate an analytical model is to compare the predicted with measured natural frequencies, damping ratios, and mode shapes. However, adjusting an analytical model's structural parameters so that it correctly predicts the wind turbine's dynamic response can be time-consuming and tedious work. The task of tuning an ADAMS model of a wind turbine can be further complicated by naturally occurring dissimilarities between the experimental test setup and assumptions made in developing an analytical model of the structure.

We use an ADAMS model of the MICON 65/13 wind turbine system with a set of SERI thin-airfoil blades. The ADAMS model was developed in April 1993 by Marshall Buhl, Senior Research Engineer for National Renewable Energy Laboratory's Wind Technology Division. A modal survey of this wind system was conducted in January of 1990 at SeaWest's windpark in Palm Springs, California. Although time histories were not measured during the modal test, frequency response functions were recorded. We computed from the measured frequency response functions their corresponding impulse response functions to obtain time histories of the response of the structure to an impulse. This work provides an example of using a common basis with which to compare experimental results with an analytical model's predictions. The advanced system identification technique uses experimental and analytical time histories to build two separate state-space models of the structure; one model for each source of input-output data. Once a state-space model of the structure is available, the identification technique computes the system's natural frequencies, damping ratios, and mode shapes. The dynamist can then compare the analytical and experimental state-space models' differences in calculated modal parameters to help determine how to adjust the ADAMS model.

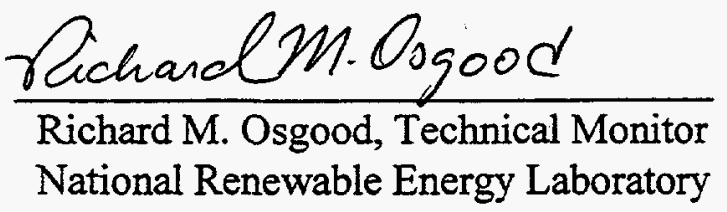




\section{Contents}

Page

1.0

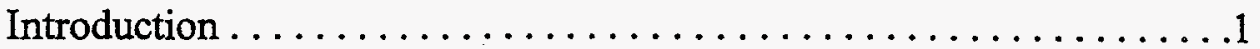

2.0

Modified Observer/Kalman Filter IdentificationTechnique . . . . . . . . 2

2.1 Selection of Input Signal . . . . . . . . . . . . . . . . . . 2

2.2 Selection of Measurements to be Obtained from a Modal Test . . . 5

2.3 Obtaining Wind Turbine State Space Model ... . . . . . . . . . . 9

3.0 Tools for Validation of the ADAMS Model with Experimental

Modal Test Data . . . . . . . . . . . . . . . . . . . . . . . . . . . 12

3.1 Methodology of the Approach .

12

3.2 Results of Modal Parameter Identification Using Input/Output

Time -Series Obtained as Simulation Data . . . . . . . . . . 13

4.0 Capabilities of the Developed Identification Technique and Future

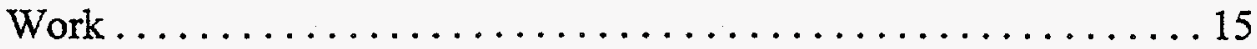

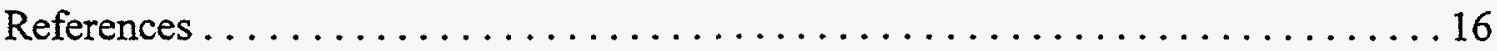

Appendix A Simulation/Identification Programs $\ldots \ldots \ldots \ldots \ldots \ldots \ldots \ldots$ A-1

Appendix B Application Notes and Identification Results Obtained Using

ADAMS Simulation Data for the Wind Turbine Micon $65 / 13 \ldots \ldots$. . B-1 


\section{List of Figures}

Page

A typical PRBS waveform .................... 3

$2-2$

Micon 65/13 Wind Turbine with virtual accelerometers and shakers. .6

\section{List of Tables}

Page

List of Measurement Points or Accelerometers ......... 7

$2-2$

Coordinates for Accelerometers on Micon $65 / 13 \ldots \ldots \ldots \ldots 8$

$2-3$

Out-of-plane Measurements . . . . . . . . . . . . . . . . . . 9

$2-4$

In-plane Measurements . . . . . . . . . . . . . . . . . 9

$3-1$

Modal Frequencies for Micon $65 / 13 \ldots \ldots \ldots \ldots \ldots \ldots 14$ 


\subsection{Introduction}

This research is a continuation of the previous study reported in [1]. The goal of the current research is to develop advanced system identification techniques for validating analytical models with experimental modal test data. Section 2.0 focuses on the modified observer/Kalman filter identification technique.

In Section 2.1, we discuss how the excitation or input signal should be selected. It is assumed that the input/output time series are available for both the simulated and real wind turbine tower structures. The input signal is assumed to be frequency rich, possibly identical for both the simulated and real structure, and applied at the same properly chosen driving point. It is proposed to use as the PRBS (Pseudo-Random Binary Sequence) signal with properly selected parameters in order to excite the structure in the desired bandwidth.

Then, in Section 2.2, we present the problem of making a proper selection of measurements to be obtained from a modal test. We illustrate this task by using sets of measurements for different excitation points that were obtained from a few simulation runs. Our conclusion is that for better accuracy. and verification of the results, several experiments/simulation runs should be performed using different driving points and/or measurements. In this research, we used four sets of measurements for excitations applied at different points of the ADAMS model of the Micon 65/13 turbine tower structure.

In Section 2.3, we introduce the Observer/Kalman Filter state space model obtained through identification of its Markov parameters and subsequent application of the Eigensystem Realization Algorithm, developed at NASA Langley Research Center [2-5]. The identification is performed by the modified MATLAB program fiokuy.m, originally introduced in the 1994 report [1].

In Section 3.1, we offer a step-by-step outline on how the tools developed in this research, can be used for validation of the ADAMS wind-turbine structure model with experimental modal test data.

In Section 3.2, we present general information on the tests performed using input/output time-series obtained from the ADAMS model of the wind turbine Micon 65/13 and we refer the interested reader to Appendix B for the identification results.

All MATLAB programs developed in this research are listed and described in Appendix A. An analysis of all the capabilities of the developed identification software tools, as well as an example of their application, are presented in Appendix B. Our conclusion is that the scope of applied research that this software could support is very broad. 


\subsection{Modified Observer/Kalman Filter Identification Technique}

\subsection{Selection of Input Signal}

Both the simulation and the experiment provide sampled input/output data. The sampling interval $T$ has to be properly chosen. The Nyquist frequency

$$
\omega_{N}=\frac{\pi}{T}[\mathrm{rad} / \mathrm{s}] \text { or } f_{N}=\frac{1}{2 T}[\mathrm{~Hz}]
$$

must be greater than the bandwidth of interest $f_{\max }$ of the structure. If the structure bandwidth considered is limited by the frequency $f_{\max }$, then (according to the sampling theorem) discrete-time representation of this process requires a sampling frequency of $f_{s}>2 f_{\max }$. The rule of thumb is to choose $f_{s}=(6$ to 25$) f_{\max }$ with $f_{s}=1 / T$ as high as possible. On the other hand, in order to correctly identify the steady-state gain of the process, the duration of at least one of the pulses in the PRBS must be greater than the rise time $t_{R}$ of the process.

The value of the input PRBS signal can change every $T_{p r b s}$ seconds; i.e, $T_{p r b s}$ is the switching period. A typical PRBS signal is shown in Fig.2.1. As shown in [7], the spectrum of the pseudo-random binary signal is an approximation for broad band noise, provided that its clock frequency is fast enough and its sequence length is large enough. The PRBSs are generated by means of shift registers with feedback (implemented in hardware or software). The maximum length $L$ of a sequence is

$$
L=2^{N}-1
$$

where $N$ is the number of stages of the shift register.

Because the maximum duration of a pulse is $N T_{p r b s}$, we have the following inequality:

$$
N T_{p r b s}>t_{R}
$$

Therefore, the clock frequency $f_{\text {prbs }}$ for the PRBS must be chosen as a submultiple of the sampling frequency $f_{s}$. If $f_{p r b s}=f_{S} / p(p=1,2, \ldots)$, then $T_{p r b s}=T p$ and, combining this with the inequality (2-3), we obtain the following condition that must be fulfilled:

$$
N>\frac{t_{R}}{T p}=\frac{t_{R}}{T_{p r b s}}
$$

Because lowering the clock frequency of the PRBS will reduce the frequency range in which its spectral density can be considered constant, we recommend choosing $p \leq 4$ [6]. 


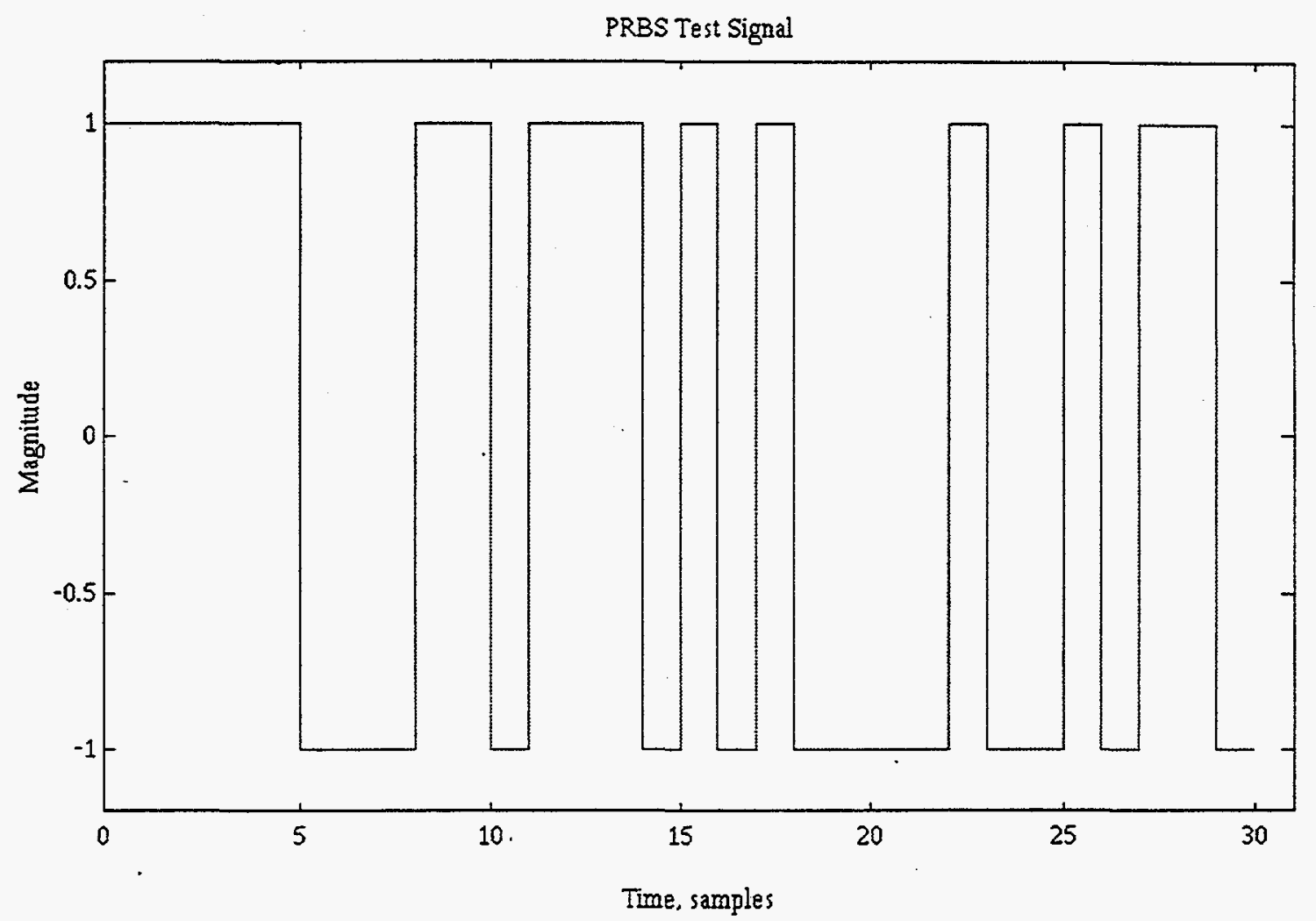

Figure 2-1 A typical PRBS waveform. 
The proper signal/noise ratio,i.e., at least 10:1, should be maintained even when magnitude $a$ of PRBS is very low. The output variations due to PRBS should be larger than the residual noise level. Also, for a low signal/noise ratio, it is necessary to extend the test duration in order to obtain satisfactory parameter estimates. The significant increase in the PRBS level may be undesirable because of plant nonlinearities.

Let us assume that the process to be identified has the banwidth of $5 \mathrm{~Hz}$, but at the same time, the bandwidth of interest is much higher, i.e., it is required to identify its higher order dynamics with modal frequencies above $5 \mathrm{~Hz}$. The time constant $\tau$ of such a process is approximately equal to $0.2 \mathrm{~s}$ and the rise time $t_{R} \cong 2 \tau=0.4 \mathrm{~s}$. We request that $N T_{\text {pros }}>t_{R}$. Therefore, if we set $N=10$, we should have $T_{p r b s}=p T>0.04$. Because we want the sampling interval to be as small as practical, we shall choose $p=4$ and $T=0.01$. This results in Nyquist frequency $f_{N}=50 \mathrm{~Hz}$ and we can expect accurate identification of the modal frequencies lower than $10 \mathrm{~Hz}$. Higher modal frequencies will be identified with some distortions. If the required bandwidth of interest is $30 \mathrm{~Hz}$, then, for accurate identification, the sampling frequency should at least be $180 \mathrm{~Hz}$ or the sampling period should be approximately $0.005 \mathrm{~s}$ to give $f_{N}=100 \mathrm{~Hz}$. On the other hand, assuming that the PRBS input sequence is generated with $N=10$ and $p=4$ (or is $p\left(2^{N}-1\right)=4092$ samples long), the maximum duration of a pulse in the input signal is $p N T=0.2 \mathrm{~s}$. Such an input sequence will not properly excite low-frequency modes, resulting in considerable distortions in the low-frequency range of the identified frequency response. A practical solution is to run several experiments with different sampling intervals and to obtain for each of them the frequency response accurate in a particular frequency range. Also, because of the noise presence, improving identification results may require the input sequence to be repeated several times during one experiment involving either the simulation or real process measurements run. To obtain the simulation data for the ADAMS model of the turbine Micon 65/13 used in this research, the input PRBS sequence (with $N=10$ and $p=$ 4) was repeated three times, i.e., the input-output time series had 12,276 points for each run. It turned out that, for every identification run, the identification error stabilizes at a low level after approximately 2500 samples (see appropriate plots in Appendix B). Therefore, the length of the input-output time series has been reduced to 4092 samples, resulting in a shorter identification process and still allowing for accurate identification of the low frequency modes.

The input signal was generated by the MATLAB program prbsmp.m which is included in Appendix A. Program users choose the magnitude $a$ and the number of stages $N$ of the shift register, which can be equal to any integer between 4 and 10 inclusive. Program users also choose the multiplier $p$ as any integer between 1 and 4 . As shown above, this allows for a small sampling interval $T$ (large Nyquist frequency) and $p$ times larger $T_{p r b s}=$ $p T$, which improves identification accuracy in the low frequency range. The MATLAB program prbsmp.m automatically plots the generated PRBS and stores the sequence in the PRB vector. 


\subsection{Selection of Measurements to be Obtained from a Modal Test}

The problem of the proper selection of measurements has been studied using simulation data for the ADAMS analytical model of the Micon 65/13 wind turbine structure, which is shown in Fig. 2.2. In this figure, all 32 measurement points are marked along the structure, but at each of them, we have two virtual accelerometers measuring in two directions: $\mathrm{Y}$ and $\mathrm{Z}$, according to the local coordinate systems, which are different for each blade and the tower, as shown in the figure. The list of accelerometers used in the simulation is given in Table 2.1. Their locations (same location for a pair of accelerometers measuring in $\mathrm{Y}$ and $\mathrm{Z}$ direction), in ground coordinate system (see Fig. 2.2), are given in Table 2.2. The measurement matrix generated by the ADAMS model had 64 columns representing virtual accelerometers, each with $3 p\left(2^{N}-1\right)$ elements (rows) representing measurements at sampling instants, i.e., the PRBS signal of 4092 samples was repeated three times to give long enough input-output sequence. As explained previously, this number has been reduced to $p\left(2^{N}-1\right)=4092$ for $N=10$ and $p=4$. In other words, due to noise absence in the simulation environment, it was not necessary to repeat the input sequence several times. The extension of the input sequence would improve identification accuracy olny to a certain degree depending on the noise level. Unfortunately it is impossible to establish a quantitative relation between length of the input sequence and the required identification accuracy (at a given sigal/noise ratio).

In order to excite all the modes, the simulation has been performed for three out-of-plane excitations and one in-plane excitation, all of the same PRBS type. The out-of-plane excitations were the excitations $F_{Y}$ at the two blade tips (locations B1S13 and B2S13) and excitation $F_{Z}$ (location TOWER4). For each of these three inputs, we generated a measurement matrix. For identification purposes, and as a result of sensitivity analysis, we reduced the set of 64 measurement variables. We selected five variables, i.e., five outputs or five columns of the measurement matrix, the same for each of the three aforementioned driving points. These are the outputs listed in Table 2.3. We applied the in-plane excitation $F_{Y}$ at the tower location TOWER4. To see the difference in the frequency response, i.e., to see that the excitation and, consequently, the identification of different modes depends on both the driving point and the set of measurement points, we performed the identification twice, using the set of the out-of-plane measurement points in Table 2.3 and the set of in-plane measurement points listed in Table 2.4. Corresponding pairs of measurement points related to measuring acceleration in two different directions have the same locations on the wind turbine structure. The identification results are presented in Appendix B.

The important conclusion for modal testing on a real wind turbine structure is that in order to determine natural frequencies and damping ratios the number of measurement points can be substantially reduced. A properly selected driving point-measurement set leads to accurate identification. This statement is supported by comparing frequency responses for a given excitation point and different outputs. However, to avoid missing some of the modes in bandwidth of interest, the driving points have to be carefully selected. As can be seen from the plots shown in Appendix B for any driving point, the 


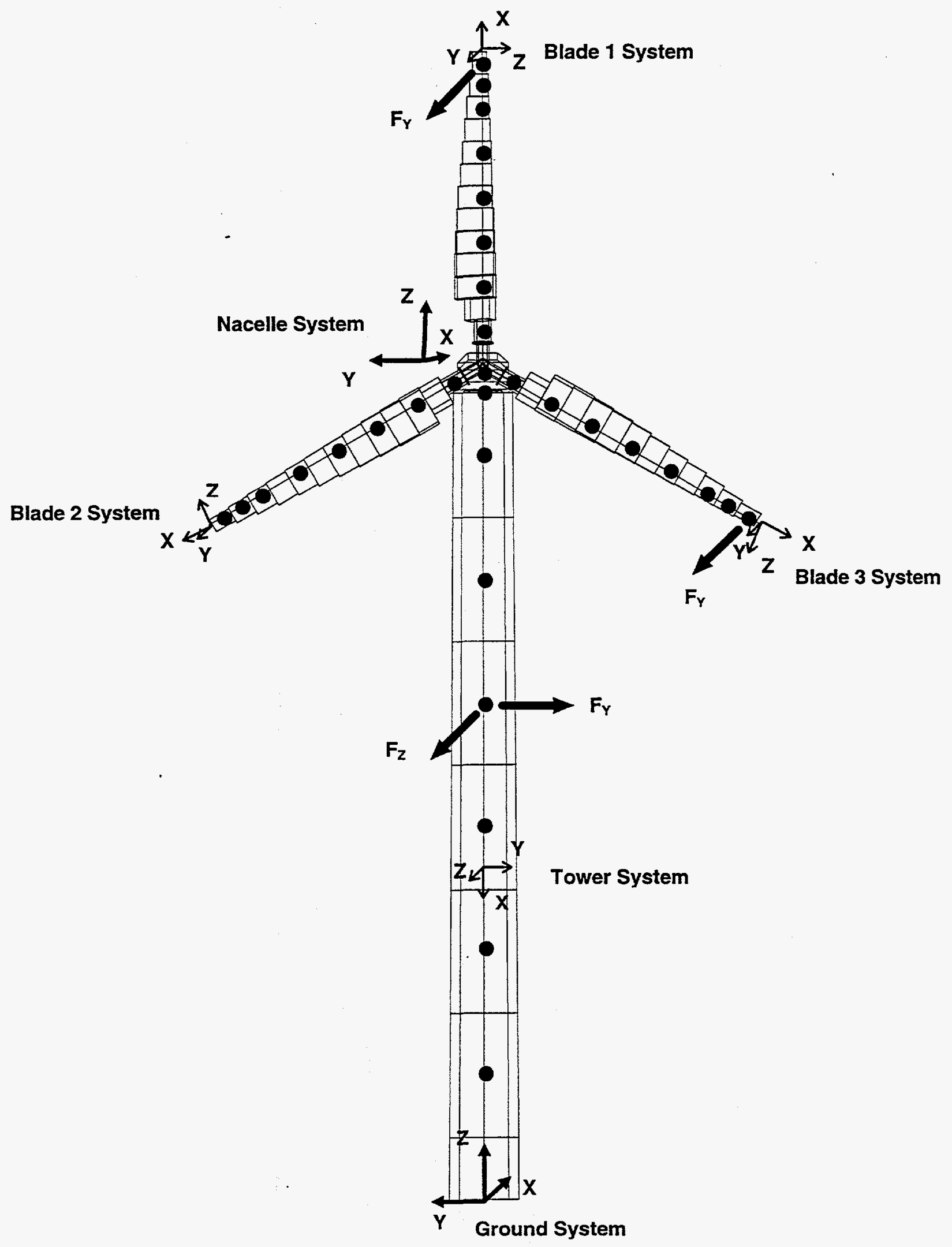

Figure 2.2. Micon 65/13 Wind Turbine with virtual accelerometers and shakers. 
frequency response for a collocated excitation-measurement pair gives the best resolution of the system's resonance modes.

\section{Table 2.1 List of Measurement Points}

\section{or Accelerometers}

Col Name

1 TOWER 1 Y

2 TOWER2_Y

3 TOWER3_Y

4 TOWER4 4 Y

5 TOWER5_Y

6 TOWER6_Y

7 SLRING_Y

8 NACEL_Y

9 TOWERI_Z

10 TOWER2_Z

11 TOWER3_Z

12 TOWER 4_Z

13 TOWER5_Z

14 TOWER6_Z

15 SLRING_Z

16 NACEI_ $\bar{X}$

17 B1S01_Y

18 B1S03_Y

19 B1S05_Y

20 B1S07_Y

21 B1S09_Y

22 B1S11_Y

23 B1S12_Y

24 B1S13-Y

25 B2S01_Y

26 B2SO3_Y

27 B2S05_Y

28 B2S07_Y

29 B2S09_Y

30 B2S11 Y

31 B2S12_Y

32 B2S13_Y

\section{Col Name}

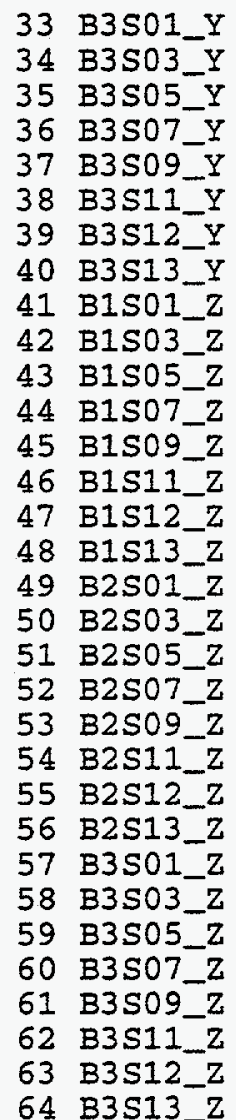


Table 2.2 Coordinates for accelerometers on Micon 65/13

Name Location in Ground System coordinates

( $X=$ downwind, $Y=$ orthogonal to $X-Z, Z=$ up ) as shown in Fig. 2.2.

\begin{tabular}{|c|c|c|}
\hline $\begin{array}{l}\text { TOWER1 } \\
\text { TOWER2 } \\
\text { TOWER3 } \\
\text { TOWER4 } \\
\text { TOWER5 } \\
\text { TOWER6 } \\
\text { SLRING }\end{array}$ & $\begin{array}{ccc}0, & -0, & 3.3780 \\
0, & 0, & 6.7620 \\
0, & 0, & 10.146 \\
0, & 0, & 13.531 \\
0, & 0, & 16.915 \\
0, & 0, & 20.300 \\
0, & 0, & 22.026\end{array}$ & \\
\hline NACEL & $0.071,0.055,22.444$ & \\
\hline $\begin{array}{l}\text { BISO1 } \\
\text { B1S03 } \\
\text { BISO5 } \\
B 1 S 07 \\
B 1 S 09 \\
B 1 S 11 \\
B 1 S 12 \\
B 1 S 13\end{array}$ & 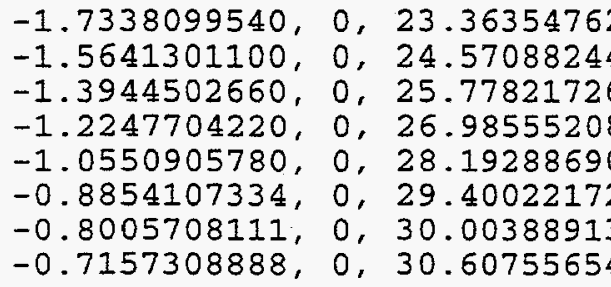 & \\
\hline $\begin{array}{l}B 2 S 01 \\
B 2 S 03 \\
B 2 S 05 \\
B 2 S 07 \\
B 2 S 09 \\
B 2 S 11 \\
B 2 S 12 \\
B 2 S 13\end{array}$ & 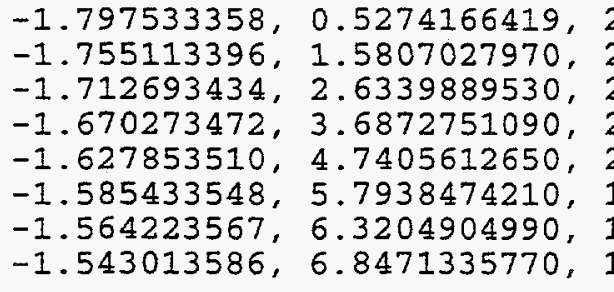 & $\begin{array}{l}22.45226046 \\
21.83969416 \\
21.22712786 \\
20.61456156 \\
20.00199526 \\
19.38942896 \\
19.08314581 \\
18.77686266\end{array}$ \\
\hline $\begin{array}{l}\text { B3SO1 } \\
\text { B3SO3 } \\
\text { B3SO5 } \\
\text { B3SO } \\
\text { B3SO9 } \\
\text { B3S11 } \\
B 3 S 12 \\
\text { B } 3 S 13\end{array}$ & 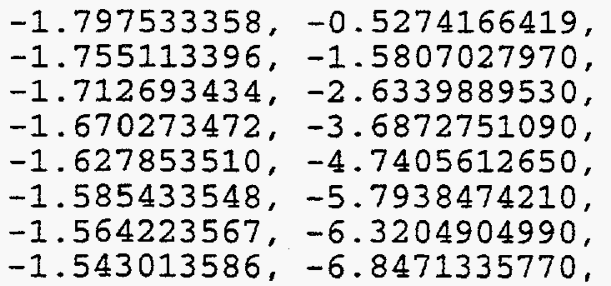 & $\begin{array}{l}22.45226046 \\
21.83969416 \\
21.22712786 \\
20.61456156 \\
20.00199526 \\
19.38942896 \\
19.08314581 \\
18.77686266\end{array}$ \\
\hline
\end{tabular}


Table 2.3 Out-of-plane measurements

\begin{tabular}{|c|c|c|c|c|c|c|c|c|}
\hline TOW & or & $\mathrm{Co}$ & 12 & OUTPUT 1 & TOWER4_Y & olumn & 4 & OUTPUT \\
\hline SLRI & or & column & 15 & OUTPUT 2 & SLRING_Y & or column & 7 & PUT \\
\hline B1S & or & column & 24 & OUTPUT 3 & $-\bar{z}$ & or & 48 & ot \\
\hline $\mathrm{B} 2$ & or & column & 32 & OUTPUT & 3_z & or & 56 & PUT \\
\hline & or & column & 40 & OUTPUT & B3S13_Z & olumn & 64 & OU: \\
\hline
\end{tabular}

\subsection{Obtaining Wind Turbine State Space Model}

The state space model is generated using a properly obtained input-output sequence. The generation of a proper input-output sequence has been previously discussed in Sections 2.1 and 2.2. The discrete-time state space model $(A, B, C, D)$, to be identified, defines the following relation between the scalar driving excitation $u(k)$ and the measurement $m$ vector (or output) $y(k)$ :

$$
\begin{array}{r}
x(k+1)=A x(k)+B u(k) \\
y(k)=C x(k)+D u(k)
\end{array}
$$

Note that this state space model depends on the choice of the state vector $x(t)$ and the sampling interval $T$. Assuming that $x(0)=0$ and solving for the system output, we obtain

$$
y(k)=\sum_{i=1}^{k} C A^{i-1} B u(k-i)+D u(k) .
$$

It can be easily shown that equation (2-6) represents the convolution of the system's input sequence $u(k)$ and the sequence $Y(k)$ with the following elements:

$$
Y_{0}=D, Y_{1}=C B, Y_{2}=C A B, \ldots, Y_{k}=C A^{k-1} B
$$

Therefore, these elements are consecutive samples of the system's pulse response and they are known as Markov parameters. Assuming that our input-output sequence has a length of $l$, we can write $l$ equations of the type of equation (2-6) with the number of terms on the right side increasing as the new input-output pairs become available. This set of $l$ equations can be represented by the following equation:

$$
[y(0) \vdots y(1) \vdots \ldots \vdots \mathrm{y}(l-1)]=\left[D \vdots C B: C A B \vdots \ldots \vdots C A^{l-2} B\right]\left[\begin{array}{r}
u(0) u(1) u(2) \ldots u(l-1) \\
u(0) u(1) \ldots u(l-2) \\
u(0) \ldots u(l-3) \\
\vdots \\
u(0)
\end{array}\right] \text {. }
$$


The wind turbine structure is a flexible structure with lightly' damped low frequency modes. For such a system, a sufficiently large number of equations $l=p$ is required to. have

$$
A^{k} \approx 0 \quad \text { for } \quad k \geq p
$$

This property holds for any stable system and $A^{k}$ for stable systems with higher damping approaches zero much faster. This signifies that to solve for the Markov parameters (as an adequate system representation) of a lightly-damped system a sufficiently large $l$ is required but for better damped systems the value of $l$ can be smaller.

A possible approach to solving this problem is to artificially increase system damping to obtain the solution for Markov parameters. The observer model of the system is used in this approach. The state equation (2-5) can be manipulated as follows:

$$
\begin{aligned}
x(k+1) & =A x(k)+B u(k)+G y(k)-G y(k) \\
& =(A+G C) x(k)+(B+G D) u(k)-G y(k)
\end{aligned}
$$

where $\mathrm{G}$ is an $n \times m$ arbitrary matrix chosen to make $A+G C$ as stable as desired. Equation (2-9) can be rewritten in a standard compact form:

$$
x(k+1)=\bar{A} x(k)+\bar{B} v(k)
$$

where

$$
\bar{A}=A+G C, \quad \bar{B}=[B+G D-G], \quad v(k)=\left[\begin{array}{l}
u(k) \\
y(k)
\end{array}\right] .
$$

Now, we can write an equation, similar to equation (2-8), but one which involves observer Markov parameters:

$$
\overline{\mathbf{Y}}=\left[D: C \bar{B}: C \overline{A B}: \ldots: \overline{C A}^{p-1} \bar{B}: \ldots \vdots \overline{C A}^{l-2} \bar{B}\right]
$$

For an observable system, we can assign the eigenvalues of $\bar{A}$ arbitrarily through a proper choice of $G$. In the case of the dead-beat observer, or when all the eigenvalues of $\bar{A}$ are placed at the origin, $C \bar{A}^{k} \bar{B} \approx 0$ for $k \geq p$ where $p$ is a sufficiently large integer. We can now solve for the observer Markov parameters

$$
\bar{Y}=\left[D: C \bar{B}: C \overline{A B}: \ldots: C \bar{A}^{p-1} \bar{B}\right]
$$

using a least-squares algorithm.

The observer Markov parameters in equation (2-12) include the system Markov parameters and the observer gain Markov parameters. The system Markov parameters are used to 
compute the system matrices $A, B, C$, and $D$, whereas the observer gain Markov parameters are used to determine the observer gain matrix $G$. The proper algorithm for obtaining these Markov parameters has been introduced by Phan et al. [5] and is also discussed by Juang [2]. The algorithm uses input and output time histories as its input. Software implementation of this identification algorithm was developed at NASA Langley and is known as the MATLAB function OKDD. Finally, the state-space representation $(A, B, C$, $D$ ) of the system is obtained using the Eigensystem Realization Algorithm (ERA), which is based on system realization theory.

It can be proven that the truncated observer model (2-12), obtained as a result of the deadbeat approximation of equation (2-10), produces the same input-output map as a Kalman filter if the data length is sufficient so that the truncation error is negligible. In this case, $G$ computed from combined Markov parameters of equation (2-12) gives the steady-state Kalman filter gain $K=-G$. 


\subsection{Tools for Validation of the ADAMS Model with Experimental Modal Test Data}

\subsection{Methodology of the Approach}

The ADAMS model of a wind turbine structure can be validated using experimental modal test data by processing (with the MATLAB programs developed in this research) the input-output time series obtained for both the real system and its ADAMS model. It is important that the input time series is properly chosen as outlined in Section 2.1, and that, as discussed in Section 2.2, this input signal is applied at different locations and the excitation signals are associated with properly selected sets of measurements. In other words, several properly designed experiments have to be performed. Also, if the frequency range of interest is relatively wide, it may be necessary to repeat each experiment for two or more $\left(T, T_{p r b s}\right)$ pairs to get good identification in both the low and the high frequency range. For the experiments reported in Appendix B, a single pair $\left(T, T_{p r b s}\right)=(0.01 \mathrm{~s}, 0.04 \mathrm{~s})$ was used. It is known that higher sampling frequency results in a higher Nyquist frequency allowing for better identification of higher frequency modes. However, as justified by our discussion in Section 2.1, $T_{\text {prbs }}$ is then too small to identify properly low frequency modes. Therefore, it is expected that if two pairs, such as $(0.02 \mathrm{~s}, 0.08 \mathrm{~s})$ and $(0.005 \mathrm{~s}, 0.02 \mathrm{~s})$, were used to run all the experiments twice, better identification accuracy would have been achieved. However, due to bandwidth limitations of hydraulic shakers the input-output time series generation for the second $\left(T, T_{p r o s}\right)$ pair and a real wind turbine structure, if possible at all, would be inaccurate.

The identification of the Observer/Kalman Filter model is performed by the MATLAB program fiokuy.m which uses the MATLAB function okid. The initial estimate of the number of observer Markov parameters has to be specified considering that the maximum system order that can be identified is the product $p \bullet m$. Using the measurement matrix, the Hankel matrix is formed and a plot of its singular values is shown to aid in selecting the correct system order. There is one singular value associated with each resonance mode. The fact that the mode's singular value is a measure of its contribution to the system's response aids in selection of the order of the system's model. After a particular order is selected, the percentage of data realized by the model is computed. It is recommended to choose the lowest system order resulting in $100 \%$ realization of the measurement data. Also, the corresponding modal parameters are displayed on the screen with the corresponding mode singular values (SV) and modal amplitude coherence (MAC) factors. This provides additional evaluation of the quality of the identified model. Looking at this table, the researcher can determine the modes whose contribution to the system dynamics is insignificant. Such modes can be classified as the noise modes.

The identified system matrices $A, B, C, D$, generated by the program for the structure model of a selected order, are available as MATLAB variables Af, Bf, Cf, Df. The identification error is displayed in the Figure Window. For the sake of validation of the $A D$ AMS model, we should run fiokuy.m for the experiments performed for both the real plant and its model. 
The next step is to run the identification programs idss2tfr.m and zoomtfr.m, for all input-output data files, with the number of times equal to the number of measurement points in the processed data file. The program idss 2 tfr.m displays identified eigenvalues and corresponding modal frequencies in [rad/s] and $[\mathrm{Hz}]$, as well as system zeros related to the selected output. The frequencies of the complex-conjugate zeros, which determine system's antiresonances, are also displayed. Program idss2tfr.m displays the frequency response in the Figure Window. Program zoomtfr.m can be used to enlarge a selected portion of this plot. Both programs use the state space model Af, Bf, Cf, Df, generated by the program fiokuy.m.

All the outlined steps of the above procedure are very well illustrated in Appendix B using simulation data obtained from the ADAMS model of the wind turbine Micon 65/13.

\subsection{Results of Modal Parameter Identification Using Input/Output Time-Series Obtained as Simulation Data}

The input/output time-series obtained from the ADAMS model of the Micon 65/13 wind turbine were used to develop and to validate the identification procedure presented above. It was found that to identify all vibration modes, we have to process, repeating the same procedure, the input/output time-series for both in-plane and out-of-plane excitations applied at various points of the wind turbine structure. This has been done for three data files generated by out-of-plane excitations, collocated with the measurements TOWER4_Z, B2S13_Y, and B1S13_Y, and for one data file generated by the in-plane excitation collocated with the measurement TOWER4_Y.

For each of the four data files listed above that contain five measurements, the $\mathrm{Ob}$ server/Kalman Filter state-space model has been identified interactively in order to determine the model order providing the best fit for the measurement data. The corresponding set of modal parameters has been generated. Then, for each of the five input-output pairs, the frequency response has been plotted and the corresponding set of system zeros and their frequencies has been determined.

Appendix B contains a detailed introduction presenting the scope of the tests performed. It also gives the numerical results of modal parameter identification, graphically illustrated by frequency response plots. This graphical illustration is most distinct on the frequency response plot for the system output (measurement) collocated with the excitation used to obtain the analyzed data file.

Modal frequencies identified from the simulation data using observer/Kalman filter identification technique along with the modal frequencies determined for the structure of $\mathrm{Mi}$ con 65/13 wind turbine from modal survey as well as those obtained using ADAMS linear model are given in Table 3.1. 
Table 3.1 Modal Frequencies (Hz) for Micon 65/13

\begin{tabular}{|l|c|c|c|}
\hline \multicolumn{1}{|c|}{ Bending Mode } & Modal Survey & ADAMS/Linear & OKID Technique \\
\hline 1st Tower/Aft & 1.60 & 1.65 & 1.72 \\
\hline 1st Tower Side-to-Side & 1.80 & 1.66 & 1.76 \\
\hline 1st Assymmetric 1st Flap & 2.70 & 3.18 & 2.91 \\
\hline 2nd Assymmetric 1st Flap & 3.20 & 3.21 & 3.22 \\
\hline 1st Symmetric 1st Flap & 3.60 & 3.36 & 3.49 \\
\hline 1st Symmetric 1st Edge & 6.70 & 7.48 & 7.10 \\
\hline 1st Assymmetric 1st Edge & 7.51 & 7.75 & 7.46 \\
\hline 2nd Assymmetric 1st Edge & 7.83 & 7.85 & 7.78 \\
\hline 1st Assymmetric 2nd Flap & 10.80 & 11.31 & 10.82 \\
\hline 2nd Assymmetric 2nd Flap & 11.10 & 11.34 & 11.08 \\
\hline 1st Symmetric 2nd Flap & 11.48 & 11.41 & 11.15 \\
\hline
\end{tabular}




\subsection{Capabilities of the Developed Identification Technique and Future Work}

An analysis of all the capabilities of the developed identification software tools indicates that the scope of applied research this software could support is very broad. The identification technique applied is capable of developing a multi-input multi-output model of a structure. Consequently, such a technique enables the researcher to handle data obtained from an experiment with more than one excitation point and, practically, unlimited number of measurement points.

The realization of a system obtained has a first-order or state space form and can be easily converted into the transfer function matrix form. This means that the transfer function between any excitation input (such as a force applied at the tip of one of the turbine blades) and any measurement output (such as acceleration at the top of the turbine tower) can be made available and its graphical representation in a form of the Bode plot can be obtained. Since each measurement is associated with particular geometrical coordinates or particular point on a structure, we can obtain at this point the magnitude and phase of a sinusoid of any modal frequency. They change along the structure resulting for each modal frequency in a particular mode shape.

The identification technique developed can help to adjust analytical model of a structure, such as ADAMS model. This can be done by comparing the predicted with identified natural frequencies, damping ratios and mode shapes, obtained using identification technique developed with modal test data. However, this comparison does not provide any analytical guidelines for tuning physical parameters of a lumped-parameter model formulated in terms of mass, damping, and stiffens matrices. Such a model is described by a set of $n / 2$ second-order differential equations with $n$ being the assumed (even) order of the system model. It is, therefore, necessary to replace an $n$ th-order state space representation by a properly structured representation which consists of $n / 2$ second-order equations. Additional information to be used comprises locations of the sensors and actuators.

Further applications of the identification technique developed and tested in this research using simulation data for the wind turbine Micon $65 / 13$ to be explored include modal testing of a rotating wind turbine and damage localization of a structure such as a wind turbine blade undergoing fatigue test, a building, an offshore platform or a highway bridge. In these prospective applications, data from the sensors installed can be processed periodically in order to determine the localization of a damage as well as the time at which it occurred. 


\section{REFERENCES}

[1] Bialasiewicz, J. T. (1994). Advanced System Identification Techniques for Wind Turbine Structures with Special Emphasis on Modal Parameters, TP-442-7110. Golden, CO: National Renewable Energy Laboratory.

[2] Juang, J.-N. 1994. Applied System Identification, Englewood Cliffs, New Jersey: Prentice Hall.

[3] Juang, J.-N.; Horta, L.G.; and Phan, M. (1992). System/Observer/Controller Identification Toolbox, NASA Technical Memorandum 107566, NASA Langley Research Center.

[4] Juang, J.-N.; Phan, M.; Horta, L.G.; and Longman, L.G. (1993). "Identification of Observer and Kalman Filter Markov Parameters: Theory and Experiments." Journal of Guidance, Control and Dynamics, Vol.16, No. 2, pp.320-329.

[5] Phan, M.; Horta, L.G.; Juang, J.-N.; and Longman, R.W. (1992). Linear System Identification Via an Asymptotically Stable Observer. NASA Technical Paper 3164.

[6] Landau, I.D. (1990). System Identification and Control Design. Englewood Cliffs, New Jersey: Prentice Hall.

[7] Newland, D.E. (1984). An Introduction to Random Vibrations and Spectral Analysis. New York: Longman. 


\section{APPENDIX A}

\section{Simulation/ldentification Programs}

\section{Program prbsmp.m}

This program has been developed to generate the frequency-rich input test signal to be used for the identification of dynamical systems. Its original version prbsm.m has been discussed in detail in [1] and the current modification is justified and described in Section 2.1 of this report. This program is listed below. It is an interactive program. The log of user-MATLAB interaction is as follows:

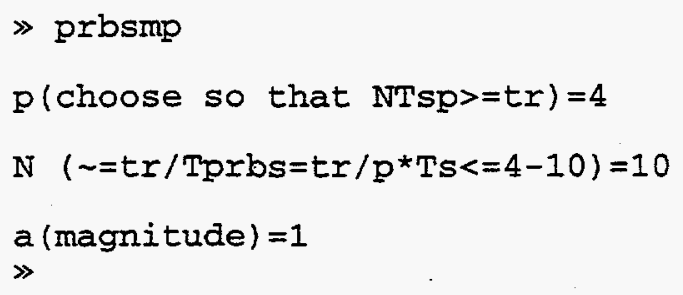

The user is asked to determine the magnitude $a$, the parameter $\mathrm{p}$, which sets the number of samples (or measurements) per $T_{p r b s}$, and the number of stages of the shift register $N$. The length of the generated sequence equals to $p\left(2^{N}-1\right)$. Also, the maximum pulse length in the sequence is equal to $N p$ clock (or sampling) periods. The sampling period has to be declared in the application program, which calls for execution of prbsmp.m. The generated PRBS sequence is available as a row vector PRB. The response to help prbsmp is as follows:

$\gg$ help prbsmp

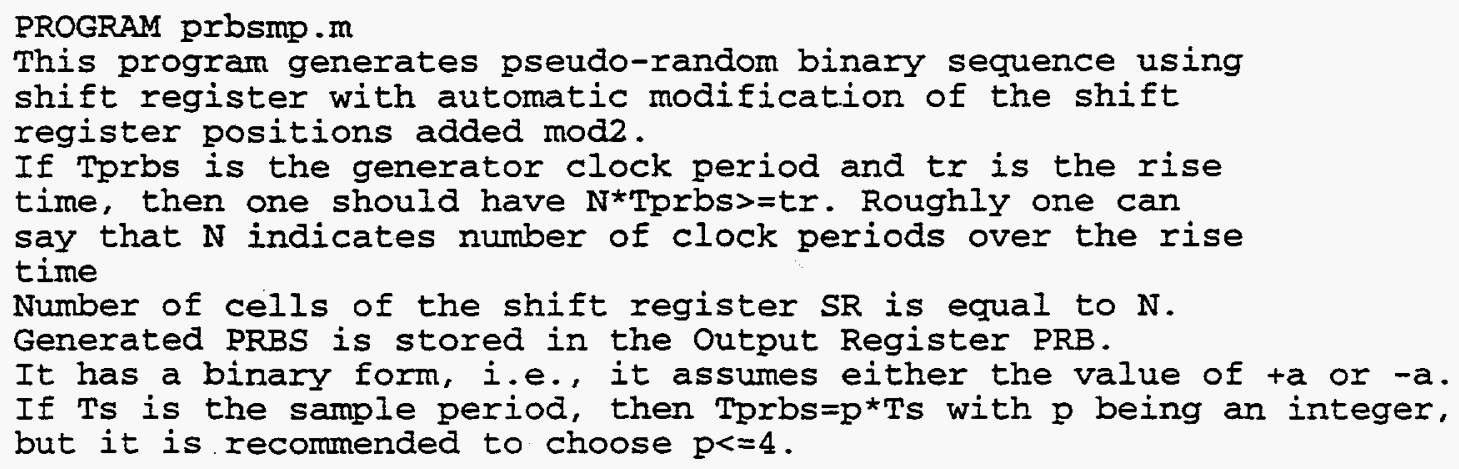

$\gg$

This information advises the user how the choice of $N$ and the generator clock period should be related to the dynamics of the system to be identified. For a linear system without noise, the magnitude $a$ of the excitation can have any value. For a real system, we have to consider the presence of noise and nonlinearities. We choose $a$ to achieve an acceptable signal-to-noise ratio but small enough in order to remain in the linear region of operation of a real system. 
The prbsmp.m listing is given below.

$\gg$ type prbsmp.m

\&PROGRAM prbsmp.m

\&This program generates pseudo-random binary sequence using

rshift register with automatic modification of the shift

sregister positions added mod2.

fIf Tprbs is the generator clock period and tr is the rise

otime, then one should have $N^{*}$ Tprbs $>=t r$. Roughly one can

osay that $N$ indicates number of clock periods over the rise

otime

onumber of cells of the shift register $S R$ is equal to $N$.

8Generated PRBS is stored in the Output Register PRB.

fIt has a binary form, i.e., it assumes either the value of ta or $-a$.

oIf $T s$ is the sample period, then Tprbs $=p^{\star} T s$ with $p$ being an integer, sout it is recommended to choose $p<=4$.

clf

$p=i n p u t$ ('p (choose so that NTsp>=tr) =');

$\mathrm{N}=$ input $(' \mathrm{~N} \quad(\sim=t r / T p r b s=t r / p * T s<=4-10)=') ;$ ouser-defined parameter

a=input ('a (magnitude) $=$ ') ; ouser-defined parameter

$L=2 \wedge N-1 ;$ iflength of the sequence stored in the output register PRB for $p=1$

$L L=p * I$;

$\mathrm{PRB}=$ zeros (1,LL); finitialization of the output Register

$S R=o n e s(1, N) ; \quad$ rinitialization of the Shift Register

SR1=zeros $(1, N)$; fod 2 sum of the first and last digit of $S R$ is ostored in SR1 (1)

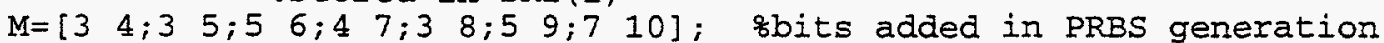

$I I=M(N-3,1) ; I h=M(N-3,2) ;$ zdetermination of the positions

$\mathrm{n}=1$; sof SR added modulo 2

for $m=1: I$

$\operatorname{SRSUM}=S R(I i)+\operatorname{SR}(1 \mathrm{~h})$;

\&SUMMATION MODULO 2

if SRSUM $==0$

$\operatorname{SRI}(1)=0$;

elseif SRSUM==2

else

$\operatorname{SRI}(1)=0$;

end

$\operatorname{SR} 1(1)=1 ;$

\&OUTPUT GENERATION

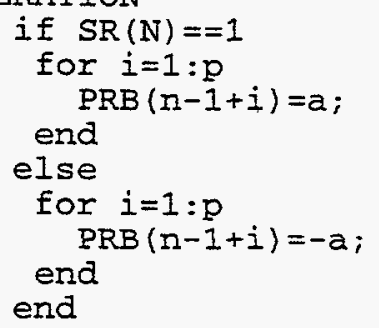

$n=n+p$; 
\&SHIFTING SR TO THE RIGHT AND UPDATING

for $1=1: N-1$

$\mathrm{SR}(\mathrm{N}+1-1)=\mathrm{SR}(\mathrm{N}-1)$;

$\operatorname{SR}(1)=0$;

end

$S R=S R+S R 1$

8

$k=0: L L-1$;

[kk, PP] =stairs $(k, P R B)$;

$\mathrm{v}=[0, \mathrm{LL},-1.2 * a,-1.2 * a]$;

plot (kk, PP) , ...

$\operatorname{axis}(v), \ldots$

title('PRBS Test Signal'),...

xlabel ('Time, samples'),...

ylabel ('Magni tude'); 


\section{Identification Programs}

There are three programs programs used in this research for identification of the wind turbine tower structure:

- fiokuy.m which is a modification of the program, with the same name, developed in the previous research and based on the observer/Kalman filter identification algorithm;

- idss2tfr.m which, for a selected input/output pair, converts the state-space model, generated by fiokuy.m, to the transfer function model and displays the corresponding frequency response;

- zoomtfr.m which allows the user to zoom on a selected part of both magnitude and phase frequency response, generated by idss 2 tfr.m.

System/Observer/Controller Identification Toolbox [3], developed at NASA Langley Research Center, is required to run these programs. The user of fiokuy.m, with the help of graphical and numerical information, generated by the program, selects the desired model order for which modal parameters are then displayed and the observer/Kalman filter (state-space) model is generated. The identification is based on a frequency-rich input/output history. The program idss 2 tfr.m displays identified system eigenvalues and corresponding modal frequencies in $\mathrm{rad} / \mathrm{s}$ and $\mathrm{Hz}$, as well as system zeros related to the selected output. The application notes and test results are presented in Appendix B.

The information on user-defined parameters and variables available to the user is included in the Matlab responses to help fiokuy, help idss2tfr, and help zoomtfr, as shown below. The listings of all three programs immediately follow.

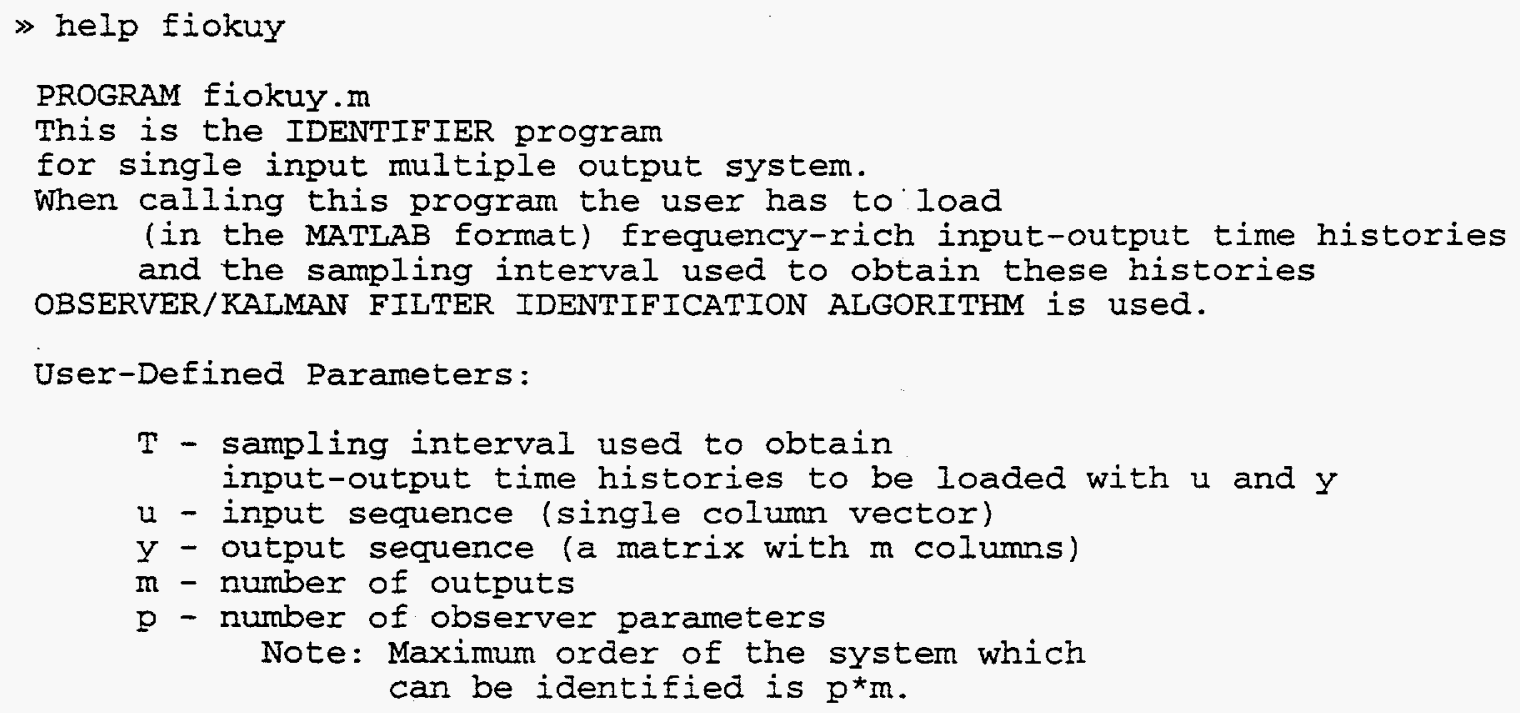


Variables Available:

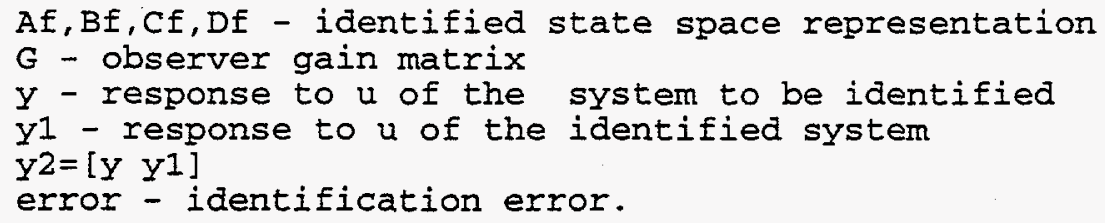

》help idss2tfr.

PROGRAM idss2tfr.m

This program uses identified state space representation, obtained from input-output data set using fiokuy.m, and generates eigenvalues of the identified system, available as a vector $r 1$. Each row of the generated matrix numl represents numerator coefficients of a transfer function associated with this row. It is assumed that the number of the output is identical with the row number of numl. The vector den1 represents the denominator polynomial coefficients. For the output number, declared by the user, the system plots frequency response. The axis (frequency, magnitude, and phase ranges) are scaled automatically. To zoom on a particular part of the frequency response, run zoomtfr.m.

$\gg$ help zoomtfr

PROGRAM zoomtfr.m

This program uses the model generated by idss 2 tfr.m.

For the output number, declared by the user,

the system plots frequency response on a linear scale and within the requested frequency range.

The user is asked to declare axis (frequency, magnitude, and phase ranges) and number of points within the selected frequency range.

》 type fiokuy

\&PROGRAM fiokuY.m

othis is the IDENTIFIER program

ffor single input multiple output system.

owhen calling this program the user has to load

\% (in the MATLAB format) frequency-rich input-output time histories

\& and the sampling interval used to obtain these histories \&OBSERVER/KALMAN FILTER IDENTIFICATION ALGORITHM is used. 


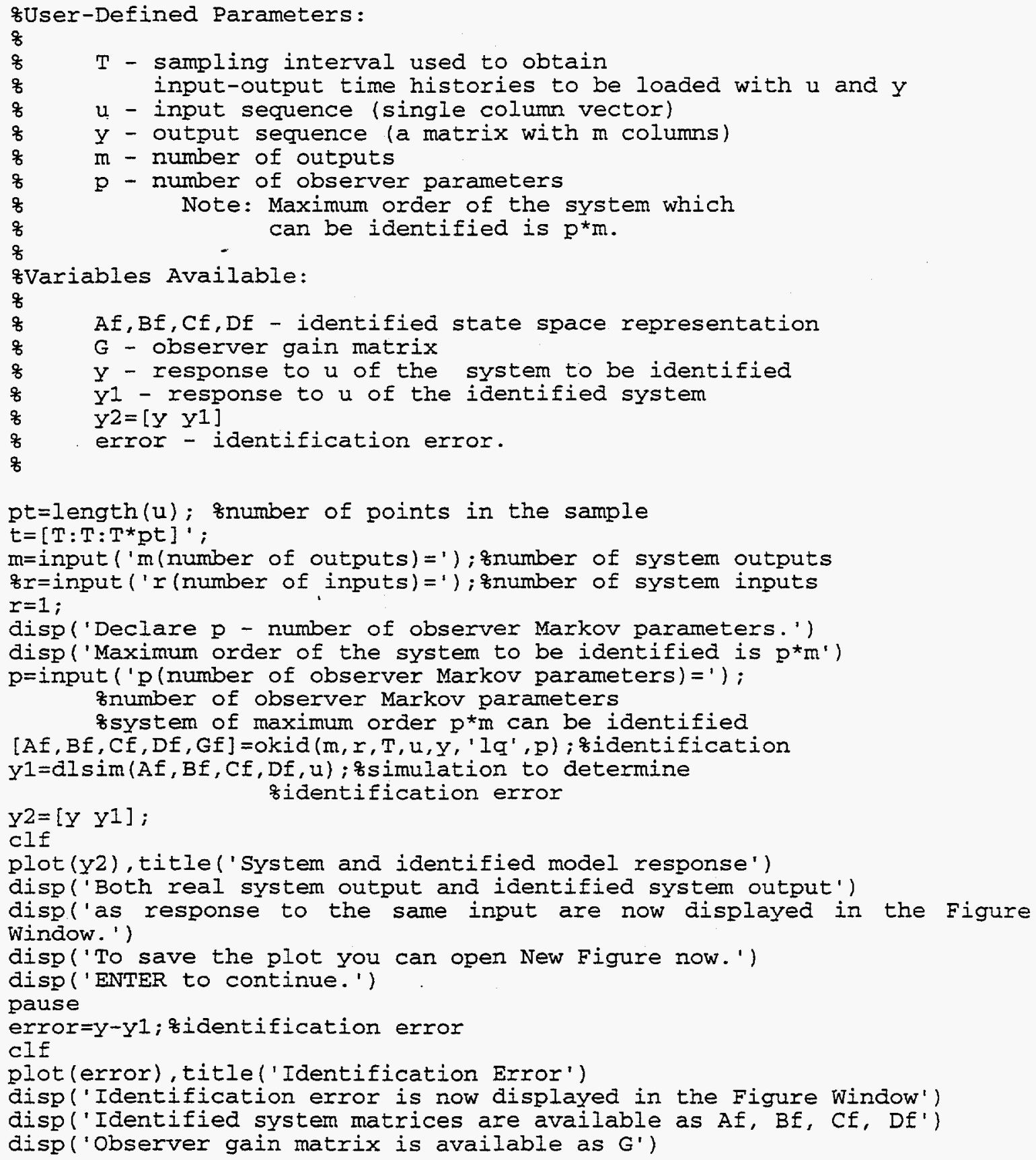


\%PROGRAM idss2tfr.m

othis program uses identified state space representation,

sobtained from input-output data set using fiokuy.m,

sand generates eigenvalues of the identified system,

ravailable as a vector $\mathrm{rl}$. Each row of the generated matrix

snuml represents numerator coefficients of a transfer

gfunction associated with this row. It is assumed that the

snumber of the output is identical with the row number of numl.

othe vector derl represents the denominator polynomial coefficients.

\&For the output number, declared by the user,

othe system plots frequency response.

othe axis (Erequency, magnitude, and phase ranges) are scaled

zautomatically. To zoom on a particular part of the frequency

oresponse, run zoomtfr.m.

onr=input ('onr (output $n r$ for freq. resp) =');

$[a 1, \mathrm{~b} 1]=\mathrm{d} 2 \mathrm{C}(\mathrm{Af}, \mathrm{Bf}, \mathrm{T})$; \&identified continuous system is obtained.

[numl, denl] =ss2tf $(a 1, b 1, C f, D f, 1)$; 8 identified transfer function

disp ('IDENTIFIED SYSTEM EIGENVALUES')

rl=roots $(\operatorname{den} 1)$ \&identified eigenvalues

wn=abs ( $r 1)$; 8 identified mode frequencies in rad/s

fn=wn/ $(2 * \mathrm{pi}) ; 8$ identified mode frequencies in $\mathrm{Hz}$

$\mathrm{fr}=[\mathrm{wm} \mathrm{fn}]$;

disp('IDENTIFIED MODAL FREQUENCIES IN rad/s AND Hz')

disp(' $\mathrm{rad} / \mathrm{s} \mathrm{Hz}$ ')

Er

Eprintf('IDENTIFIED SYSTEM ZEROS RELATED TO THE OUTPUT \&g\n', ONY)

ZER=roots (numl (onr, :)) \&system zeros

$z w n=a b s(Z E R) ; 8$ identified zero frequencies in $\mathrm{rad} / \mathrm{s}$

$z$ fn $=z W n /(2 *$ pi $)$; qidentified zero frequencies in $\mathrm{Hz}$

$z f r=[z w n z f n]$;

disp ('IDENTIFIED ZERO FREQUENCIES IN rad/s AND $\mathrm{Hz}$ ')

disp(' $\mathrm{rad} / \mathrm{s} \mathrm{Hz}$ ')

$z$ fr

[mag1, phase1, w] =dbode (Af, Bf,Cf,Df, T, 1); fmagl and phasel have

znumber. of columns equal to the number of outputs

$\operatorname{lmag} 1=20 * \log (\operatorname{mag} 1($ : , onr $))$;

subplot $(2,1,1)$

semilogx (w, Imag1, 'r'), grid, ...

xlabel ('Frequency (rad/sec)'),...

ylabel ('Gain $\left.d B^{\prime}\right), \ldots$

title (['FREQUENCY RESPONSE FOR OUTPUT ', num2str(onr)]),...

subplot $(2,1,2)$

semilogx (w, phase1 (: , onr $),$ 'r'), grid, ...

xlabel ('Frequency ( $\mathrm{rad} / \mathrm{sec}$ )'),...

Ylabel ('Phase deg')

fprintf ('Frequency response for OUTPUT $8 \mathrm{~g}$ is now displayed $\backslash \mathrm{n}$ ', onx)

disp('in the Figure Window.')

disp('If you want to obtain frequency response for more than one output, ')

disp('run idss2tfr.m again and/or run zoomtfr.m to look')

disp('at a specified frequency range.') 
\%PROGRAM zOOmtfr.m

FThis program uses the model generated by idss2tfr.m.

\&For the output number, declared by the user,

othe system plots frequency response on a linear scale and

owithin the requested irequency range.

the user is asked to declare axis (frequency, magnitude,

fand phase ranges) and number of points within the selected

\&frequency range.

onr=input ('onr (-output nr for freq. resp) =');

ml=input ('ml (gain magnitude low Iimit in $\mathrm{dB}$ ) =') ;

mh=input ('mh (gain magnitude high limit in $d B$ ) =') ;

fl=input (' $f l$ (freq. range low limit in $\mathrm{rad} / \mathrm{s}$ ) =');

fh=input ("Eh (freq. range high limit in $\mathrm{rad} / \mathrm{s}$ ) $=$ ");

phl=input ('phl (phase low limit in deg)=');

phh=input ('phh (phase high limit in deg) =');

np=input ('np (number of points between $f l$ and fh) =');

$w=l$ inspace $(\mathrm{fl}, \mathrm{fh}, \mathrm{np})$;

$[\operatorname{mag} 1$, phasel, $w]=d b o d e(A f, B f, C f, D f, T, 1, W) ;$ magl and phasel have

snumber of columns equal to the number of outputs

$\operatorname{lmag} 1=20 * \log (\operatorname{mag} 1(:$, onr $))$;

$\mathrm{v}=[\mathrm{fl}, \mathrm{fh}, \mathrm{ml}, \mathrm{mh}]$;

subplot $(2,1,1)$

plot (w, Imagl, 'r'),grid, ...

axis $(v), \ldots$

xlabel ('Frequency (rad/sec)'),...

ylabel ('Gain $\left.d B^{\prime}\right), \ldots$

title(['FREQUENCY RESPONSE FOR OUTPUT ', num2str(onr)]),...

subplot $(2,1,2)$

plot (w, phasel (:,onr), 'r'), grid, ...

$v 1=[f l, f h, p h l, p h h] ;, \ldots$

$\operatorname{axis}(v 1), \ldots$.

xlabel ('Frequency (rad/sec)'), ...

ylabel ('Phase deg')

disp(')

fprintf('Frequency response for ouTPUT ofg is now displayed $\backslash n$ ', onr)

disp("in the Figure Window. ")

disp("')

disp('If you want to obtain zoomed frequency response for more than one output, ')

disp('run zoomtfr.m again or idss2tfr for full frequency response') 


\section{APPENDIX B}

\section{Application Notes and Identification Results Obtained Using ADAMS Simulation Data for the Wind Turbine Micon 65/13}

The purpose of this appendix is twofold. It can be viewed as a practical step-by-step guide to system identification, illustrated by a complete example, with special emphasis on identification of modal parameters. On the other hand, it constitutes a detailed outline of an approach to validation of the ADAMS model with experimental modal test data when such become available. In this case, the data processing, performed here for the measurement data generated by the simulation, has to be repeated using experimental data. The modal parameters obtained can then be compared for validation.

As outlined in Section 2.2, the excitation of all modes requires obtaining input-output time-series for both in-plane and out-of-plane excitations applied at various points of a wind turbine structure. For out-of-plane excitations, collocated with the measurements TOWER4_Z, B2S13_Y, and B1S13_Y, five measurements listed in Table 2.3 have been selected and the corresponding measurement data files itf0104r.dat, ib20104r.dat, and ib10104r.dat have been formed. In addition, one in-plane excitation, collocated with the measurement TOWER4_Y, has been applied. The measurements included in the reduced data file its0104r.dat, formed for this excitation, are listed in Table 2.4. The measurements included in all these data files are referred in this appendix as outputs with a proper number assigned as shown in Table 2.3 and Table 2.4 .

For all of these data files, the same identification procedure is repeated. First, after loading a particular data file into MATLAB work area, the program fiokuy.m is executed. The user is asked for the number of outputs (or number of measurements) and the number of Markov parameters to be considered. The user, then considering the plot of singular values makes the decision as to what should be the order of the system model to be generated. The program identifies a state-space representation of a system (in the form of an observer/Kalman filter model) and returns the identified system's modal frequencies and damping values with the corresponding modal amplitude coherence (MAC) factors and mode singular values. Even though the details how the user can distinguish noise modes from real modes based on these data are outlined in Section 3.1 as well as in [1], the following summary information is in place. Modes to be considered as real modes must have MAC value of 1 or close to 1 meaning that the response history associated with a particular mode of the identified model and the measured response history (taken as vectors) coincide. In addition, modes with small singular values can be neglected because they do not contribute much to the structure's dynamics. In what follows, the modal frequencies to be considered as representing real modes are highlighted in bold. The identification error is displayed in the Figure Window. 
Then, the identification programs idss2tfr.m and zoomtfr.m are usually repeated the number of times equal to the number of measurement points in the processed data file (five times in the identification process presented below). This is justified by the fact that for each input-output relation in a given file, we have the same poles or modal frequencies but different zeros. Therefore, each input-output pair has a different frequency response. As we can see from the identification results presented below, the visibility of the modal frequencies on the frequency response plots is different for different input-output pairs and, of course, much sharper for those pairs that are collocated. This is justified by the signal-to-noise factor which is much more faveorable for a response collocated with the excitation. Also, we can observe that for the excitation inputs applied at any point on the turbine axis of symmetry (files ib10104r.dat and itf0104r.dat), the frequency responses for symmetrical outputs are identical. Considering the possible wide range of research avenues which can be pursued using this software, the idss 2 tfr.m program returns the following list of the identified system parameters: system eigenvalues, modal frequencies in [rad/s] and [Hz], system zeros and their frequencies in $[\mathrm{rad} / \mathrm{s}]$ and $[\mathrm{Hz}]$. The application of the program zoomtfr.m is self-explanatory.

Each of the measurement files generated in the simulation contains data for 64 different measurement points. Therefore, many different data files can be formed to investigate input-output relations of interest using the same set of the developed MATLAB programs. The identification procedure and the presentation of results for four data files defined above follows.

Identification Procedure and Results for the Data File itf0104r.dat

\section{Loading the Data File}

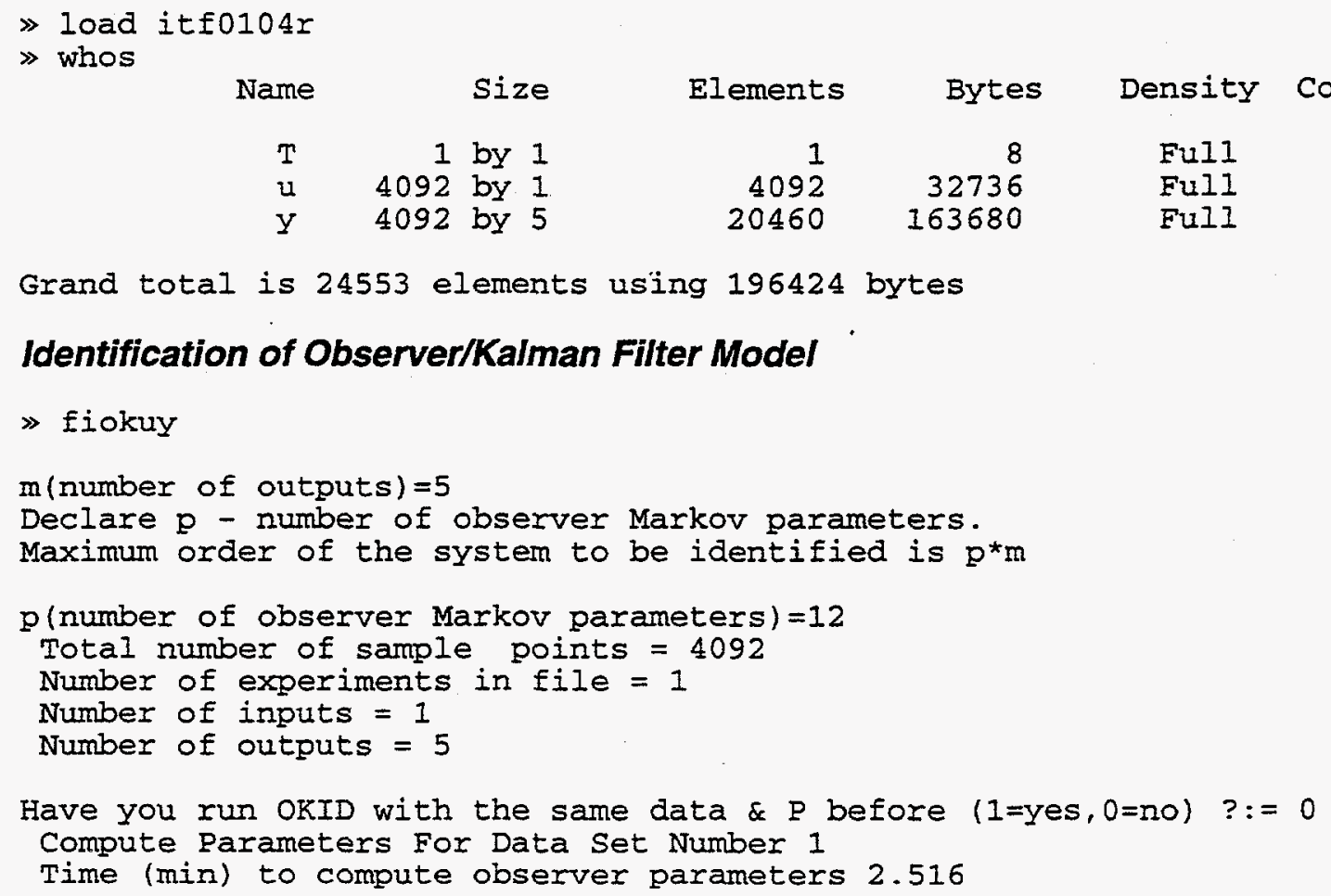

\section{Identification of Observer/Kalman Filter Model}

》fiokuy

$\mathrm{m}$ (number of outputs) $=5$

Declare p - number of observer Markov parameters.

Maximum order of the systern to be identified is $p^{*} m$

$p$ (number of observer Markov parameters) $=12$

Total number of sample points $=4092$

Number of experiments in file $=1$

Number of inputs $=1$

Number of outputs $=5$

Have you run OKID with the same data \& $P$ before $(1=y e s, 0=$ no $) ~ ?:=0$

Compute Parameters For Data set Number 1

Time (min) to compute observer parameters 2.516 
Compute Prediction Error ( 1 =yes, $0=$ no) ? =: 0

Time (min) to compute Markov parameters 0.2142

ERADC is used now.

The Hankel matrix size for ERADC is 60 by 181 .

Maximum Hankel singular value $=2.175528 \mathrm{e}-003$

Minimum Hankel singular value $=2.099507 \mathrm{e}-021$

Desired Model arder $(0=$ stop $)=: 34$

Model Describes $100(\%)$ of Test Data

Damping ( 8 ) Freq(HZ) Mode SV

$1.0000 e+002 \quad 2.4774 e+001 \quad 2.4688 e-001$

$1.8502 e+001 \quad 5.0878 e+001 \quad 2.4718 e-001$

$7.0422 e+000 \quad 4.1346 e+001 \quad 2.3116 e-001$

$7.0422 \mathrm{e}+000 \quad 4.1346 \mathrm{e}+001 \quad 2.3116 \mathrm{e}-001$

$4.8947 e+000 \quad 4.3395 e+001 \quad 4.9625 e-001$

$4.89 .47 e+000 \quad 4.3395 e+001 \quad 4.9625 e-001$

$1.7576 e+001 \quad 1.1780 e+001 \quad 2.8978 e-001$

$1.7576 \mathrm{e}+001 \quad 1.1780 \mathrm{e}+001 \quad 2.8978 \mathrm{e}-001$

$1.0814 e+001$

$1.8163 e+001$

$1.0814 \mathrm{e}+001$

$1.8163 e+001$

$5.5617 e+000$

$3.2834 e+001$

$5.5617 e+000$

$3.2834 e+001$

$2.4137 e+001$

$7.1055 e+000$

$2.4137 e+001$

$7.1055 e+000$

$3.8738 e-001$

$3.8738 e-001$

$3.2798 e-001$

$3.2798 e-001$

$2.3707 e-001$

$2.3707 e-001$

$5.5384 e+000$

$2.9987 e+001$

$4.8412 e-001$

$2.9987 e+001$

$4.8412 e-001$

$5.5384 e+000$

$3.4799 e+001$

3. $6827 e-001$

$4.4314 e+000$

$3.4799 e+001$

$3.6827 e-001$

$5.9353 e+000$

$2.0354 e+001$

$4.8383 e-001$

$5.9353 e+000$

$2.0354 e+001$

$4.8383 e-001$

$5.8685 e+000$

$1.5871 e+001$

$4.8823 e-001$

$5.8685 e+000$

$1.5871 \mathrm{e}+001$

$4.8823 e-001$

$7.0006 e+000$

$1.2338 e+001$

$4.4115 e-001$

$1.2338 \mathrm{e}+001$

$4.4115 e-001$

$7.0006 e+000$

$1.0824 e+001$

$5.3713 e+000$

$1.0824 e+001$

$3.8899 e-001$

$3.8899 e-001$

$6.2668 e+000$

$8.6730 e+000$

$3.6710 e-001$

$8.6730 e+000$

3. $6710 e-001$

$5.9811 e+000$

$3.5064 e+000$

$9.9079 e-001$

$5.9811 e+000$

$3.5064 e+000$

$9.9079 e-001$

$1.7626 e+000$

$7.8050 e-001$

$9.6484 e+000$

$1.7626 e+000$

$7.8050 e-001$

$2.5184 e+000$

$3.1968 e+000$

$1.0000 e+000$

$3.1968 e+000$

1. $0000 e+000$

MAC

$9.9763 e-001$

$9.9838 e-001$

$9.9937 e-001$

$9.9937 e-001$

$9.9998 e-001$

$9.9998 e-001$

$9.9923 e-001$

$9.9923 e-001$

$9.9996 e-001$

$9.9996 e-001$

$9.9987 e-001$

$9.9987 e-001$

$9.9977 e-001$

$9.9977 e-001$

$9.9999 e-001$

$9.9999 e-001$

$9.9996 e-001$

$9.9996 e-001$

$1.0000 e+000$

$1.0000 e+000$

$1.0000 e+000$

$1.0000 e+000$

$9.9998 \mathrm{e}-001$

$9.9998 \mathrm{e}-001$

$9.9999 e-001$

$9.9999 e-001$

$9.9999 e-001$

$9.9999 e-001$

$1.0000 e+000$

$1.0000 e+000$

$1.0000 e+000$

$1.0000 e+000$

$1.0000 e+000$

$1.0000 e+000$

Desired Model order $(0=s t o p)=: 0$

Compare Recons. Output and True output $(1=y e s, 0=$ no) $?:=0$

Both real system output and identified system output

as response to the same input are now displayed in the Figure window.

To save the plot you can open New Figure now.

ENTER to continue.

Identification error is now displayed in the Figure Window Identified system matrices are available as Af, Bf, Cf, Df observer gain matrix is available as $G$

$\gg$ 

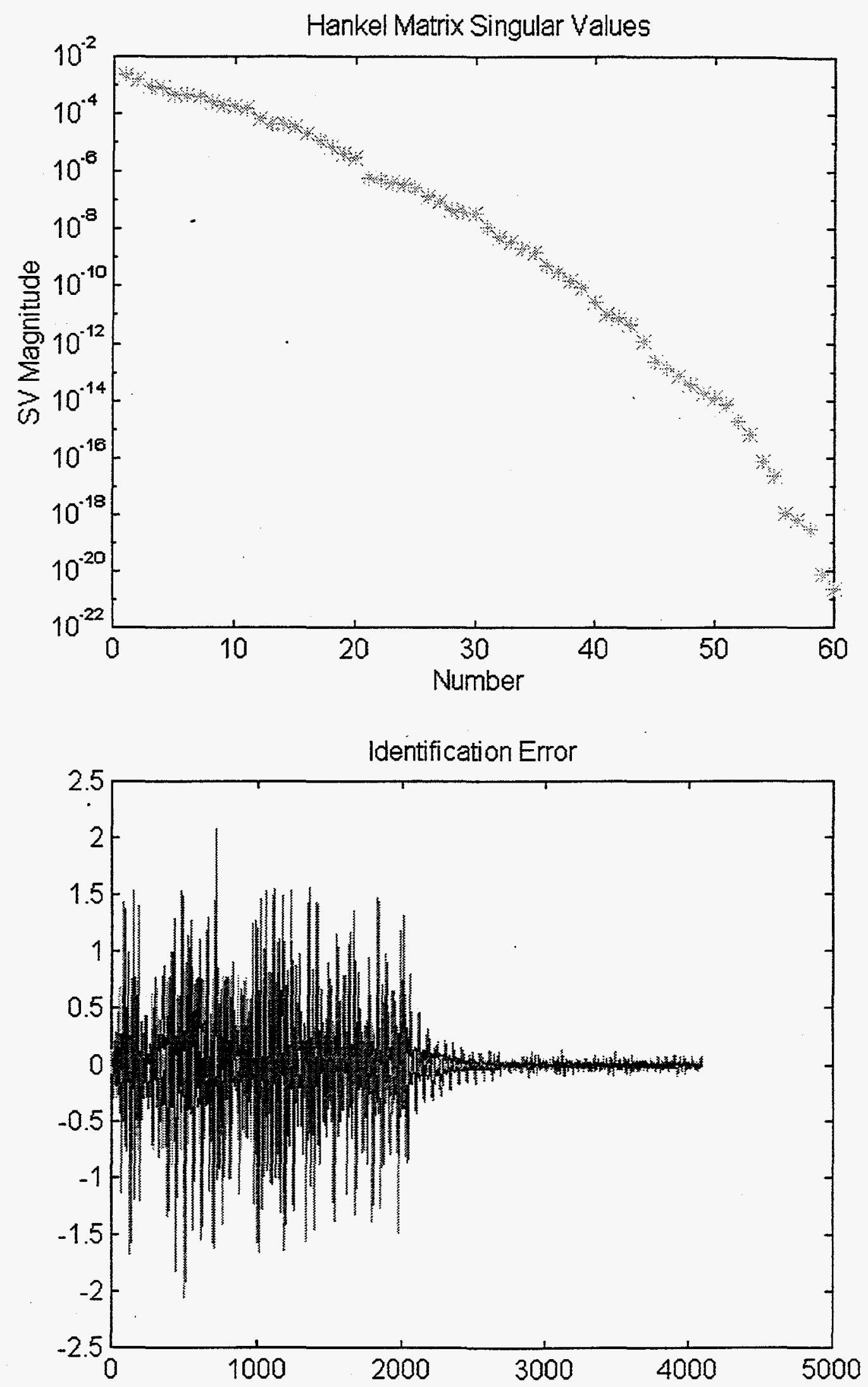


\section{Frequency Response for OUTPUT1 (Collocation Case)}

$\gg$ idss2tfr

onr (output nr for freq. resp) $=1$

IDENTIFIED SYSTEM EIGENVALUES

$r 1=$

$-1.3346 e+001+2.7233 e+002 i$

$-1.3346 e+001-2.7233 e+002 i$

$-1.8295 e+001+2.5914 e+002 i$

$-1.8295 e+001-2.5914 e+002 i$

$-9.6893 e+000+2.1843 e+002 i$

$-9.6893 e+000-2.1843 e+002 i$

$-1.1474 e+001+2.0598 e+002 i$

$-1.1474 e+001-2.0598 e+002 i$

$-1.0435 e+001+1.8812 e+002 i$

$-1.0435 e+001-1.8812 e+002 i$

$-1.5566 e+002$

$-7.5905 e+000+1.2766 e+002 i$

$-7.5905 e+000-1.2766 e+002 i$

$-1.2341 e+001 \div 1.1345 e+002 i$

$-1.2341 e+001-1.1345 e+002 i$

$-5.8522 e+000+9.9550 e+001 i$

$-5.8522 e+000-9.9550 e+001 i$

$-5.9147 e+001$

$-5.4268 e+000+7.7330 e+001 i$

$-5.4268 e+000-7.7330 e+001 i$

$-1.3009 e+001+7.2863 e+001 i$

$-1.3009 e+001-7.2863 e+001 i$

$-3.6530 e+000+6.7912 e+001 i$

$-3.6530 e+000-6.7912 e+001 i$

$-3.4150 e+000+5.4387 e+001 i$

$-3.4150 e+000-5.4387 e+001 i$

$-1.0776 e+001+4.3325 e+001 i$

$-1.0776 e+001-4.3325 e+001 i$

$-1.3177 e+000+2.1992 e+001 i$

$-1.3177 e+000-2.1992 e+001 i$

$-5.0585 e-001+2.0080 e+001 i$

$-5.0585 e-001-2.0080 e+001 i$

$-1.0685 e+000+1.1023 e+001 i$

$-1.0685 e+000-1.1023 e+001 i$

IDENTIFIED MODAL FREQUENCIES IN $\mathrm{rad} / \mathrm{s}$ AND $\mathrm{Hz}$ fr $=$

$$
\mathrm{rad} / \mathrm{s} \quad \mathrm{Hz}
$$
$2.7266 e+002$
$2.7266 e+002$
$4.3395 e+001$
$2.5978 \mathrm{e}+002$
$2.5978 e+002$
$4.3395 e+001$
$4.1346 e+001$
$2.1865 e+002$
$4.1346 e+001$
$2.1865 e+002$
$3.4799 e+001$
$3.4799 e+001$
$2.0630 e+002$
$2.0630 e+002$
$3.2834 e+001$
$1.8841 e+002$
$3.2834 e+001$
$1.8841 \mathrm{e}+002$
$2.9987 e+001$
$1.5566 \mathrm{e}+002$
$2.9987 e+001$
$2.4774 \mathrm{e}+001$
$1.2789 e+002$
$1.2789 e+002$
$1.1412 \mathrm{e}+002$
$2.0354 e+001$
$2.0354 e+001$
$1.8163 e+001$
$1.1412 \mathrm{e}+002$
$9.9722 \mathrm{e}+001$
$1.8163 e+001$
$1.5871 e+001$
$9.9722 e+001$
$5.9147 e+001$
$1.5871 e+001$
$7.7520 e+001$
$9.4135 e+000$
$7.7520 e+001$
$1.2338 e+001$
$1.2338 e+001$ 
$7.4015 e+001$

$7.4015 e+001$

$6.8010 e+001$

$6.8010 e+001$

$5.4494 e+001$

$5.4494 e+001$

$4.4645 e+001$

$4.4645 e+001$

$2.2031 e+001$

$2.2031 e+001$

$2.0086 e+001$

$2.0086 e+001$

$1.1075 e+001$

$1.1075 \mathrm{e}+001$
$1.1780 \mathrm{e}+001$

$1.1780 \mathrm{e}+001$

$1.0824 \mathrm{e}+001$

$1.0824 e+001$

$8.6730 e+000$

$8.6730 \mathrm{e}+000$

$7.1055 \mathrm{e}+000$

$7.1055 e+000$

$3.5064 e+000$

$3.5064 \mathrm{e}+000$

$3.1968 \mathrm{e}+000$

$3.1968 \mathrm{e}+000$

$1.7626 e+000$

$1.7626 e+000$

IDENTIFIED SYSTEM ZEROS RELATED TO THE OUTPUT 1 $\mathrm{ZER}=$

$3.1139 e+002+3.6853 e+002 i$

$3.1139 e+002-3.6853 e+002 i$

$-2.1704 e+001+2.5902 e+002 i$

$-2.1704 e+001-2.5902 e+002 i$

$-1.0073 e+001+2.3527 e+002 i$

$-1.0073 e+001-2.3527 e+002 i$

$-1.5076 e+001+2.1052 e+002 i$

$-1.5076 e+001-2.1052 e+002 i$

$-2.4163 e+001+1.9487 e+002 i$

$-2.4163 e+001-1.9487 e+002 i$

$-1.9364 \mathrm{e}+001+1.3254 \mathrm{e}+002 i$

$-1.9364 e+001-1.3254 e+002 i$

$-4.8435 e+000+1.0962 e+002 i$

$-4.8435 e+000-1.0962 e+002 i$

$3.3462 e+001+9.5703 e+001 i$

$3.3462 e+001-9.5703 e+001 i$

$-8.0594 e+001$

$-9.1650 e+000+8.4268 e+001 i$

$-9.1650 e+000-8.4268 e+001 i$

$-4.3114 e+000+7.0832 e+001 i$

$-4.3114 \dot{e}+000-7.0832 e+001 i$

$-8.1020 e+000+6.5483 e+001 i$

$-8.1020 e+000-6.5483 e+001 i$

$-1.7053 e+001+4.8927 e+001 i$

$-1.7053 e+001-4.8927 e+001 i$

$-3.4549 e+000+5.0486 e+001 i$

$-3.4549 e+000-5.0486 e+001 i$

$-9.2851 e+000+1.6901 e+001 i$

$-9.2851 e+000-1.6901 e+001 i$

$-3.1281 e+000+2.0829 e+001 i$

$-3.1281 e+000-2.0829 e+001 i$

$-3.7882 e-001+1.9711 e+001 i$

$-3.7882 e-001-1.9711 e+001 i$

$4.0870 e+000$

IDENTIFIED ZERO FREQUENCIES IN rad/s AND Hz $z$ fr $=$ $\mathrm{rad} / \mathrm{s} \quad \mathrm{Hz}$
$4.8247 e+002$
$4.8247 e+002$
$2.5992 e+002$
$2.5992 e+002$
$2.3548 \mathrm{e}+002$
$2.3548 e+002$
$2.1106 e+002$
$2.1106 e+002$
$1.9636 e+002$
$1.9636 e+002$
$1.3395 e+002$
$1.3395 e+002$
$7.6788 e+001$
$7.6788 e+001$
$4.1368 e+001$
$4.1368 e+001$
3. $7478 e+001$
$3.7478 e+001$
$3.3591 e+001$
$3.3591 e+001$
$3.1252 e+001$
$3.1252 \mathrm{e}+001$
$2.1318 e+001$
$2.1318 e+001$ 
1. $0972 \mathrm{e}+002$

$1.0972 \mathrm{e}+002$

1. $0138 \mathrm{e}+002$

$1.0138 e+002$

$8.0594 e+001$

$8.4765 e+001$

$8.4765 e+001$

$7.0963 e+001$

$7.0963 e+001$

$6.5982 \mathrm{e}+001$

$6.5982 \mathrm{e}+001$

$5.1813 e+001$

$5.1813 e+001$

$5.0604 e+001$

5. $0604 e+001$

1. $9283 e+001$

$1.9283 e+001$

$2.1063 e+001$

$2.1063 e+001$

$1.9715 e+001$

$1.9715 e+001$

$4.0870 e+000$
$1.7463 e+001$

$1.7463 e+001$

$1.6136 e+001$

$1.6136 e+001$

1. $2827 e+001$

$1.3491 e+001$

$1.3491 e+001$

$1.1294 \mathrm{e}+001$

1. $1294 \mathrm{e}+001$

1. $0501 e+001$

$1.0501 e+001$

$8.2464 e+000$

$8.2464 e+000$

$8.0539 e+000$

$8.0539 e+000$

$3.0690 e+000$

$3.0690 e+000$

3. $3522 \mathrm{e}+000$

$3.3522 \mathrm{e}+000$

3. $1377 \mathrm{e}+000$

$3.1377 e+000$

$6.5047 e-001$

Frequency response for OUTPUT 1 is now displayed in the Figure Window.

If you want to obtain frequency response for more than one output, run idss 2 tfr.m again and/or run zoomtfr.m to look at a specified frequency range.

$\gg$ 
$\gg$ zoomtfr

onr (output nr for freq. resp) $=1$

$\mathrm{ml}$ (gain magnitude low limit in $\mathrm{dB}$ ) $=-140$

mh(gain magnitude high limit in $\mathrm{dB}$ ) $=-60$

fl (freq. range low limit in rad/s) $=10$

fh (freq. range high limit in $\mathrm{rad} / \mathrm{s}$ ) $=300$

phl (phase low limit in deg) $=-800$

phh (phase high limit in deg) $=150$

$\mathrm{np}$ (number of points between $\mathrm{fl}$ and $\mathrm{fh}$ ) $=500$

Frequency response for OUTPUT 1 is now displayed

in the Figure Window.

If you want to obtain zoomed frequency response for more than one output,

run zoomtfr.m again or idss2tfr for full frequency response 

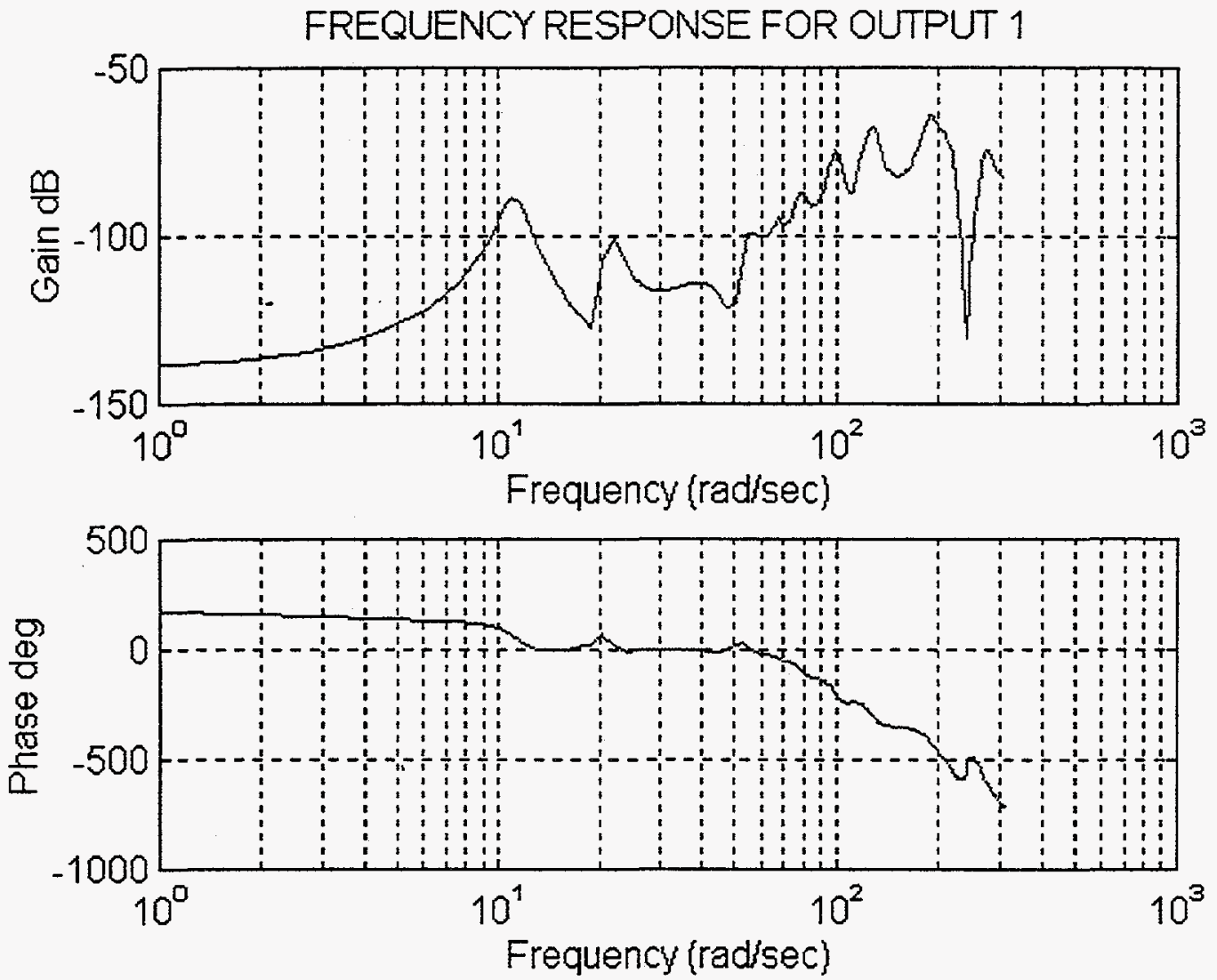

FREQUENCY RESPONSE FOR OUTPUT 1
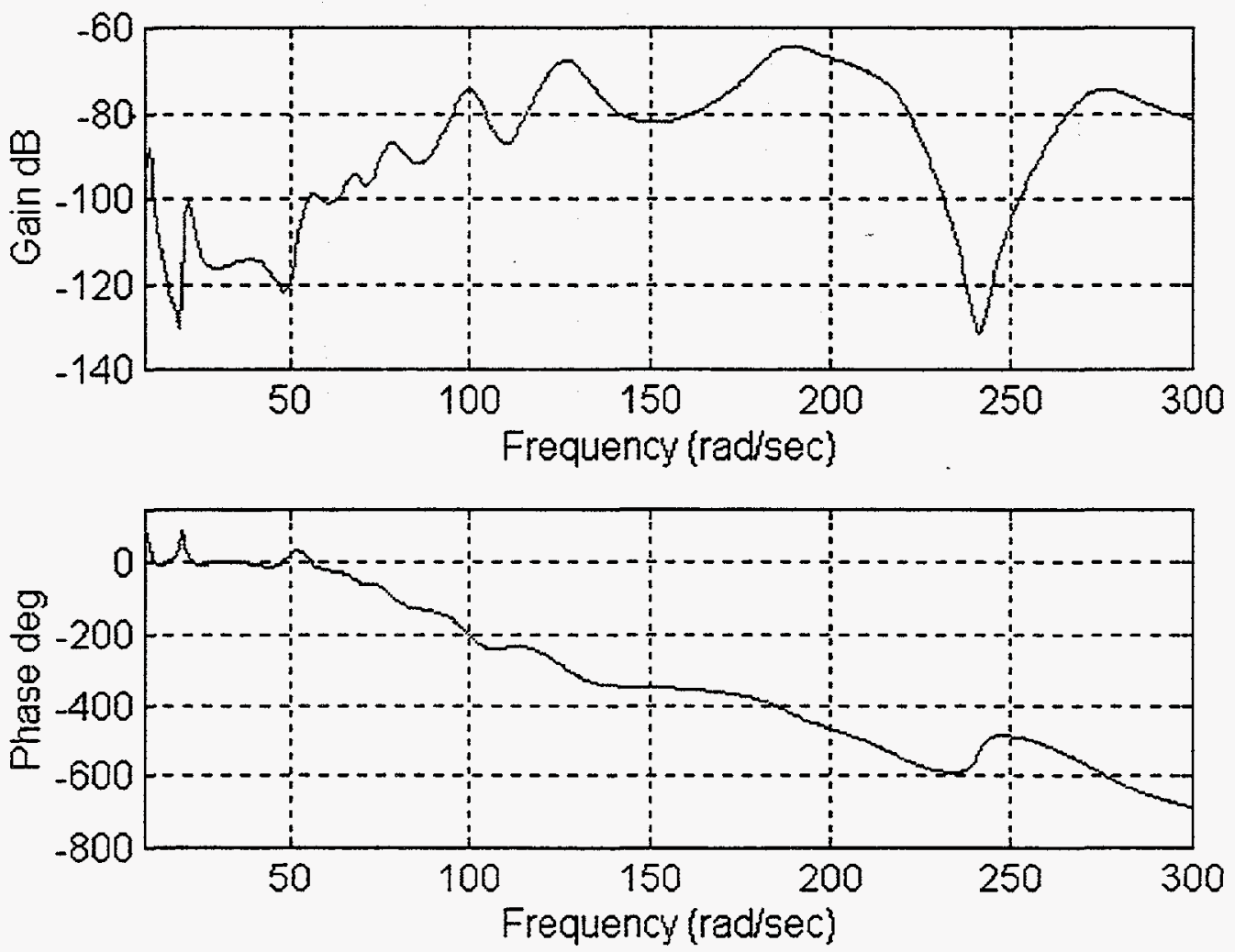


\title{
Frequency Response for OUTPUT2
}

\author{
$\gg$ idss2tfr
}

onr (output $\mathrm{nr}$ for freq. resp) $=2$

IDENTIFIED SYSTEM ZEROS RELATED TO THE OUTPUT 2 $\mathrm{ZER}=$

$-2.6174 \mathrm{e}+003$

$-1.2341 e+001+2.7388 e+002 i$

$-1.2341 e+001-2.7388 e+002 i$

$1.0418 e+001+2.2446 e+002 i$

$1.0418 e+001-2.2446 e+002 i$

$-1.6485 e+001+2.1824 e+002 i$

$-1.6485 e+001-2.1824 e+002 i$

$-8.2401 e+001+1.9214 e+002 i$

$-8.2401 e+001-1.9214 e+002 i$

$-1.0711 e+001+1.9806 e+002 i$

$-1.0711 e+001-1.9806 e+002 i$

$-1.8520 e+001+1.3659 e+002 i$

$-1.8520 e+001-1.3659 e+002 i$

$-8.4336 e+000+1.1433 e+002 i$

$-8.4336 e+000-1.1433 e+002 i$

$-1.4731 e+001+9.3537 e+001 i$

$-1.4731 e+001-9.3537 e+001 i$

$1.9232 e+001+7.6348 e+001 i$

$1.9232 e+001-7.6348 e+001 i$

$-5.3977 e+000+7.1150 e+001 i$

$-5.3977 e+000-7.1150 e+001 i$

$-9.6572 e+000+7.1180 e+001 i$

$-9.6572 e+000-7.1180 e+001 i$

$-1.5775 e+001+5.8270 e+001 i$

$-1.5775 e+001-5.8270 e+001 i$

$-7.9047 e+000+5.1083 e+001 i$

$-7.9047 e+000-5.1083 e+001 i$

$-4.3748 e+001$

$-2.9186 e+001$

$-9.4025 e-001+2.0152 e+001 i$

$-9.4025 e-001-2.0152 e+001 i$

$-1.0835 e+000+1.9301 e+001 i$

$-1.0835 e+000-1.9301 e+001 i$

$7.7912 e+000$ 


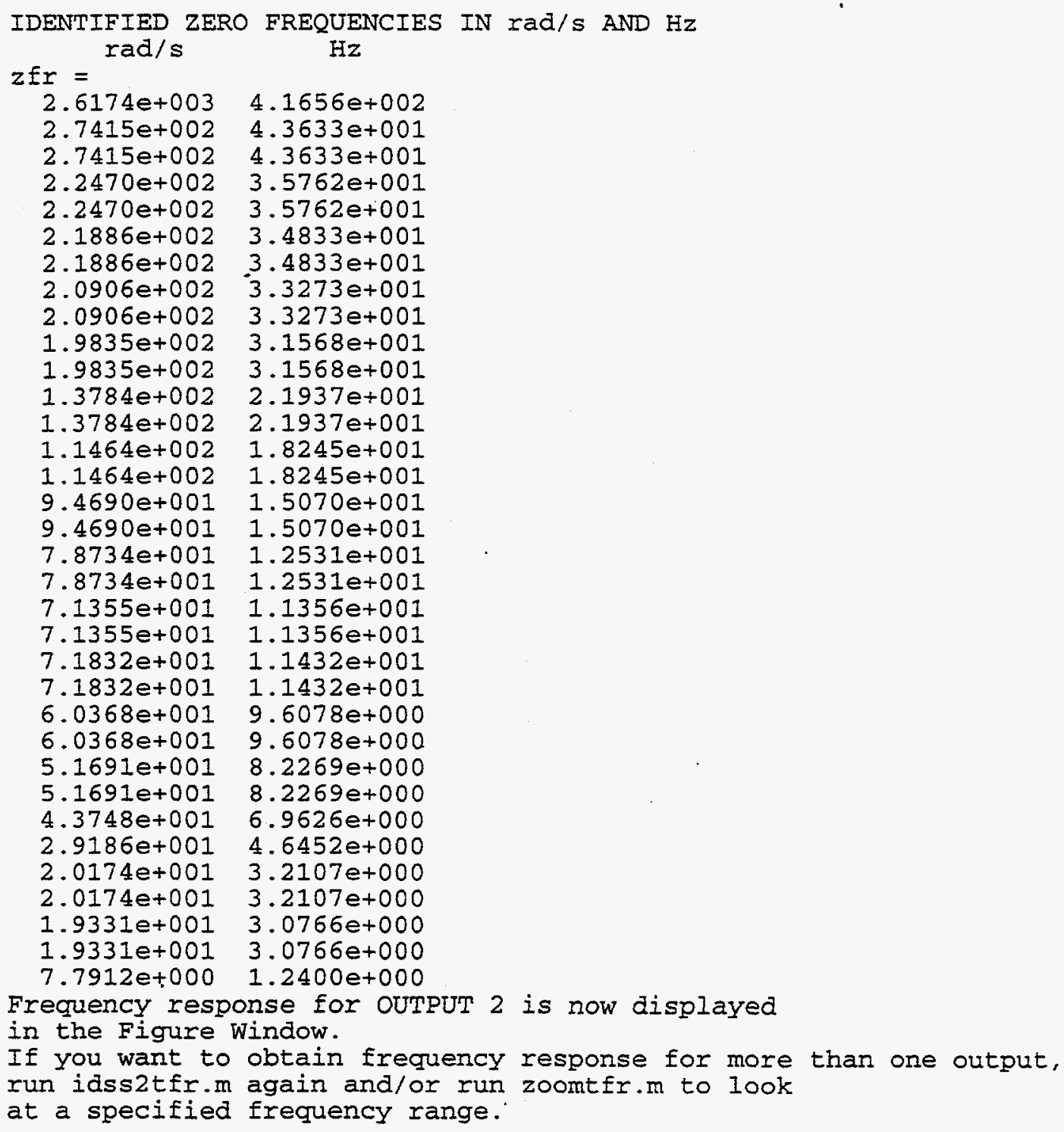



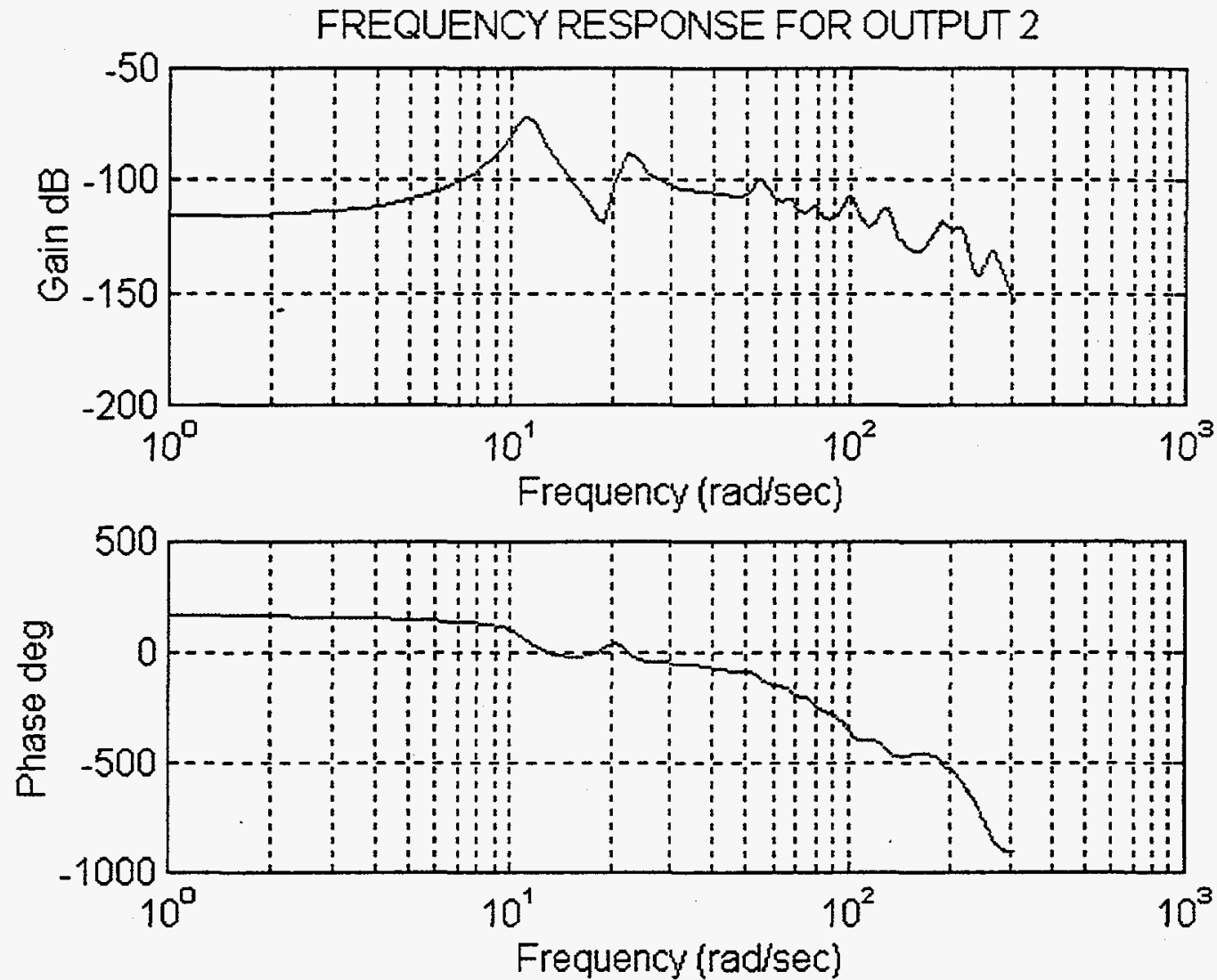

FREQUENCY RESPONSE FOR OUTPUT 2
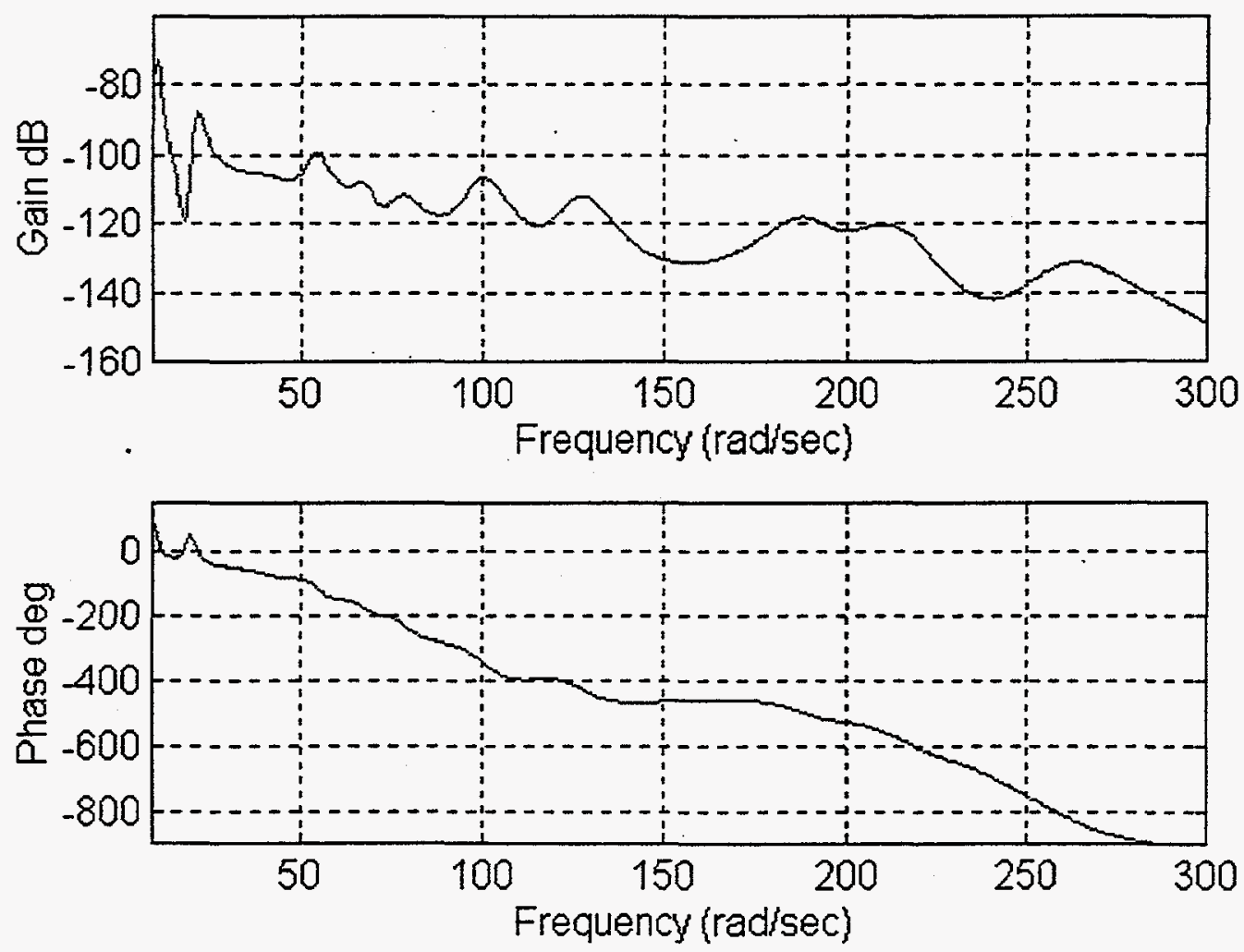


\section{Frequency Response for OUTPUT3}

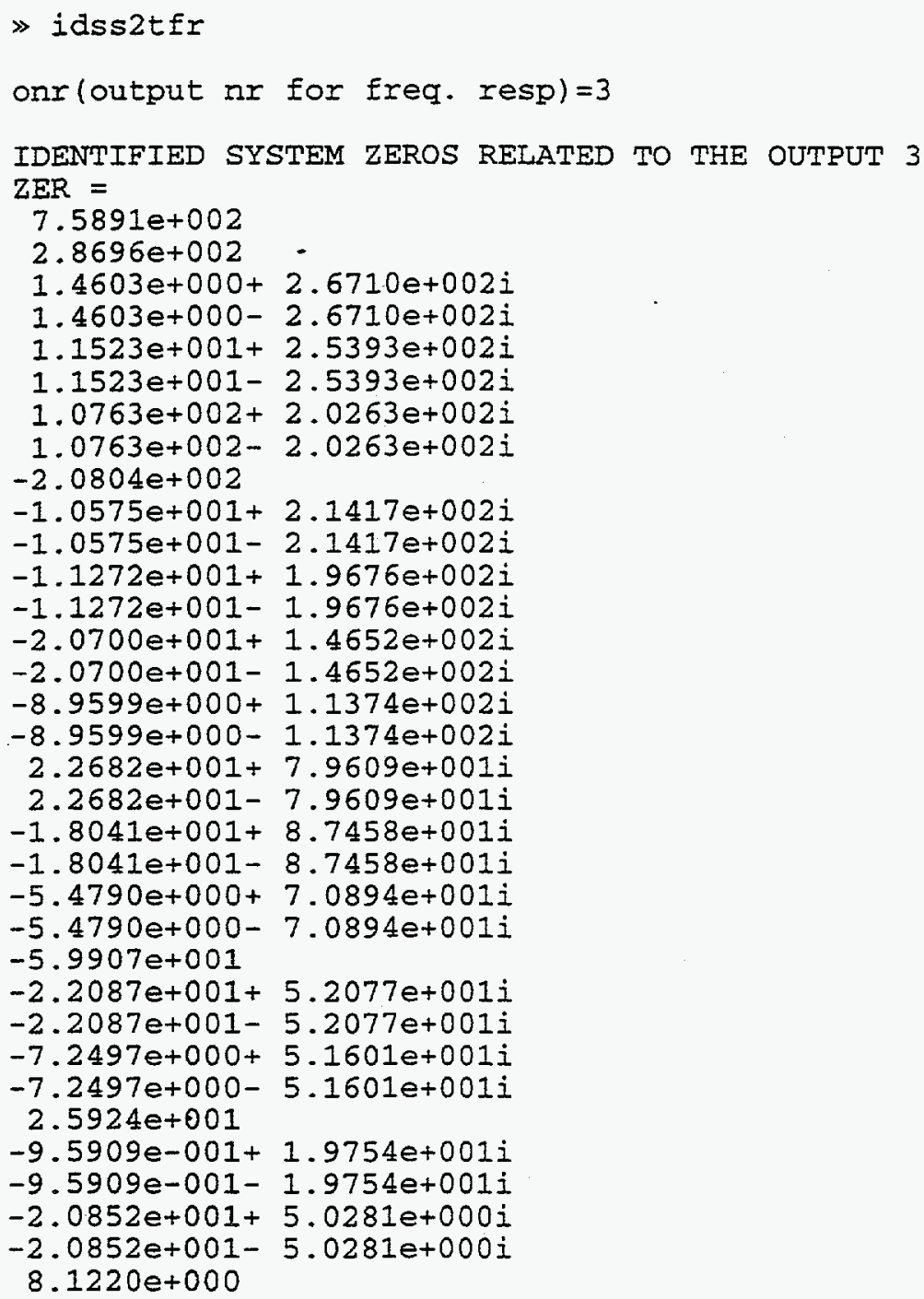




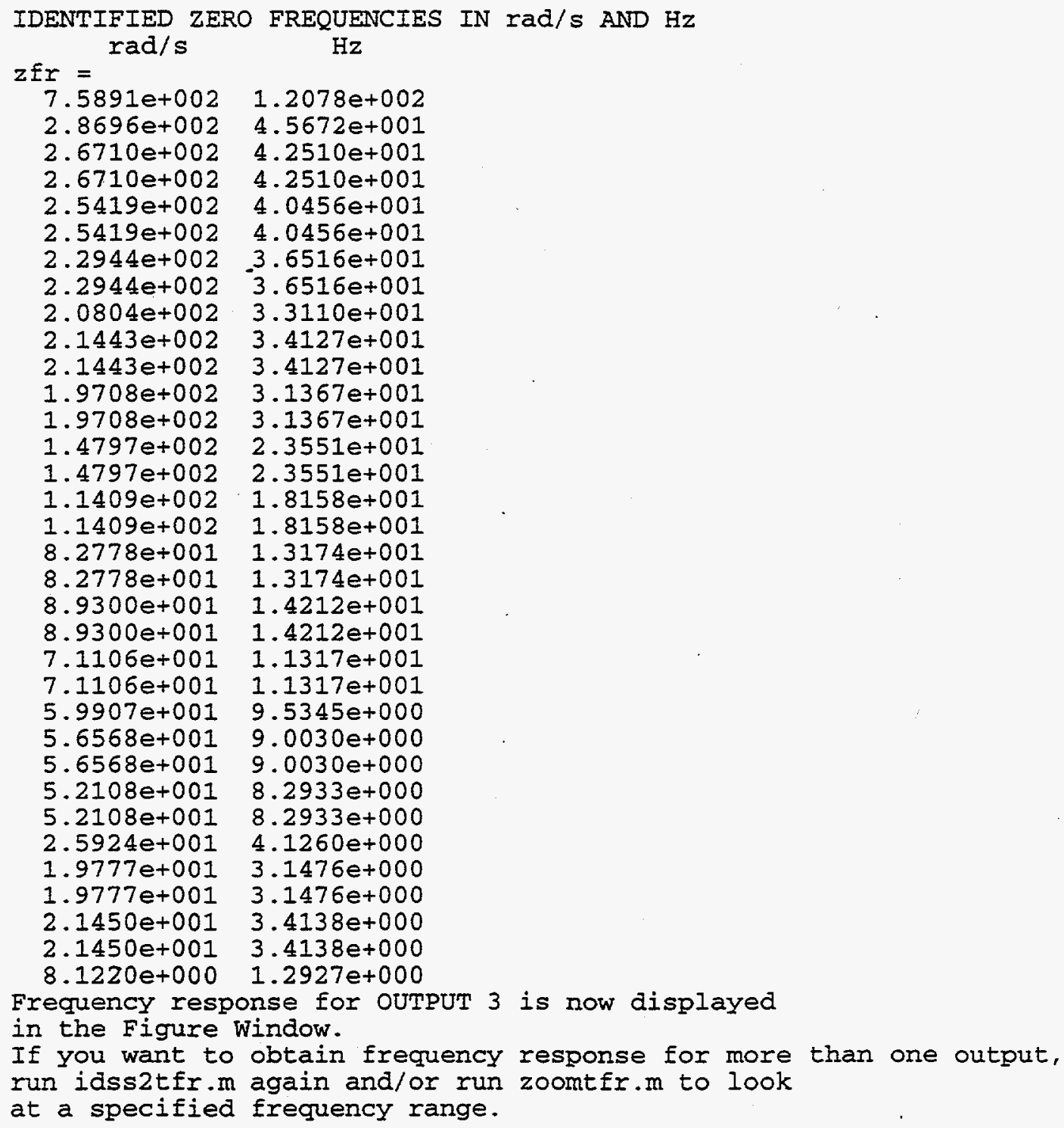


FREQUENCY RESPONSE FOR OUTPUT 3
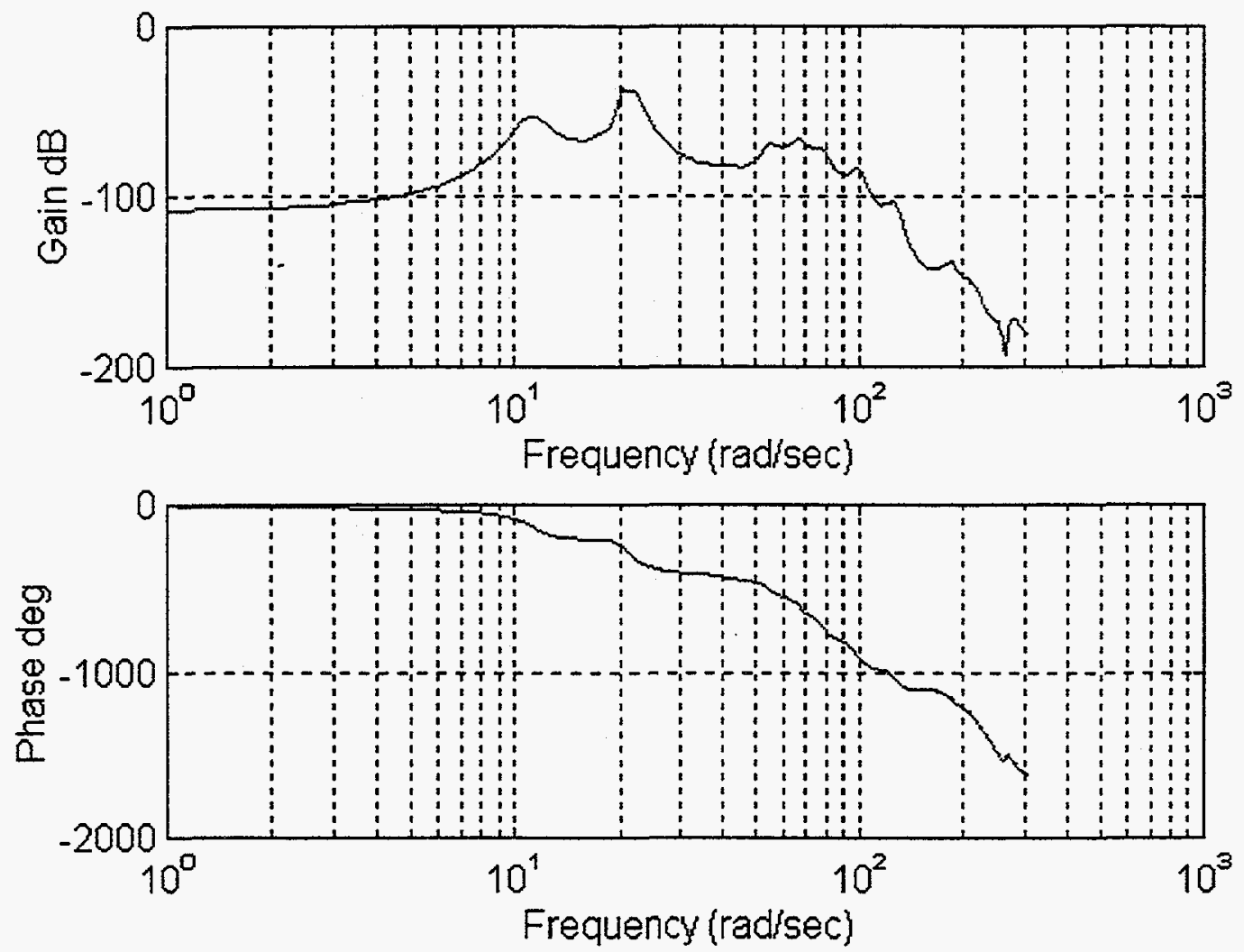

FREQUENCY RESPONSE FOR OUTPUT 3
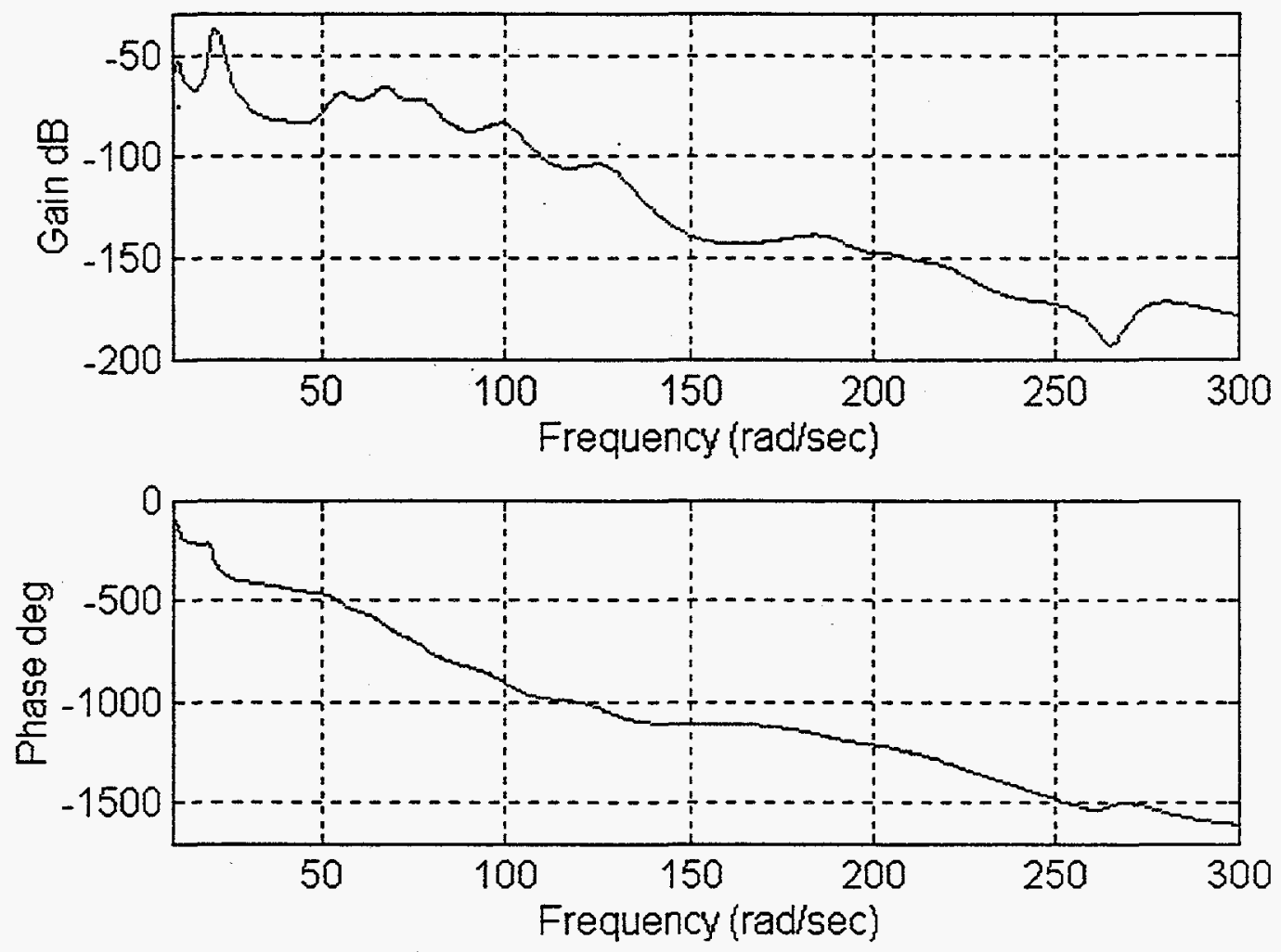


\section{Frequency Response for OUTPUT4}

$\gg$ idss2tfr

onr (output nr for freq. resp) $=4$

IDENTIFIED SYSTEM ZEROS RELATED TO THE OUTPUT 4 ZER =

$3.2648 e+002+7.4147 e+002 i$

$3.2648 e+002-7.4147 e+002 i$

$-4.3054 e+000+2.6499 e+002 i$

$-4.3054 e+000-2.6499 e+002 i$

$-2.2386 e+002$

$6.5682 e+000+2.3892 e+002 i$

$6.5682 \mathrm{e}+000-2.3892 \mathrm{e}+002 i$

$6.1552 e+001+2.1705 e+002 i$

$6.1552 e+001-2.1705 e+002 i$

$-8.6420 e+000+2.0770 e+002 i$

$-8.6420 e+000-2.0770 e+002 i$

$-1.0471 e+001+1.9124 e+002 i$

$-1.0471 e+001-1.9124 e+002 i$

$-2.5244 e+001+1.3929 e+002 i$

$-2.5244 e+001-1.3929 e+002 i$

$-8.6886 e+000+1.1381 e+002 i$

$-8.6886 e+000-1.1381 e+002 i$

$-1.6276 e+001+8.9446 e+001 i$

$-1.6276 e+001-8.9446 e+001 i$

$2.2575 e+001+7.9737 e+001 i$

$2.2575 e+001-7.9737 e+001 i$

$-5.6020 e+000+7.0779 e+001 i$

$-5.6020 e+000-7.0779 e+001 i$

$-5.8216 e+001$

$-1.7071 e+001+5.3976 e+001 i$

$-1.7071 e+001-5.3976 e+001 i$

$-7.9943 e+000+5.1146 e+001 i$

$-7.9943 e+000-5.1146 e+001 i$

$2.8087 e+001$

$-9.3736 e-001+1.8874 e+001 i$

$-9.3736 e-001-1.8874 e+001 i$

$-2.2710 e+001+5.0450 e+000 i$

$-2.2710 e+001-5.0450 e+000 i$

$8.1057 e+000$ 


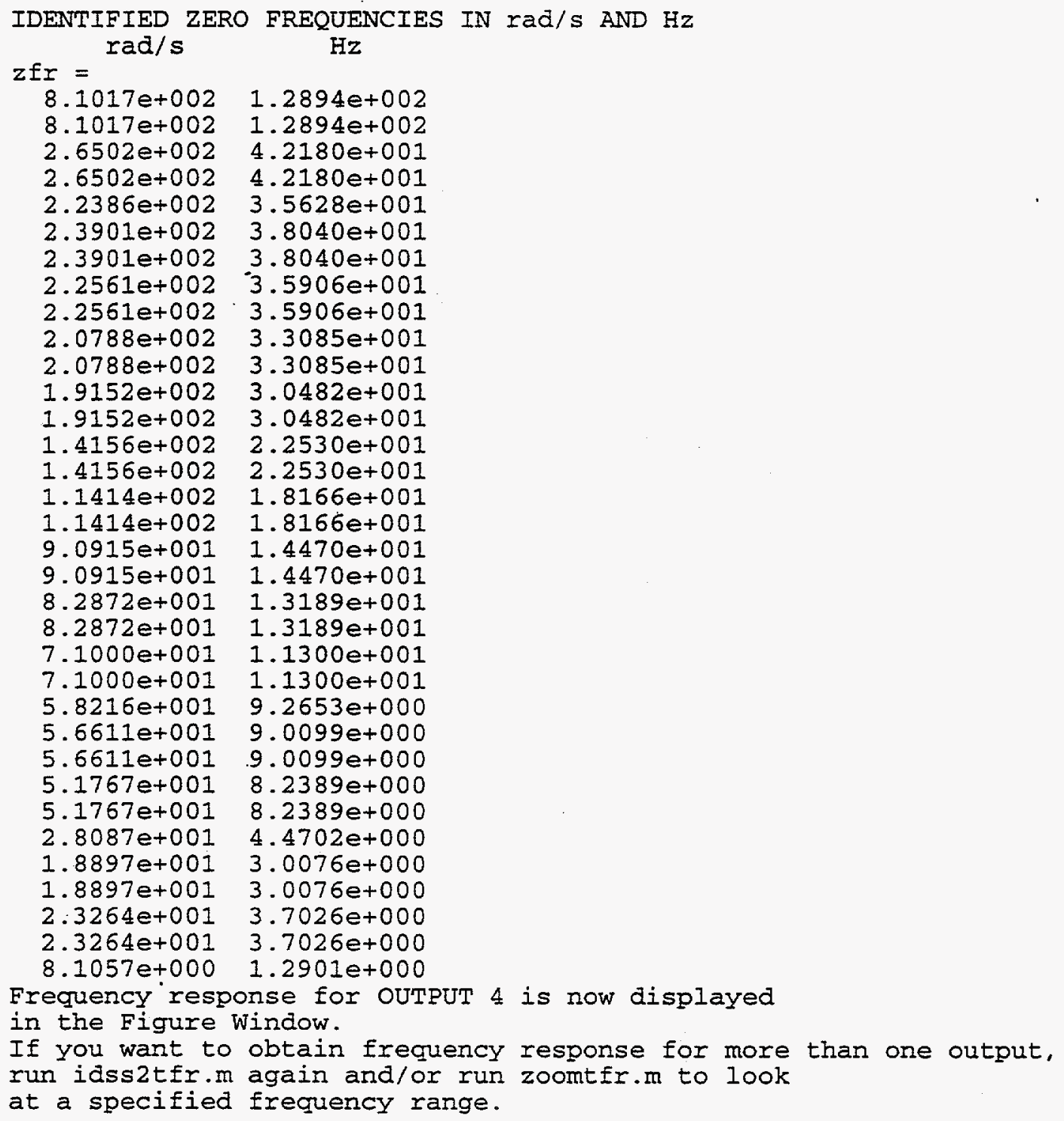



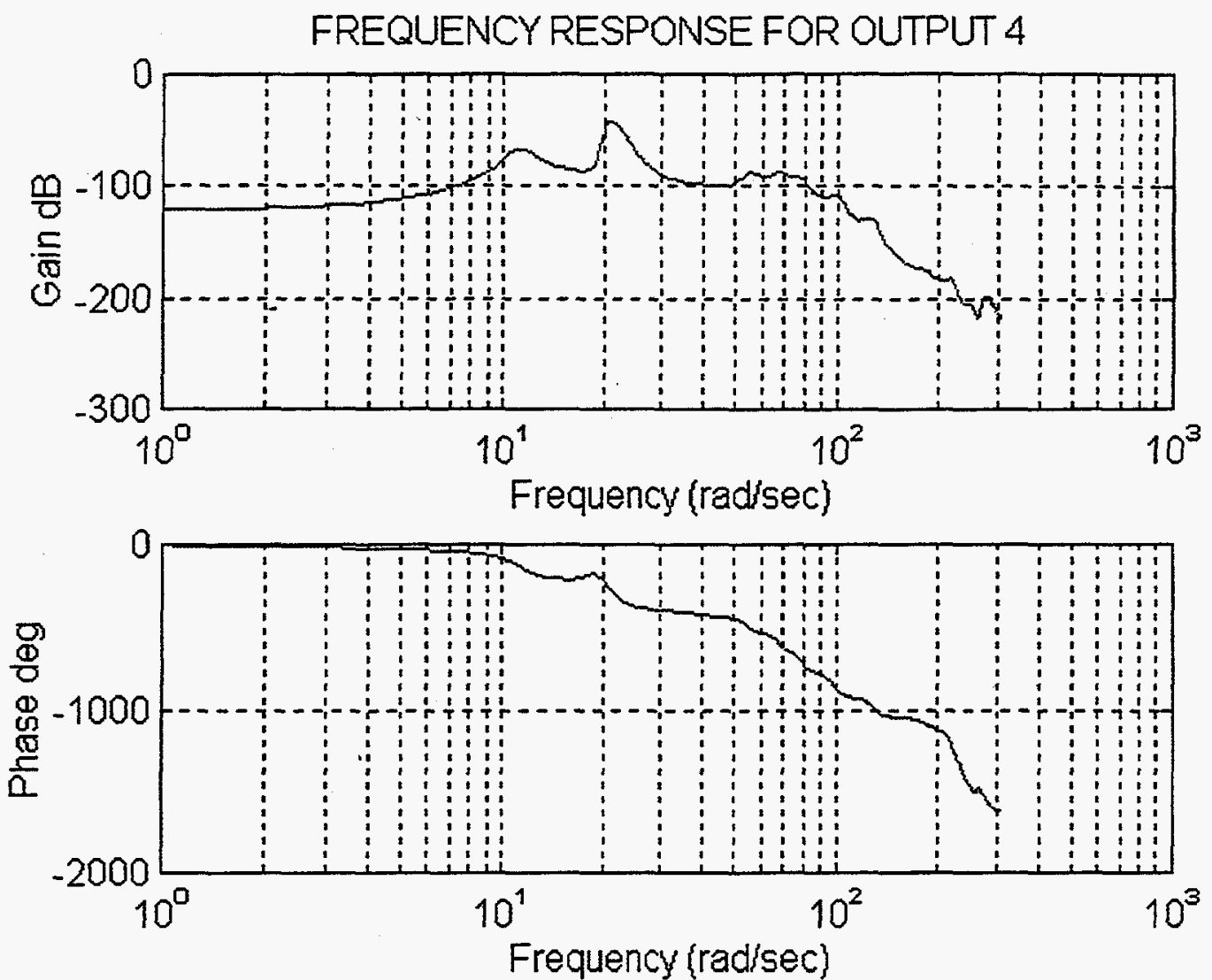

FREQUENCY RESPONSE FOR OUTPUT 4
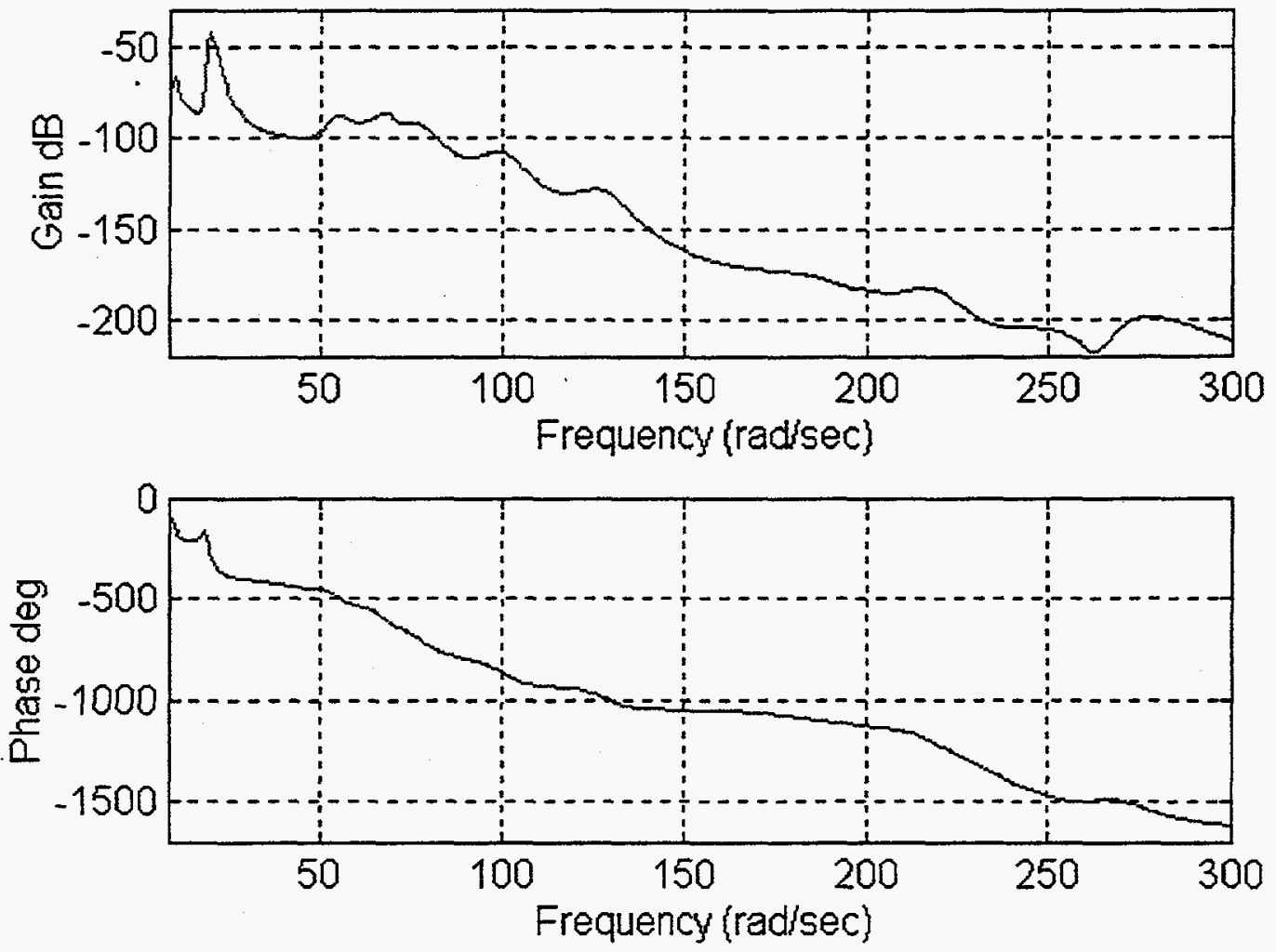


\section{Frequency Response for OUTPUT5}

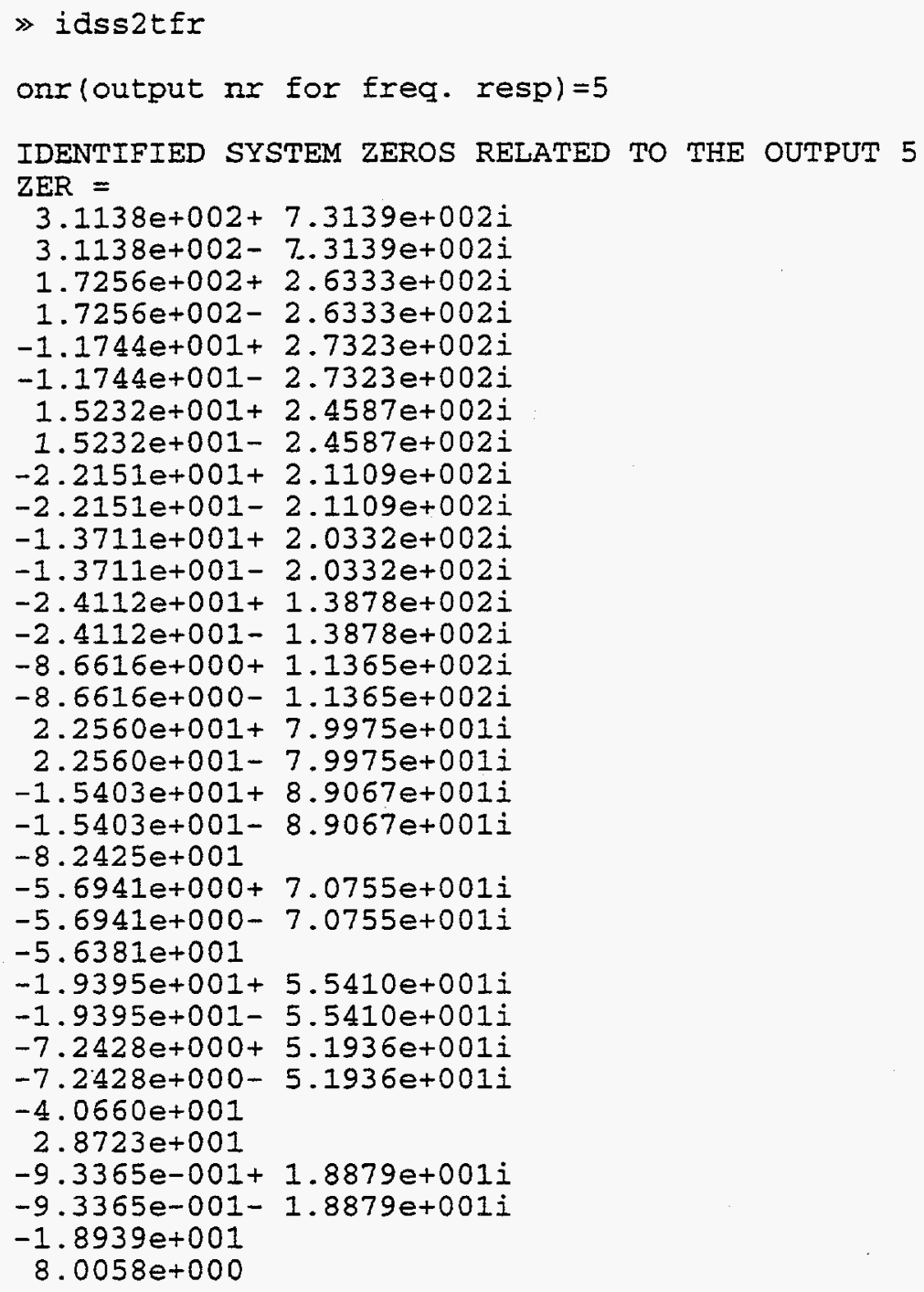




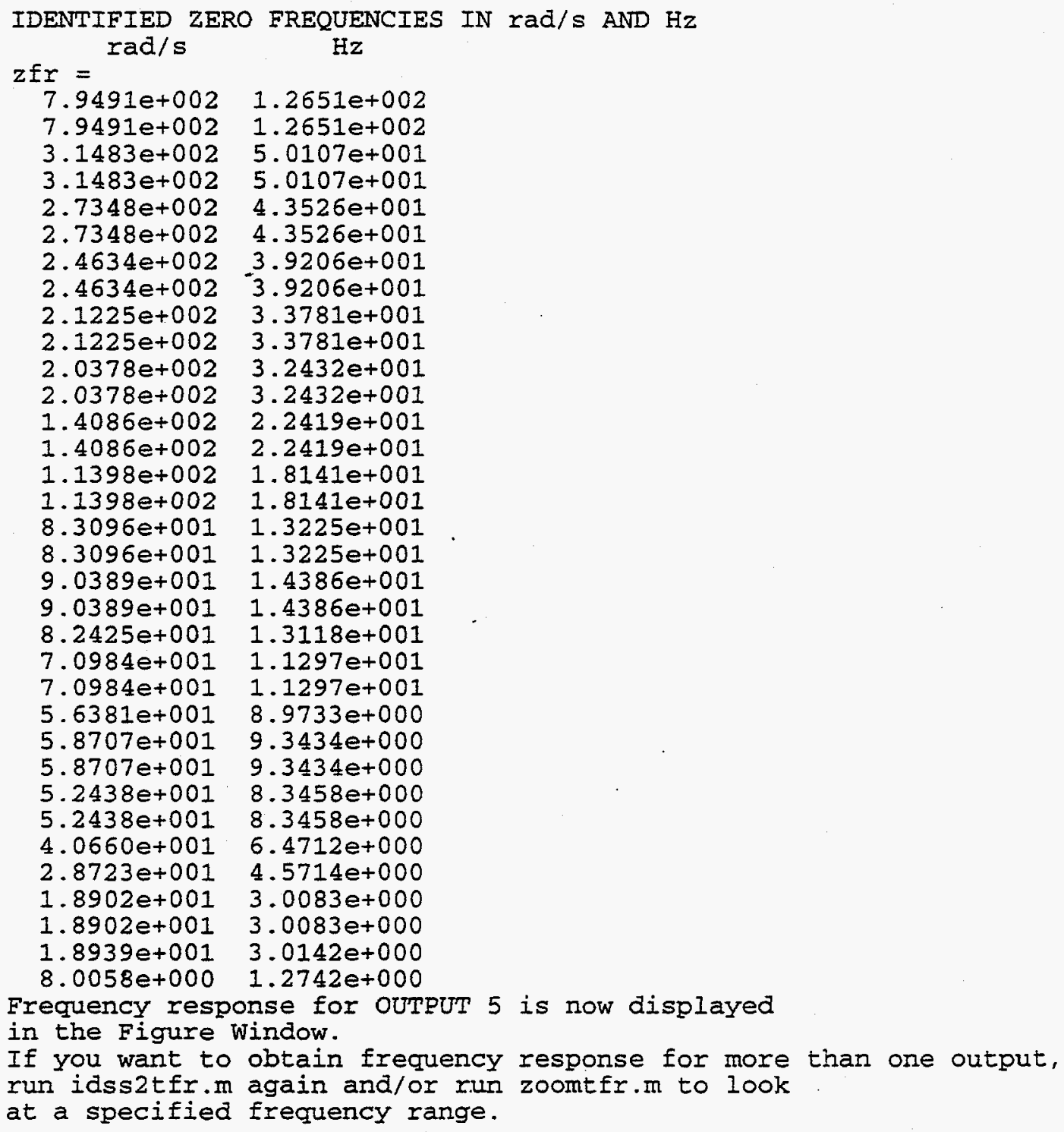


FREQUENCY RESPONSE FOR OUTPUT 5
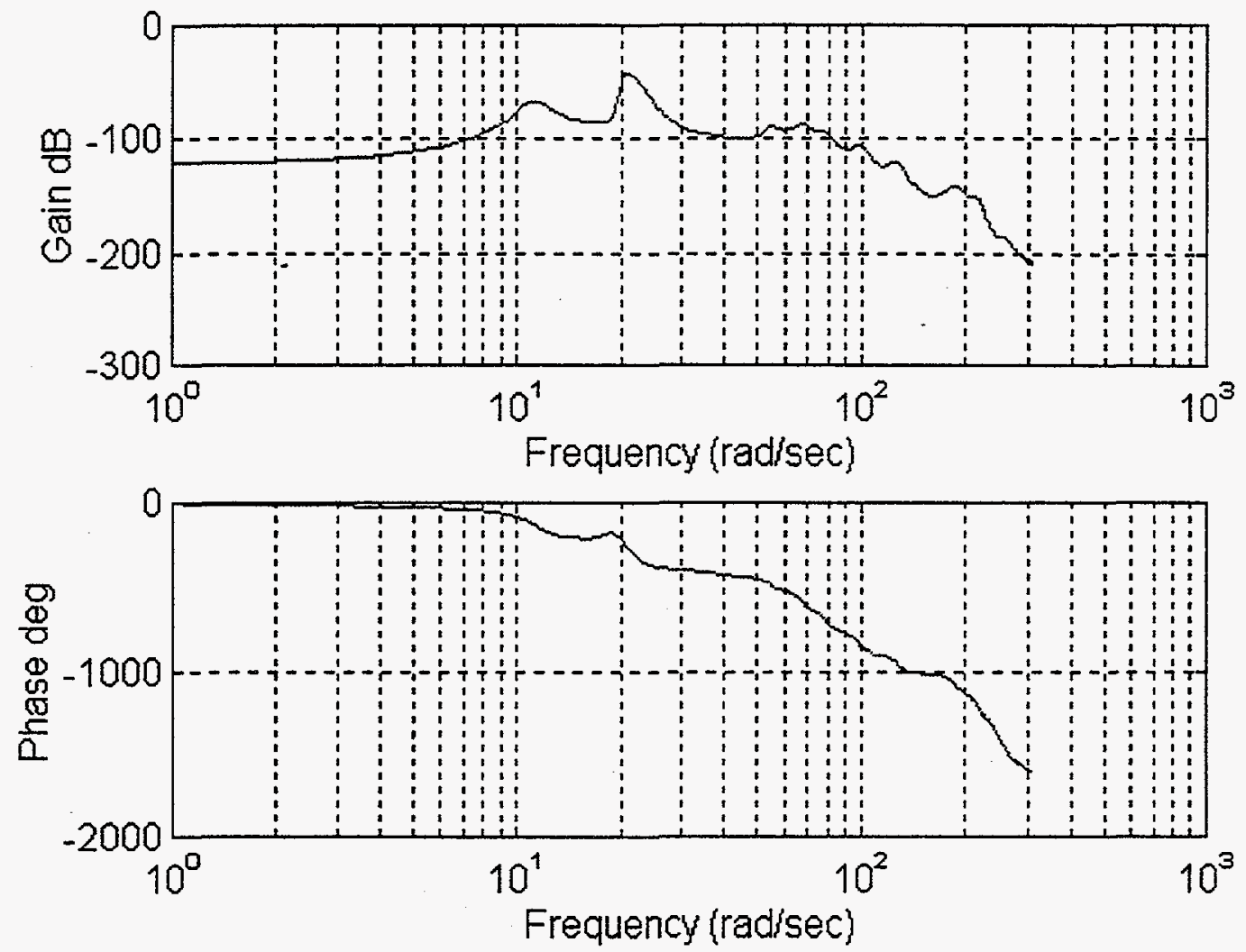

FREQUENCY RESPONSE FOR OUTPUT 5
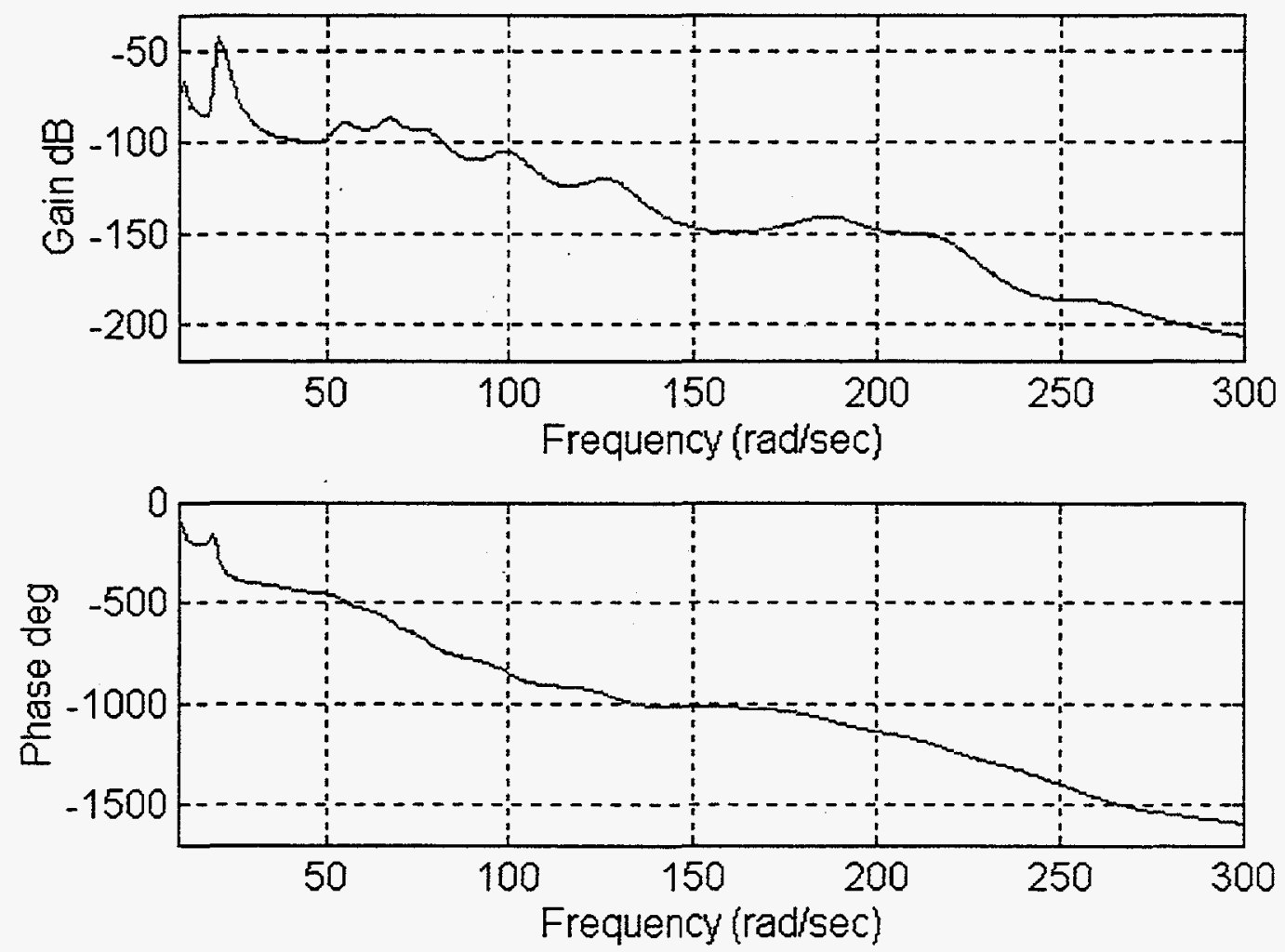


\section{Identification Procedure and Results for the Data File ib20104r.dat}

\section{Loading the Data File}

$\gg$ load ib20104r

$\gg$ whos

$\begin{array}{rrrrrr}\text { Name } & \text { Size } & \text { Elements } & \text { Bytes } & \text { Density } \\ \text { T } & 1 \text { by } 1 & 1 & 8 & \text { FuII } \\ \text { u } & 4092 \text { by } 1 & 4092 & 32736 & \text { FulI } \\ \text { y } & 4092 \text { by } 5 & 20460 & 163680 & \text { Full }\end{array}$

\section{Identification of Observer/Kalman Filter Model}

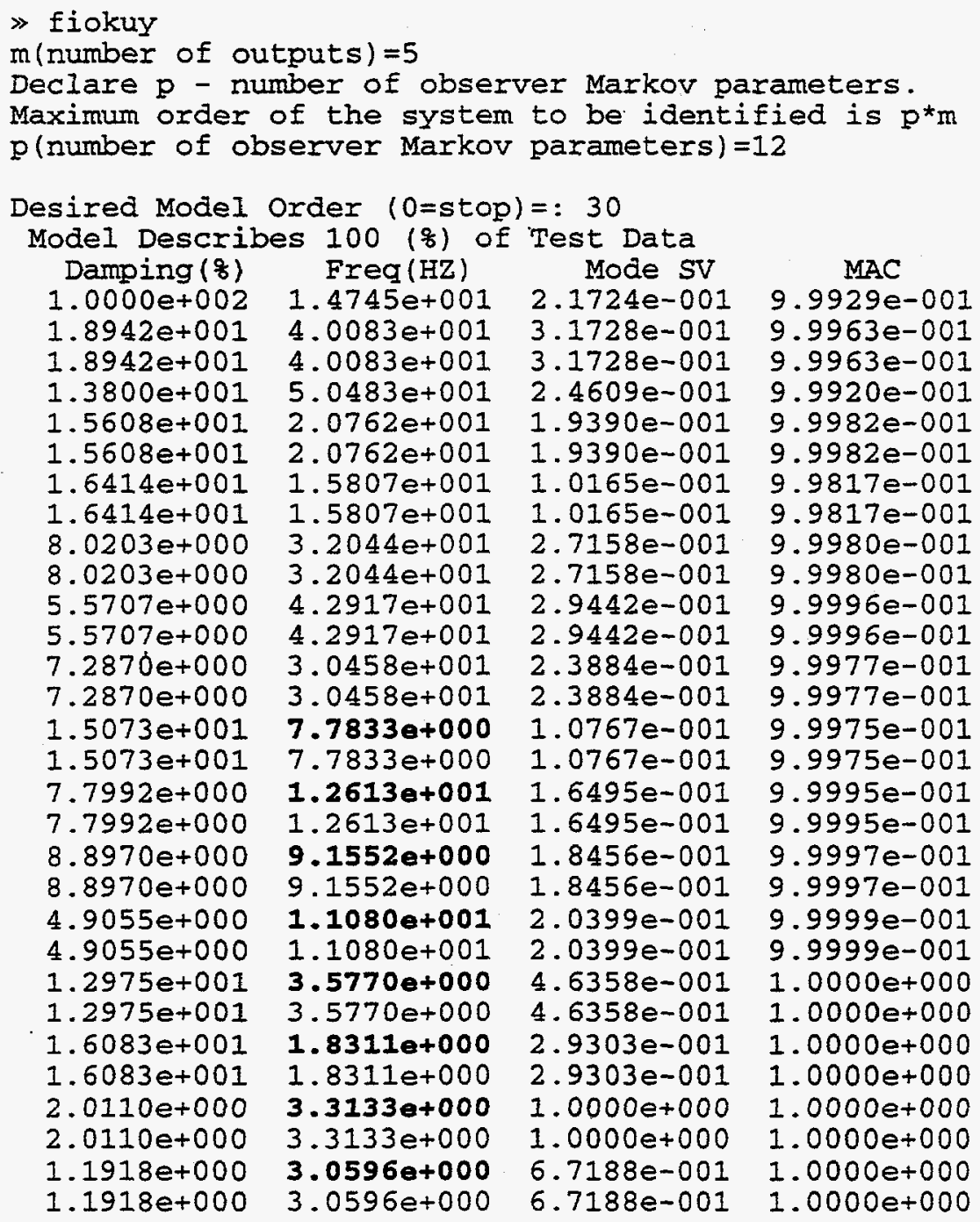

Desired Model Order $(0=s t o p)=: 0$

Identification exror is now displayed in the Figure window Identified system matrices are available as Af, Bf, Cf, Df Observer gain matrix is available as $G$ 

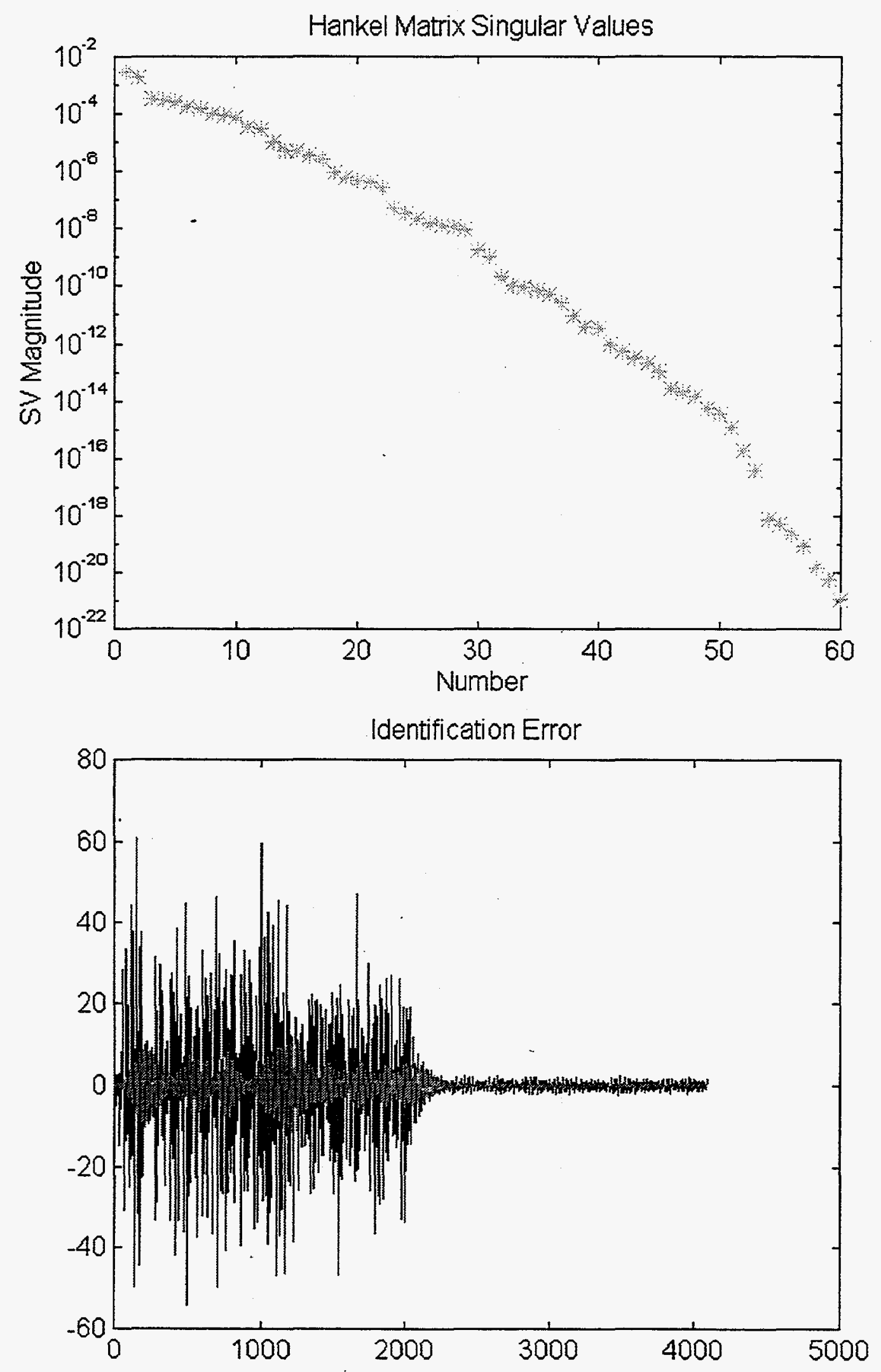


\section{Frequency Response for OUTPUT1}

$\gg$ idss 2 tfr

onr (output $\mathrm{nr}$ for freq. resp) $=1$

IDENTIFIED SYSTEM EIGENVALUES

$r 1=$

$-1.5022 e+001+2.6924 e+002 i$

$-1.5022 e+001-2.6924 e+002 i$

$-4.7704 e+001+2.4729 e+002 i$

$-4.7704 e+001-2.4729 e+002 i$

$-1.6148 e+001+2.0069 e+002 i$

$-1.6148 e+001-2.0069 e+002 i$

$-1.3945 e+001+1.9087 e+002 i$

$-1.3945 e+001-1.9087 e+002 i$

$-2.0361 e+001+1.2885 e+002 i$

$-2.0361 e+001-1.2885 e+002 i$

$-9.2648 e+001$

$-1.6302 e+001+9.7970 e+001 i$

$-1.6302 e+001-9.7970 e+001 i$

$-6.1808 e+000+7.9007 e+001 i$

$-6.1808 e+000-7.9007 e+001 i$

$-3.4152 e+000+6.9536 e+001 i$

$-3.4152 e+000-6.9536 e+001 i$

$-5.1179 e+000+5.7296 e+001 i$

$-5.1179 e+000-5.7296 e+001 i$

$-4.3774 e+001$

$-7.3712 e+000+4.8345 e+001 i$

$-7.3712 e+000-4.8345 e+001 i$

$-2.9162 e+000+2.2285 e+001 i$

$-2.9162 e+000-2.2285 e+001 i$

$-4.1865 e-001+2.0814 e+001 i$

$-4.1865 e-001-2.0814 e+001 i$

$-2.2910 e-001+1.9222 e+001 i$

$-2.2910 e-001-1.9222 e+001 i$

$-1.8504 e+000+1.1355 e+001 i$

$-1.8504 e+000-1.1355 e+001 i$

IDENTIFIED MODAL FREQUENCIES IN rad/s AND $\mathrm{Hz}$ fr $=$

$$
\text { rad/s } \mathrm{Hz}
$$

$2.6965 e+002 \quad 4.2917 e+001$

$2.6965 e+002 \quad 4.2917 e+001$

$2.5185 e+002 \quad 4.0083 e+001$

$2.5185 e+002 \quad 4.0083 e+001$

$2.0134 e+002 \quad 3.2044 e+001$

$2.0134 e+002 \quad 3.2044 e+001$

$1.9137 e+002 \quad 3.0458 e+001$

$1.9137 e+002 \quad 3.0458 e+001$

$1.3045 e+002 \quad 2.0762 e+001$

$1.3045 e+002 \quad 2.0762 e+001$

$9.2648 e+001 \quad 1.4745 e+001$

$9.9317 e+001 \quad 1.5807 e+001$

$9.9317 e+001 \quad 1.5807 e+001$

$7.9248 \mathrm{e}+001 \quad 1.2613 \mathrm{e}+001$

$7.9248 e+001 \quad 1.2613 e+001$

$6.9620 e+001 \quad 1.1080 e+001$

$6.9620 e+001 \quad 1.1080 e+001$

$5.7524 \mathrm{e}+001 \quad 9.1552 \mathrm{e}+000$

$5.7524 e+001 \quad 9.1552 e+000$

$4.3774 e+001 \quad 6.9669 e+000$

$4.8904 e+0017.7833 e+000$

$4.8904 e+001 \quad 7.7833 e+000$

$2.2475 e+001 \quad 3.5770 e+000$ 
$2.2475 e+001 \quad 3.5770 e+000$

$2.0818 e+001 \quad 3.3133 e+000$

$2.0818 e+001 \quad 3.3133 e+000$

$1.9224 \mathrm{e}+001 \quad 3.0596 \mathrm{e}+000$

$1.9224 e+0013.0596 e+000$

$1.1505 e+001 \quad 1.8311 e+000$

$1.1505 e+001 \quad 1.8311 e+000$ 

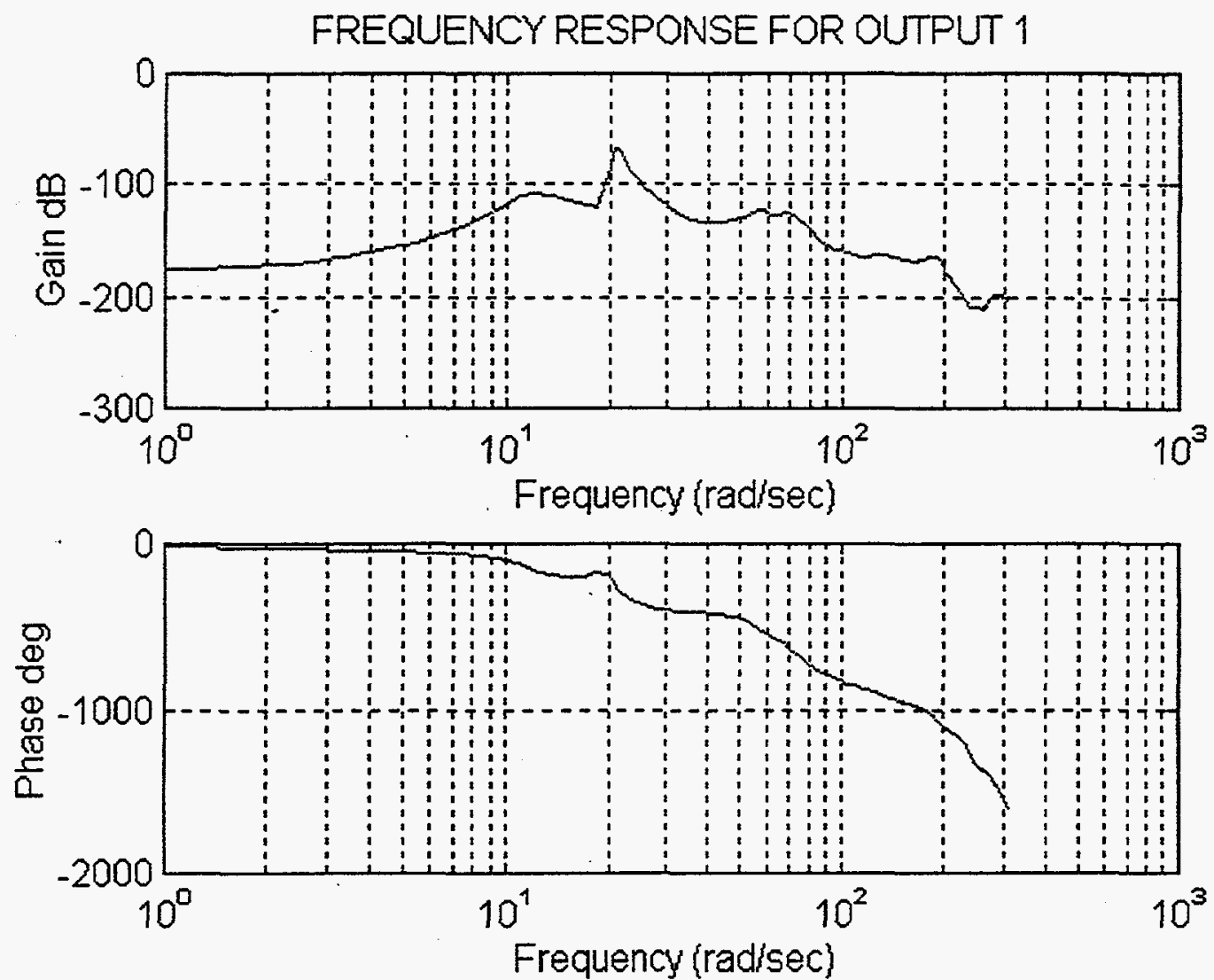

FREQUENCY RESPONSE FOR OUTPUT 1
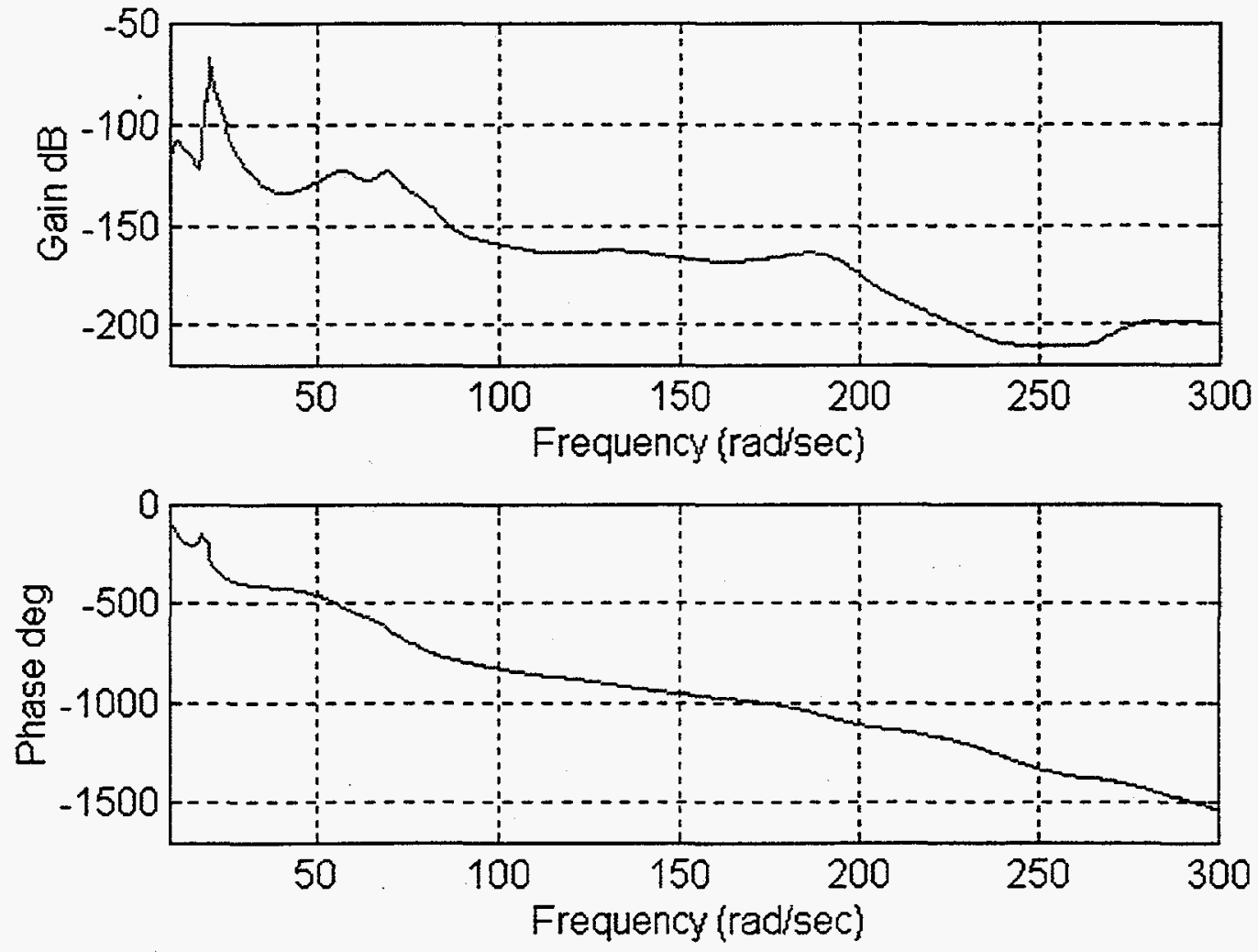


\section{Frequency Response for OUTPUT2}

$\gg$ idss2tfr

onr (output $n r$ for freq. resp) $=2$

IDENTIFIED SYSTEM ZEROS RELATED TO THE OUTPUT 2

$\mathrm{ZER}=$

$-4.3989 e+003$

$-1.7684 e+001+2.6669 e+002 i$

$-1.7684 e+001-2.6669 e+002 i$

$-1.1495 e+001+2.1631 e+002 i$

$-1.1495 e+001-2.1631 e+002 i$

$-1.0905 e+001+1.9932 e+002 i$

$-1.0905 e+001-1.9932 e+002 i$

$-7.1214 \mathrm{e}+001+1.3386 \mathrm{e}+002 i$

$-7.1214 e+001-1.3386 e+002 i$

$-2.4989 e+001+1.1751 e+002 i$

$-2.4989 e+001-1.1751 e+002 i$

$1.2103 e+001+8.4885 e+001 i$

$1.2103 e+001-8.4885 e+001 i$

$-1.3091 e+001+8.6506 e+001 i$

$-1.3091 e+001-8.6506 e+001 i$

$-7.6466 e+000+7.0766 e+001 i$

$-7.6466 e+000-7.0766 e+001 i$

$4.0888 e+001+2.4469 e+001 i$

$4.0888 e+001-2.4469 e+001 i$

$-1.6872 e+001+5.2591 e+001 i$

$-1.6872 e+001-5.2591 e+001 i$

$-1.0618 e+001+5.0714 e+001 i$

$-1.0618 e+001-5.0714 e+001 i$

$-3.9884 e+001$

$-8.8308 e-001+1.9410 e+001 i$

$-8.8308 e-001-1.9410 e+001 i$

$-6.9468 e-001+1.8881 e+001 i$

$-6.9468 e-001-1.8881 e+001 i$

$-6.4927 e+000$

$2.5081 e+000$ 
IDENTIFIED ZERO FREQUENCIES IN rad/s AND $\mathrm{Hz}$ $z$ fr $=$

$4.3989 e+0037.0010 e+002$

$2.6728 e+002 \quad 4.2539 e+001$

$2.6728 e+002 \quad 4.2539 e+001$

$2.1661 e+002 \quad 3.4475 e+001$

$2.1661 e+002 \quad 3.4475 e+001$

$1.9962 \mathrm{e}+002 \quad 3.1770 \mathrm{e}+001$

$1.9962 \mathrm{e}+002 \quad 3.1770 \mathrm{e}+001$

$1.5162 e+002 \quad 2.4131 e+001$

$1.5162 e+002 \quad 2.4131 e+001$

$1.2013 e+002$ : $1.9120 e+001$

$1.2013 e+002 \quad 1.9120 e+001$

$8.5743 e+001 \quad 1.3646 e+001$

$8.5743 e+001 \quad 1.3646 e+001$

$8.7491 e+001 \quad 1.3925 e+001$

$8.7491 e+001 \quad 1.3925 e+001$

$7.1178 \mathrm{e}+001 \quad 1.1328 \mathrm{e}+001$

$7.1178 e+001 \quad 1.1328 e+001$

$4.7651 e+0017.5839 e+000$

$4.7651 e+001 \quad 7.5839 e+000$

$5.5232 e+001 \quad 8.7904 e+000$

$5.5232 e+001 \quad 8.7904 e+000$

$5.1814 e+001 \quad 8.2464 e+000$

$5.1814 e+001 \quad 8.2464 e+000$

$3.9884 e+001 \quad 6.3477 e+000$

$1.9430 \mathrm{e}+001 \quad 3.0924 \mathrm{e}+000$

$1.9430 \mathrm{e}+001 \quad 3.0924 \mathrm{e}+000$

$1.8893 e+001 \quad 3.0070 e+000$

$1.8893 e+001 \quad 3.0070 e+000$

$6.4927 e+000 \quad 1.0333 e+000$

$2.5081 e+000 \quad 3.9917 e-001$

Frequency response for OUTPUT 2 is now displayed in the Figure Window.

If you want to obtain frequency response for more than one output, run idss2tfr.m again and/or run zoomtfr.m to look at a specified frequency range. 

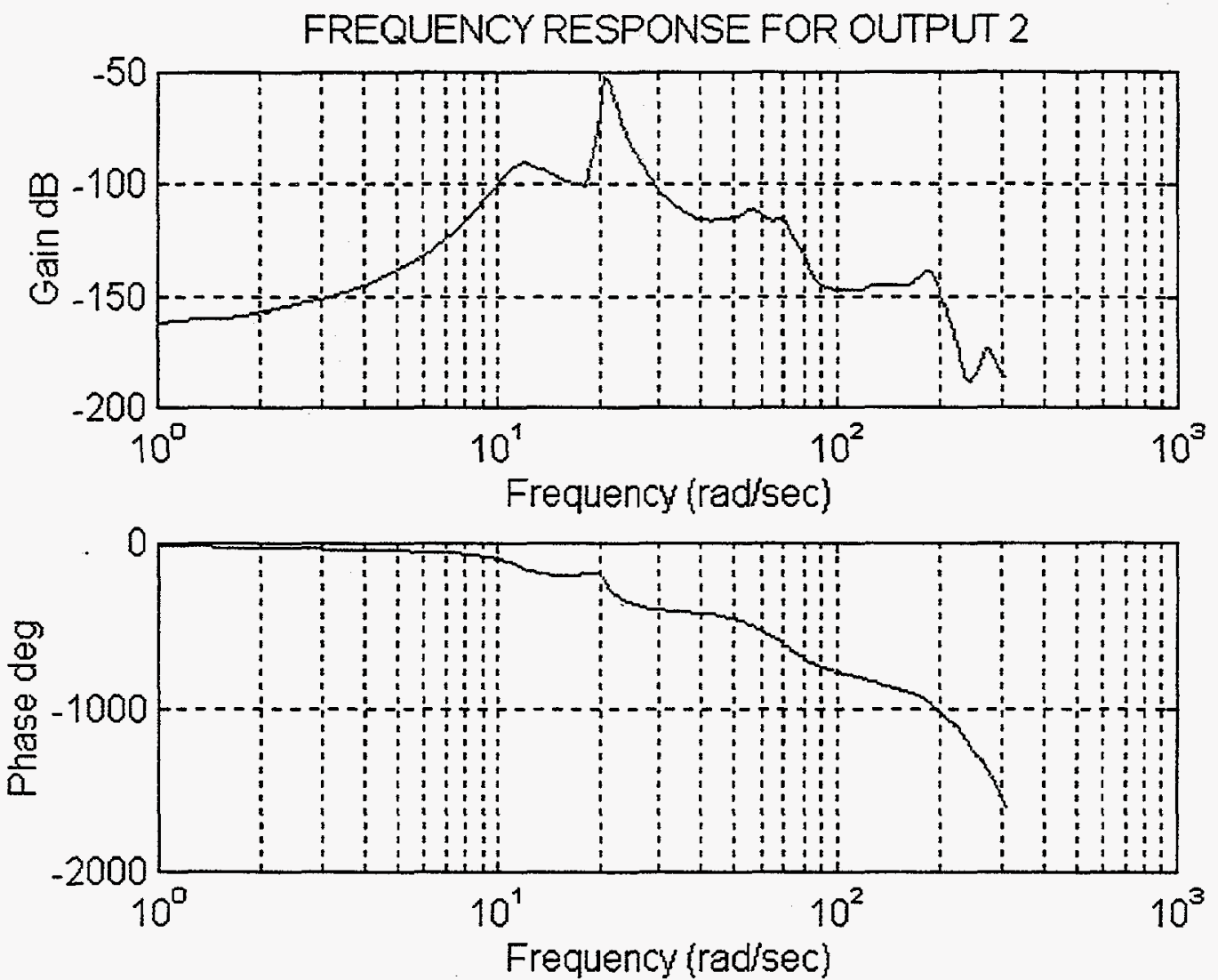

FREQUENCY RESPONSE FOR OUTPUT 2
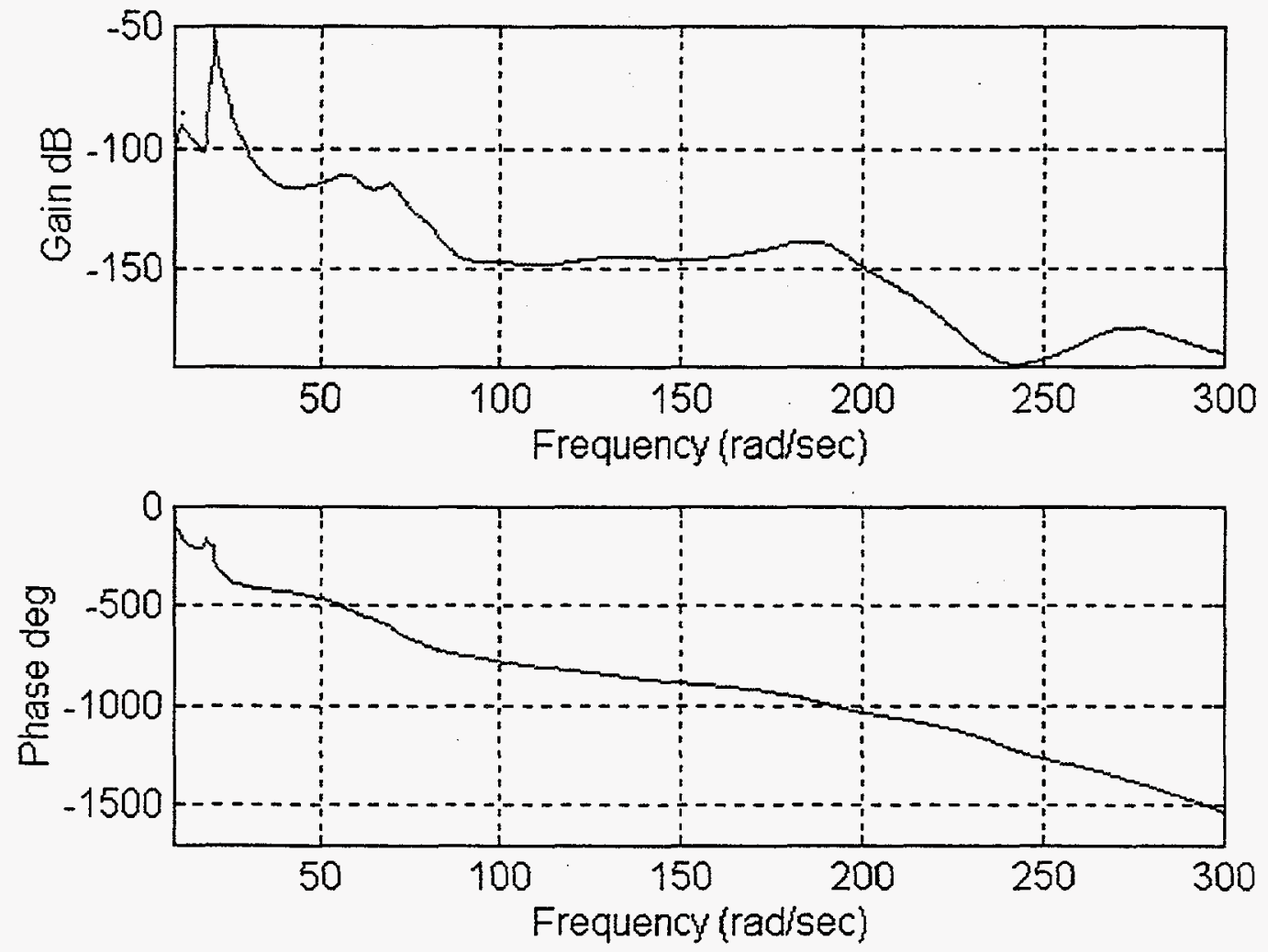


\section{Frequency Response for OUTPUT3}

$\gg i d s s 2 t f r$

onr (output $\mathrm{nr}$ for freq. resp) $=3$

IDENTIFIED SYSTEM ZEROS RELATED TO THE OUTPUT 3 $\mathrm{ZER}=$

$8.6326 e+002$

$-6.4073 e+000+2.6398 e+002 i$

$-6.4073 e+000-2.6398 e+002 i$

$-1.9152 e+002$

$-1.1353 e+001+2.0606 e+002 i$

$-1.1353 e+001-2.0606 e+002 i$

$2.6660 e+001+1.7390 e+002 i$

$2.6660 e+001-1.7390 e+002 i$

$-1.8334 \mathrm{e}+001+1.6454 \mathrm{e}+002 i$

$-1.8334 e+001-1.6454 e+002 i$

$1.0484 \mathrm{e}+002$

$6.7411 e+001+9.0721 e+001 i$

$6.7411 e+001-9.0721 e+001 i$

$-2.0537 e+001+1.1221 e+002 i$

$-2.0537 e+001-1.1221 e+002 i$

$-1.1376 e+001+7.9930 e+001 i$

$-1.1376 e+001-7.9930 \mathrm{e}+001 i$

$2.6052 e+001+6.6865 e+001 i$

$2.6052 e+001-6.6865 e+001 i$

$-4.3307 e+001$

$-1.0481 e+001+5.2750 e+001 i$

$-1.0481 e+001-5.2750 e+001 i$

$-1.5555 e+001+4.6278 e+001 i$

$-1.5555 e+001-4.6278 e+001 i$

$-8.4695 e-001+1.9186 e+001 i$

$-8.4695 e-001-1.9186 e+001 i$

$-1.1740 e+001$

$7.9073 e+000$

$-7.7936 e-001+5.1476 e-001 i$

$-7.7936 e-001-5.1476 e-001 i$ 


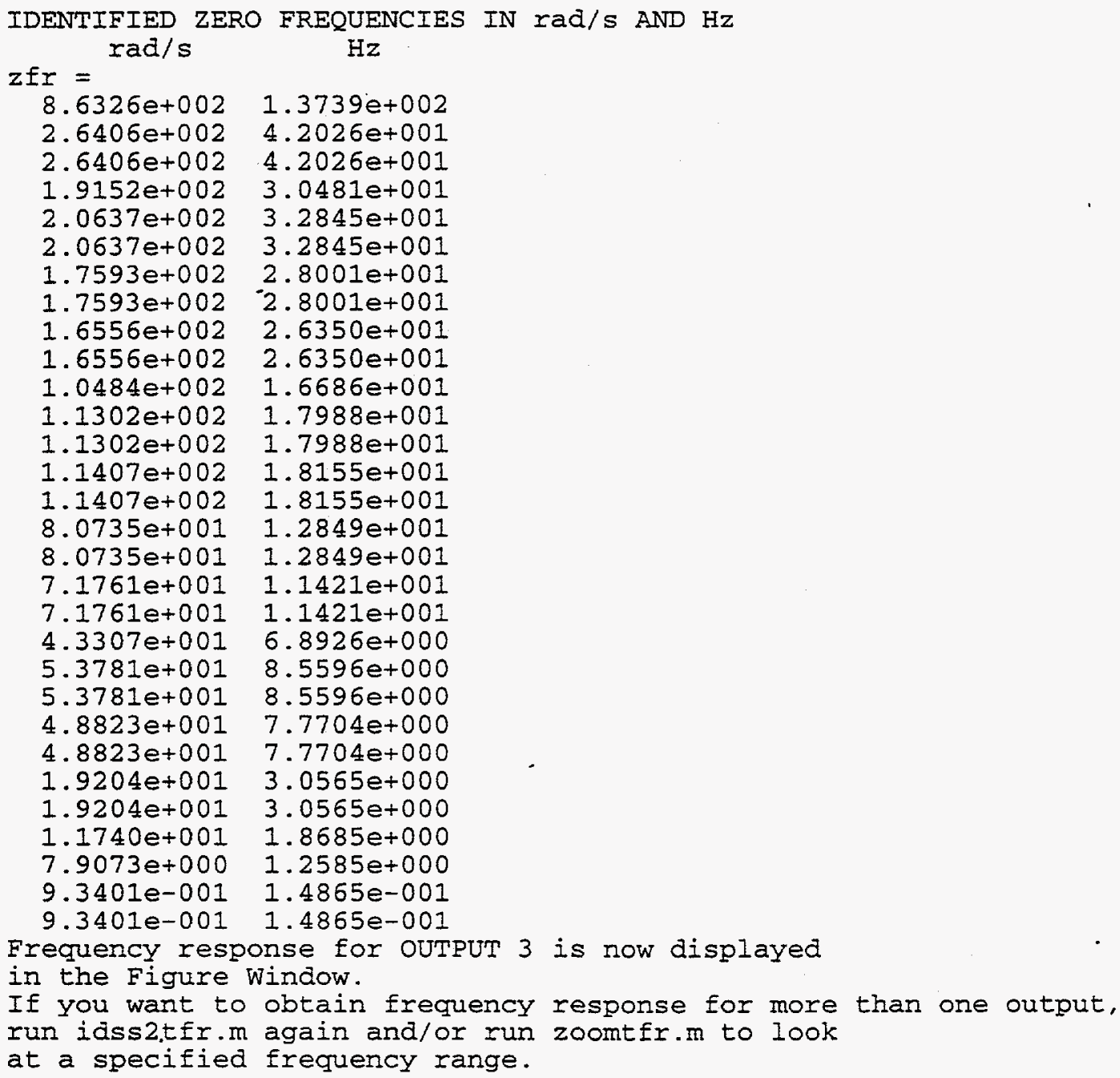



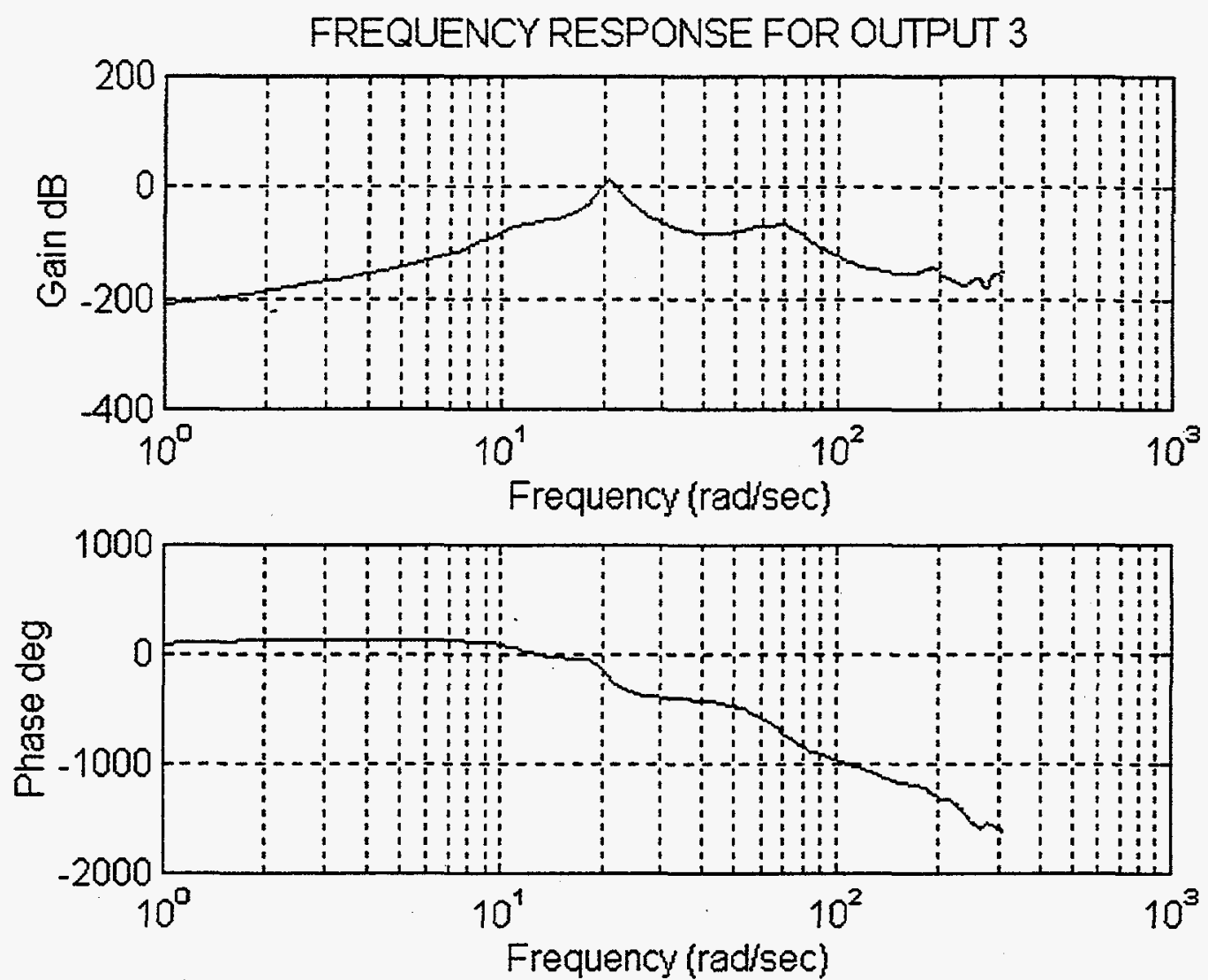

FREQUENCY RESPONSE FOR OUTPUT 3
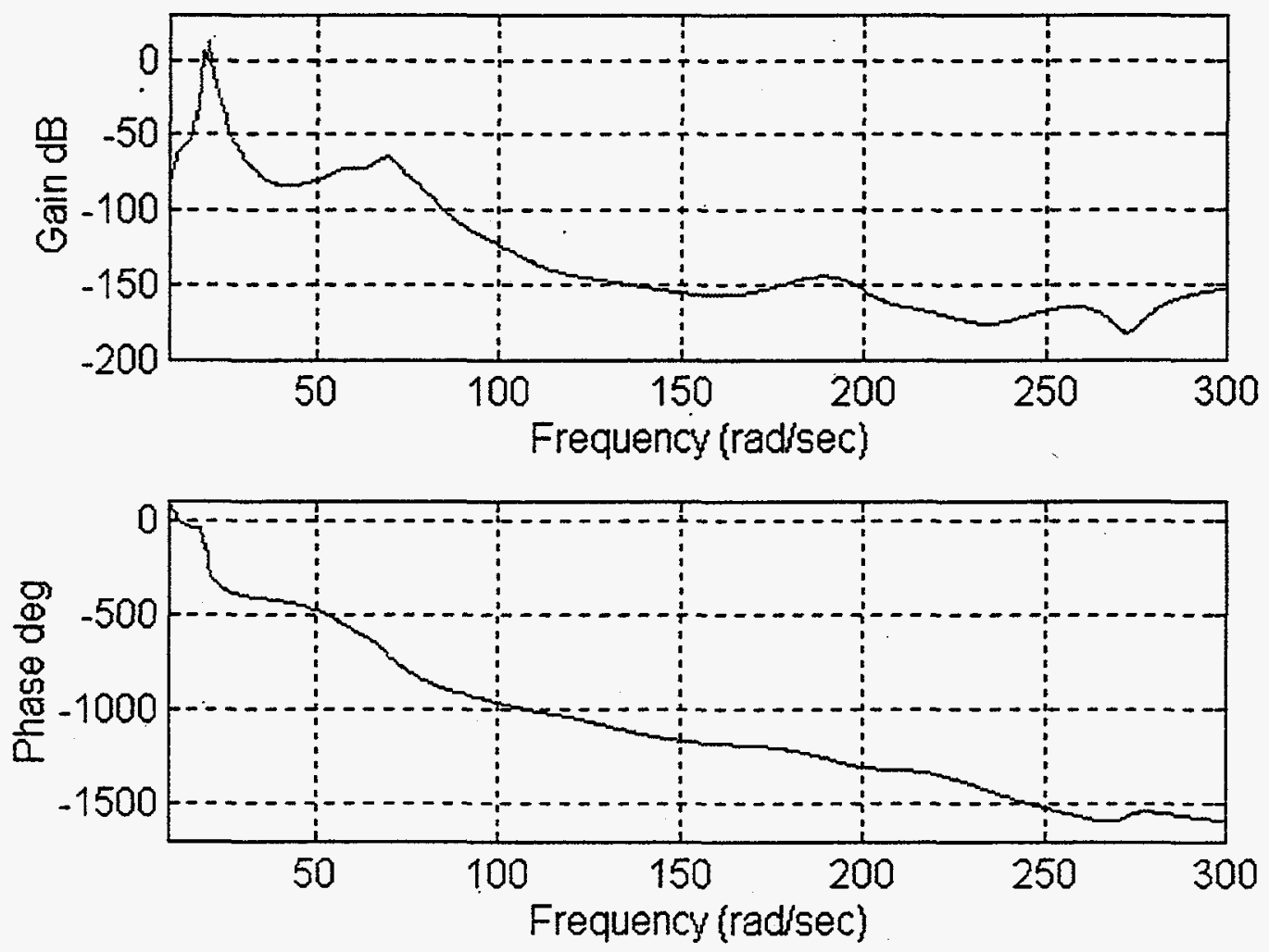


\section{Frequency Response for OUTPUT4 (Collocation Case)}

$\gg i d s s 2 t f r$

onr (output $n x$ for freq. resp) $=4$

IDENTIFIED SYSTEM ZEROS RELATED TO THE OUTPUT 4 $\mathrm{ZER}=$

$-2.3327 e+003$

$-3.5061 e+000+2.8603 e+002 i$

$-3.5061 e+000-2.8603 e+002 i$

$-6.1002 e+000+2.2528 e+002 i$

$-6.1002 e+000-2.2528 e+002 i$

$-1.4626 e+001+1.9473 e+002 i$

$-1.4626 e+001-1.9473 e+002 i$

$-3.0159 e+001+1.4704 e+002 i$

$-3.0159 e+001-1.4704 e+002 i$

$-1.0888 e+001+1.0021 e+002 i$

$-1.0888 e+001-1.0021 e+002 i$

$6.4382 e+000+8.0667 e+001 i$

$6.4382 e+000-8.0667 e+001 i$

$-2.2426 e+001+7.7991 e+001 i$

$-2.2426 e+001-7.7991 e+001 i$

$-6.3846 e+000+7.0589 e+001 i$

$-6.3846 e+000-7.0589 e+001 i$

$-5.1739 e+000+5.4022 e+001 i$

$-5.1739 e+000-5.4022 e+001 i$

$-8.7442 e+000+4.4793 e+001 i$

$-8.7442 e+000-4.4793 e+001 i$

$-2.5653 e+001+1.3899 e+001 i$

$-2.5653 e+001-1.3899 e+001 i$

$-8.9731 e-001+2.0636 e+001 i$

$-8.9731 e-001-2.0636 e+001 i$

$-7.6275 e-001+1.9247 e+001 i$

$-7.6275 e-001-1.9247 e+001 i$

$-8.8440 e-001+1.1182 e+001 i$

$-8.8440 e-001-1.1182 e+001 i$

$2.7452 e+000$ 


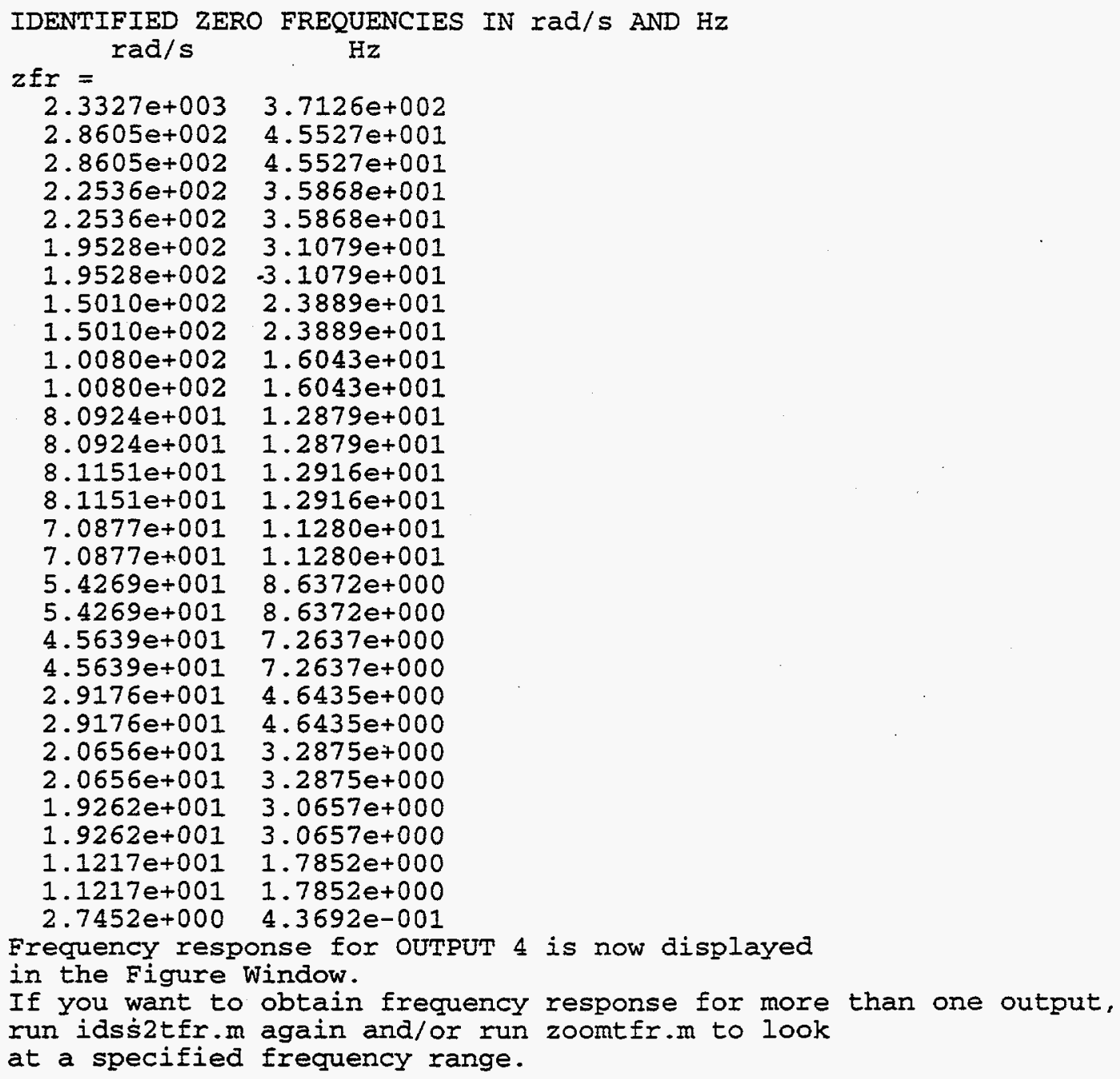



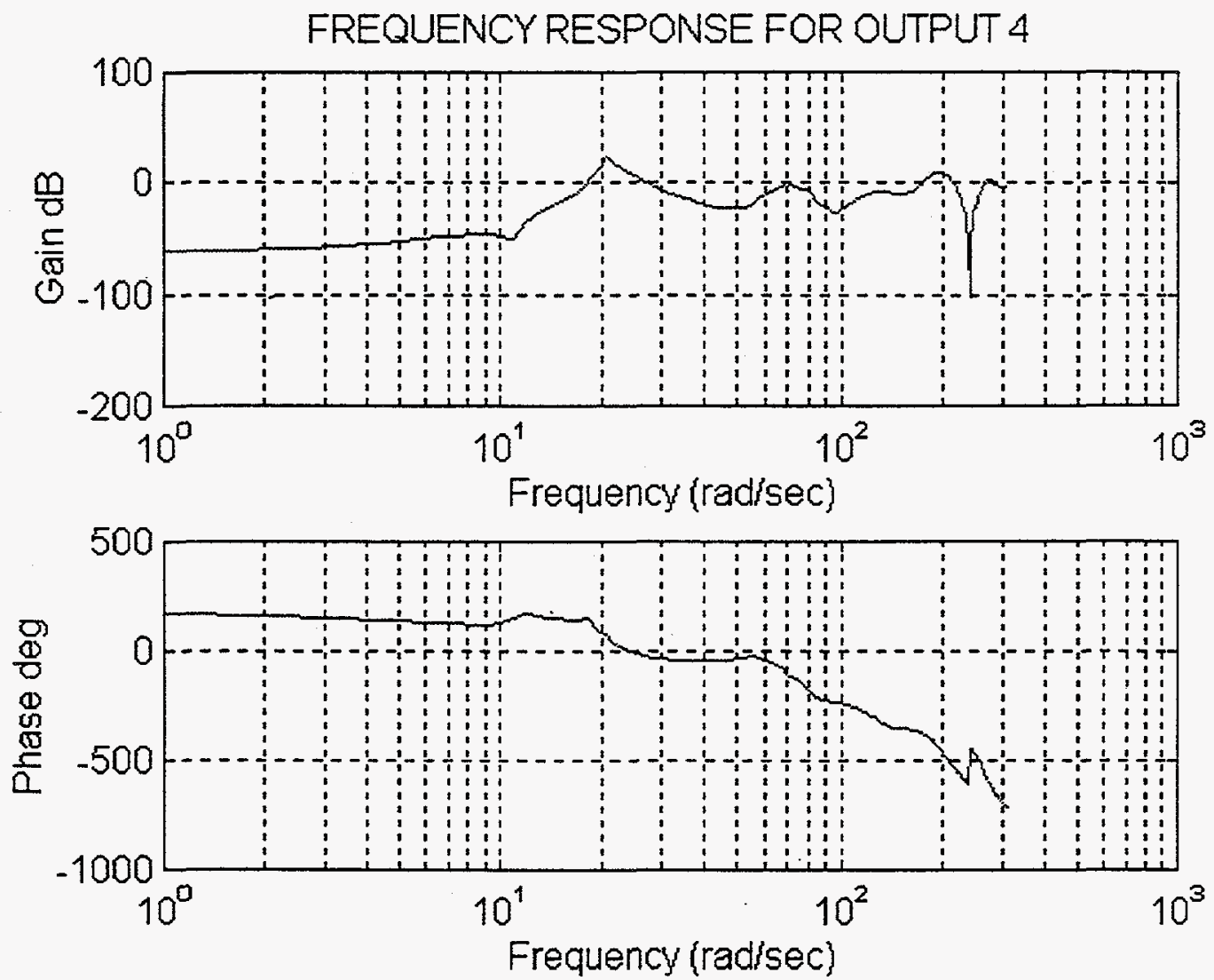

FREQUENCY RESPONSE FOR OUTPUT 4
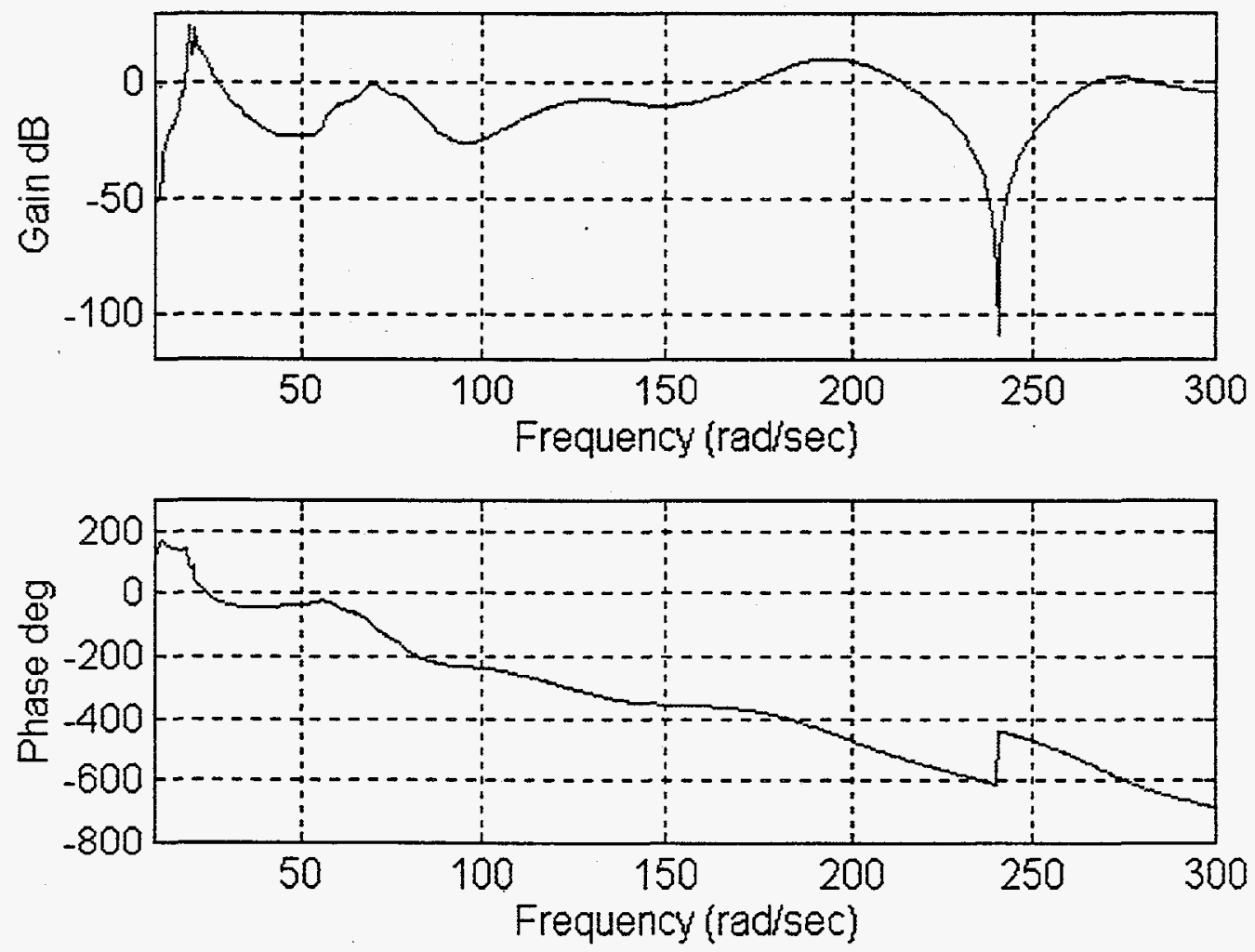
Frequency Response for OUTPUT5

$\gg$ idss2tfr

onr (output $\mathrm{nr}$ for freq. resp) $=5$

IDENTIFIED SYSTEM ZEROS RELATED TO THE OUTPUT 5 $\mathrm{ZER}=$

$4.4886 \mathrm{e}+003$

$-1.1472 e+001+2.6860 e+002 i$

$-1.1472 e+001-2.6860 e+002 i$

$-1.9495 \mathrm{e}+002$

$-1.1341 \mathrm{e}+001+2.1284 \mathrm{e}+002 i$

$-1.1341 e+001-2.1284 e+002 i$

$-3.0163 e-001+1.9309 e+002 i$

$-3.0163 e-001-1.9309 e+002 i$

$-1.6254 \mathrm{e}+001+1.5784 \mathrm{e}+002 i$

$-1.6254 e+001-1.5784 e+002 i$

$-2.0109 e+001+1.1315 e+002 i$

$-2.0109 e+001-1.1315 e+002 i$

$2.6227 e+001+9.5138 e+0.01 i$

$2.6227 e+001-9.5138 e+001 i$

$-1.1433 e+001+7.9941 e+001 i$

$-1.1433 e+001-7.9941 e+001 i$

$2.4203 e+001+6.1282 e+001 i$

$2.4203 e+001-6.1282 e+001 i$

$5.1902 e+001$

$-4.4726 e \div 001$

$-1.0198 e+001+5.2411 e+001 i$

$-1.0198 e+001-5.2411 e+001 i$

$-1.5897 e+001+4.5588 e+001 i$

$-1.5897 e+001-4.5588 e+001 i$

$-8.6748 \mathrm{e}-001+1.9478 \mathrm{e}+001 i$

$-8.6748 e-001-1.9478 e+001 i$

$1.6752 \mathrm{e}+000+5.6820 \mathrm{e}+000 i$

$1.6752 \dot{e}+000-5.6820 e+000 i$

$-3.8205 e+000+3.4133 e+000 i$

$-3.8205 e+000-3.4133 e+000 i$ 


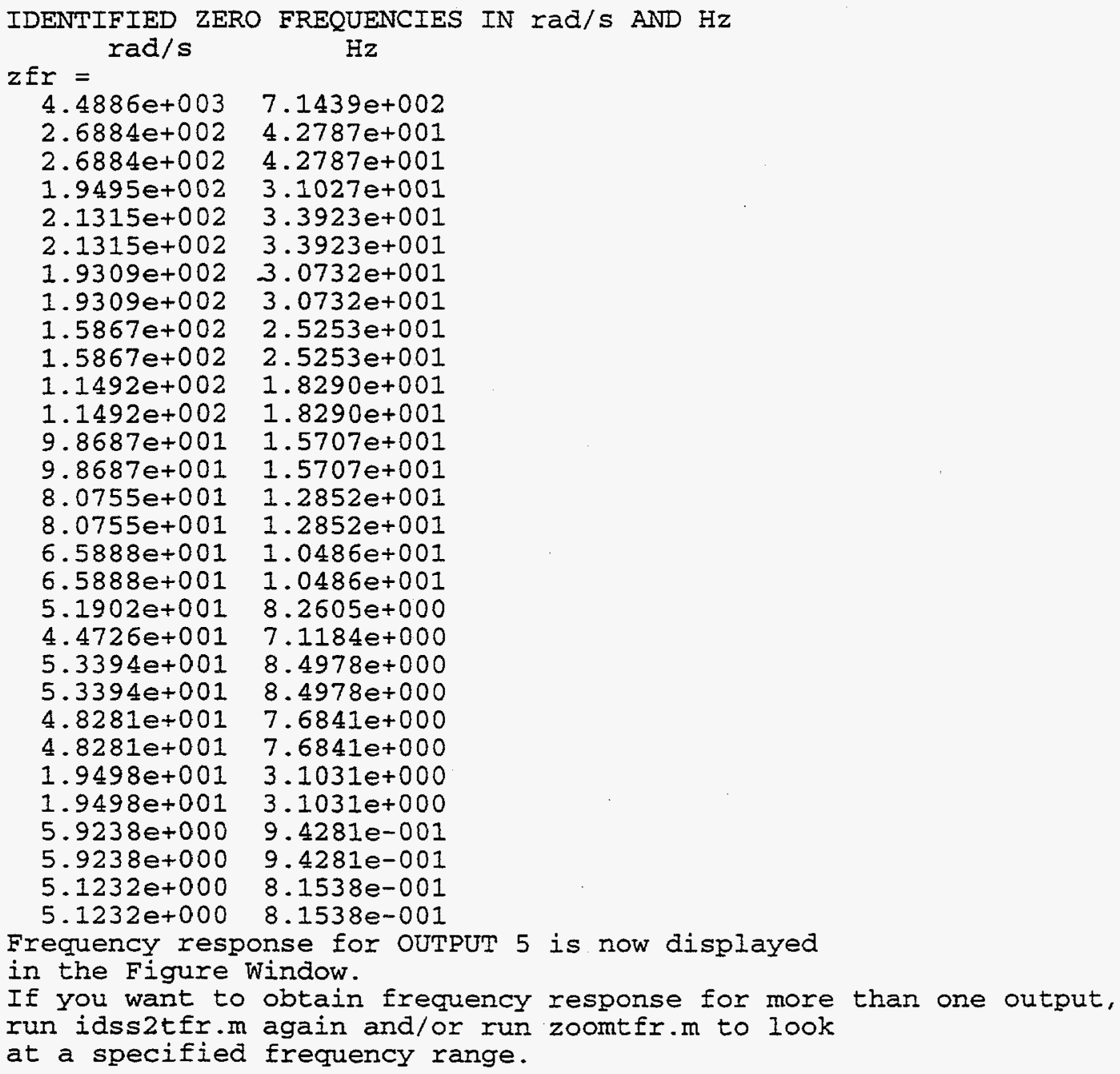



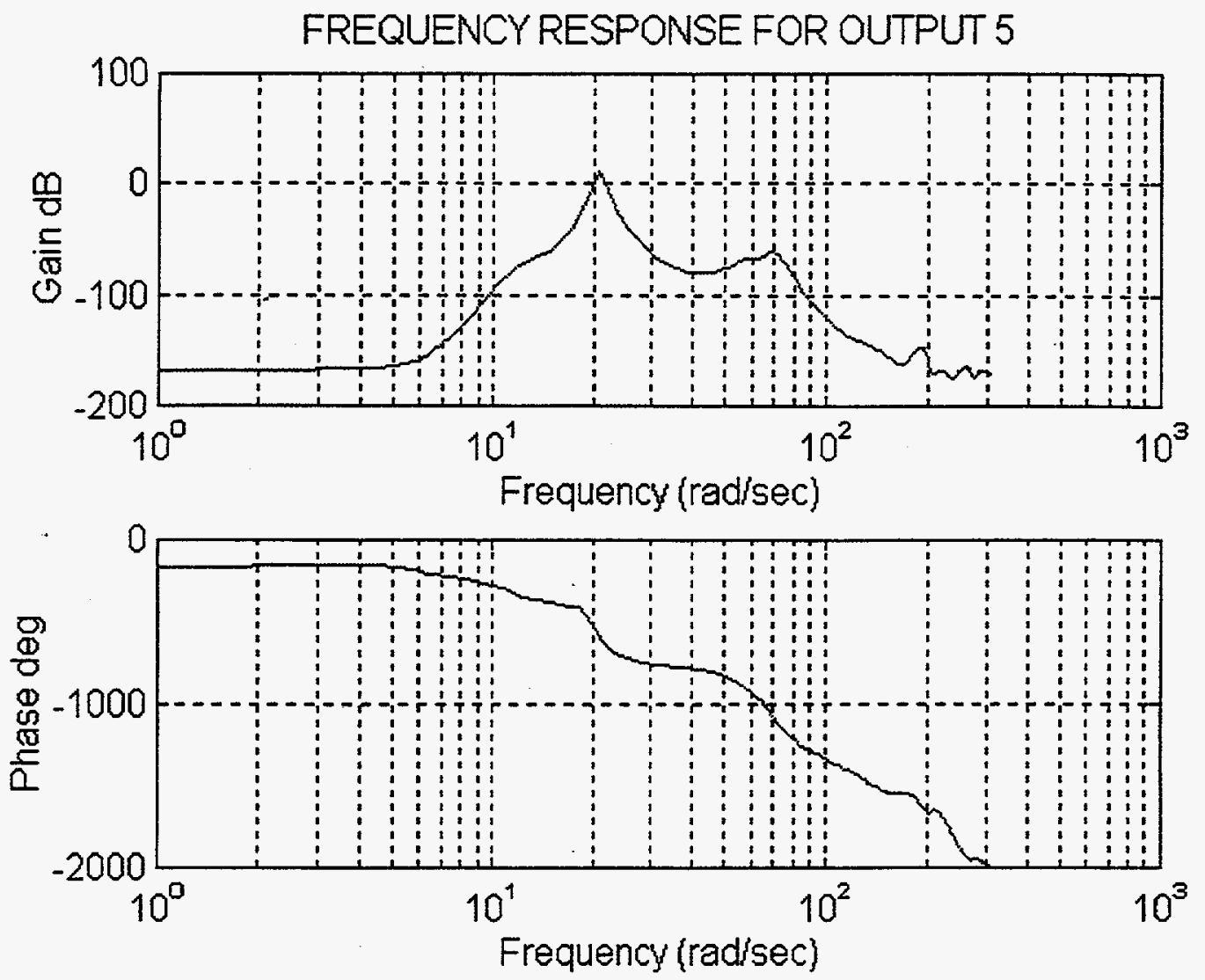

FREQUENCY RESPONSE FOR OUTPUT 5
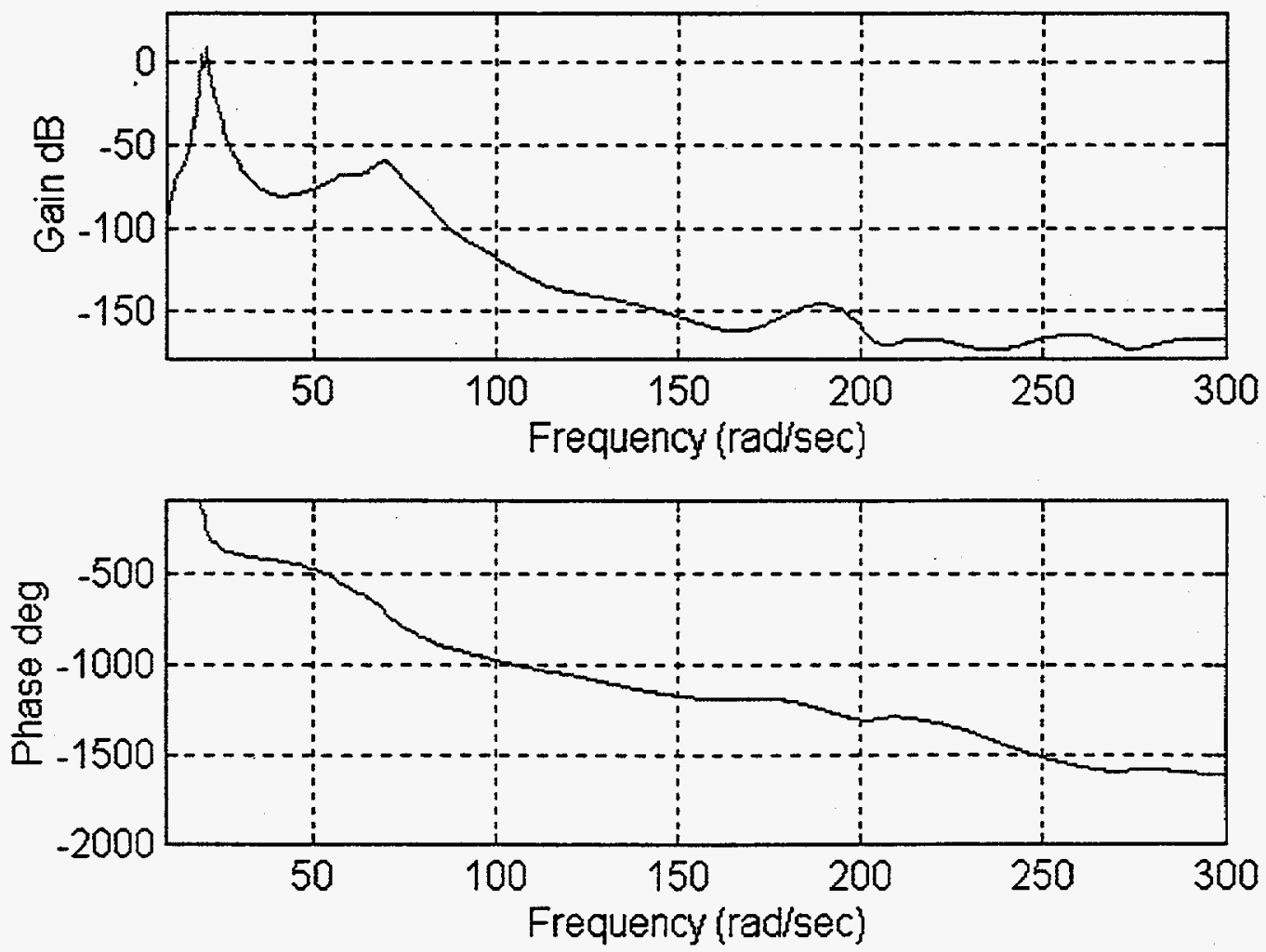


\section{Identification Procedure and Results for the Data File ib10104r.dat}

\section{Identification of Observer/Kalman Filter Model}

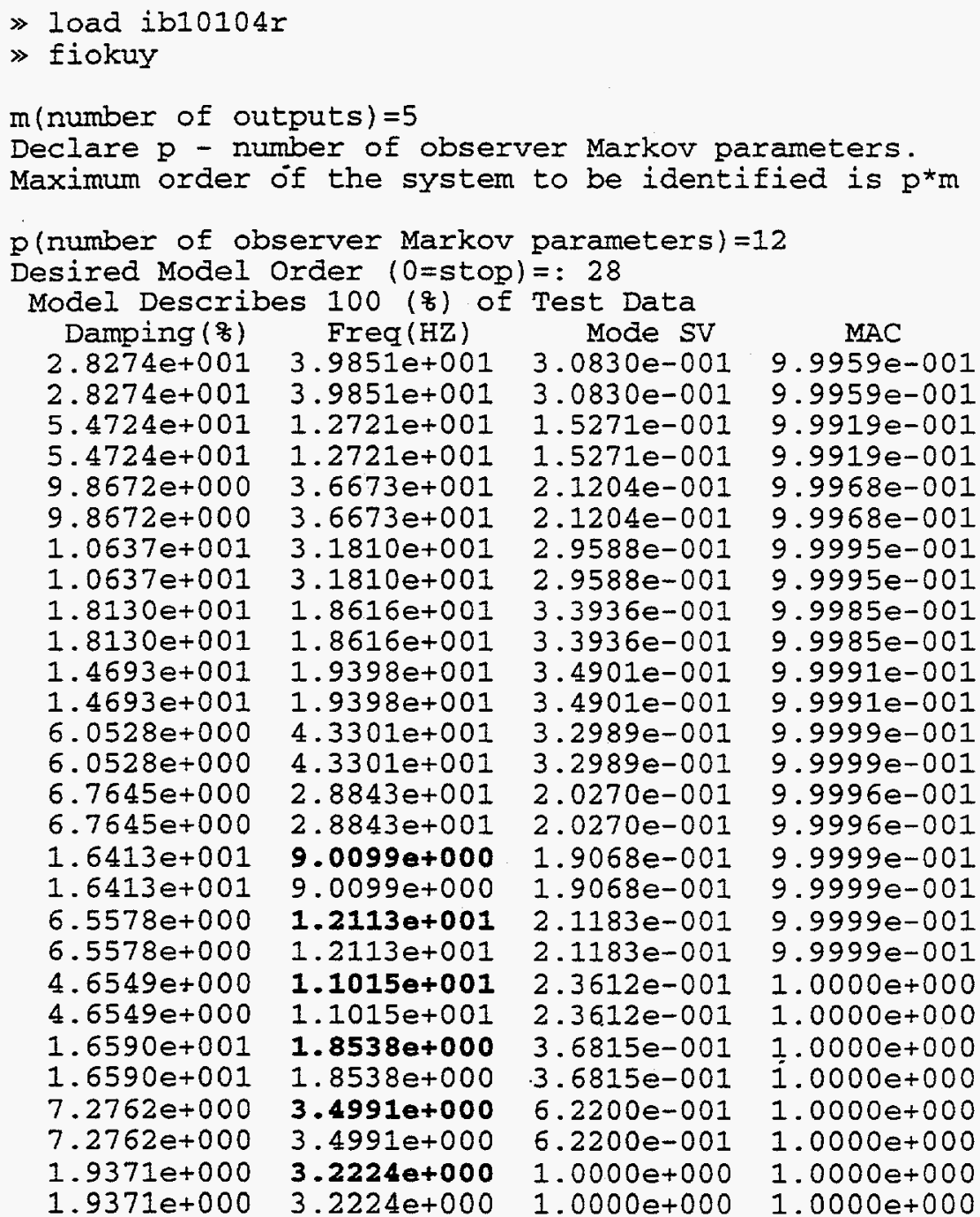

ENTER to continue.

Identification error is now displayed in the Figure window Identified system matrices are available as Af, Bf, Cf, Df observer gain matrix is available as $G$ 


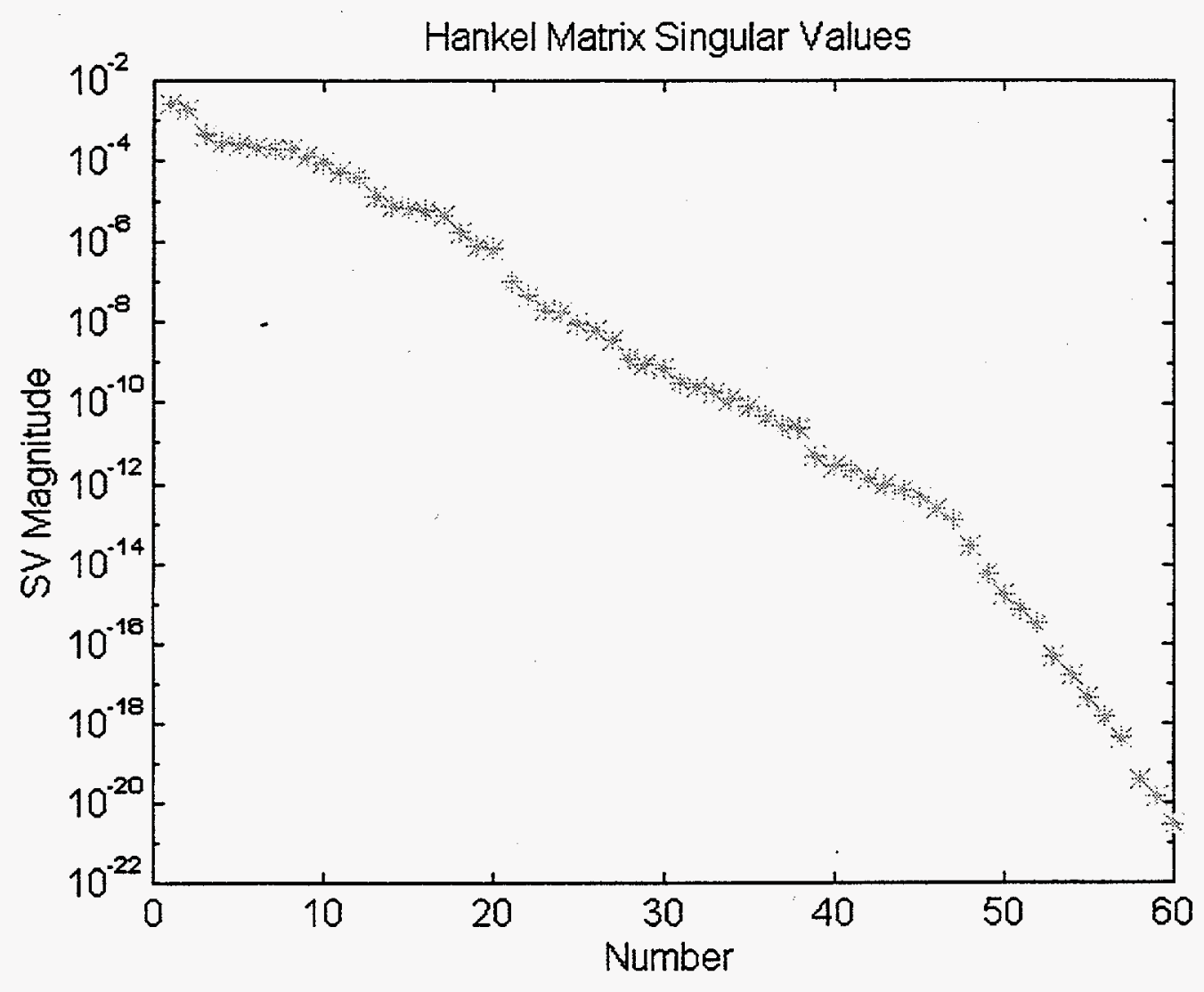

Identification Error

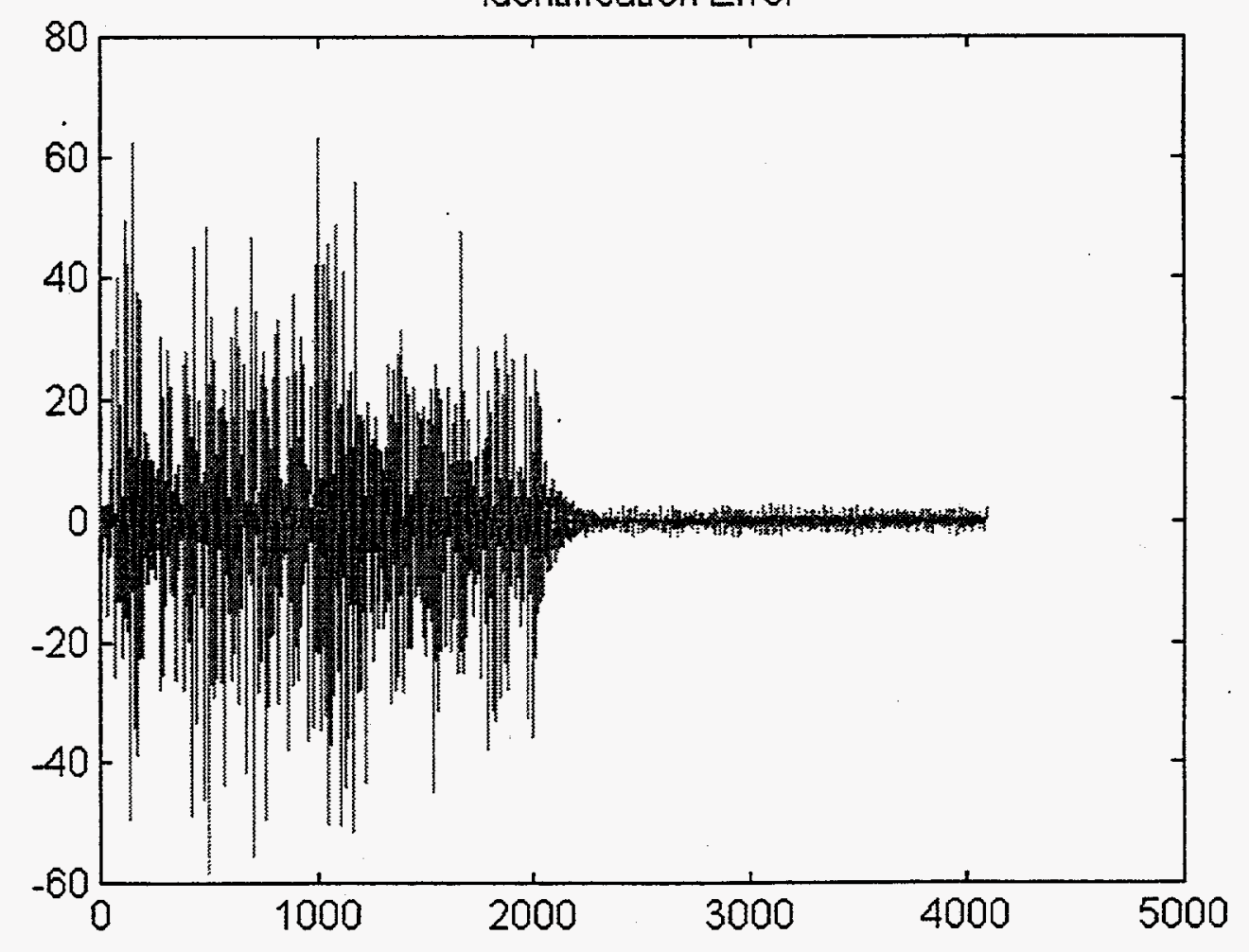




\title{
Frequency Response for OUTPUT1
}

\author{
$\gg$ idss2tfr
}

onr (output $n r$ for freq. resp) $=1$ IDENTIFIED SYSTEM EIGENVALUES

\section{$r 1=$}

$-1.6468 e+001+2.7157 e+002 i$

$-1.6468 e+001-2.7157 e+002 i$

$-7.0797 e+001+2.4017 e+002 i$

$-7.0797 e+001-2.4017 e+002 i$

$-2.2736 e+001+2.2930 e+002 i$

$-2.2736 e+001-2.2930 e+002 i$

$-2.1261 e+001+1.9874 e+002 i$

$-2.1261 e+001-1.9874 e+002 i$

$-1.2259 e+001+1.8081 e+002 i$

$-1.2259 e+001-1.8081 e+002 i$

$-1.7908 e+001+1.2056 e+002 i$

$-1.7908 e+001-1.2056 e+002 i$

$-2.1207 e+001+1.1503 e+002 i$

$-2.1207 e+001-1.1503 e+002 i$

$-4.3739 e+001+6.6898 e+001 i$

$-4.3739 e+001-6.6898 e+001 i$

$-4.9911 e+000+7.5946 e+001 i$

$-4.9911 e+000-7.5946 e+001 i$

$-3.2215 e+000+6.9132 e+001 i$

$-3.2215 e+000-6.9132 e+001 i$

$-9.2913 e+000+5.5843 e+001 i$

$-9.2913 e+000-5.5843 e+001 i$

$-1.5997 e+000+2.1927 e+001 i$

$-1.5997 e+000-2.1927 e+001 i$

$-3.9220 e-001+2.0243 e+001 i$

$-3.9220 e-001-2.0243 e+001 i$

$-1.9324 e+000+1.1486 e+001 i$

$-1.9324 e+000-1.1486 e+001 i$ 


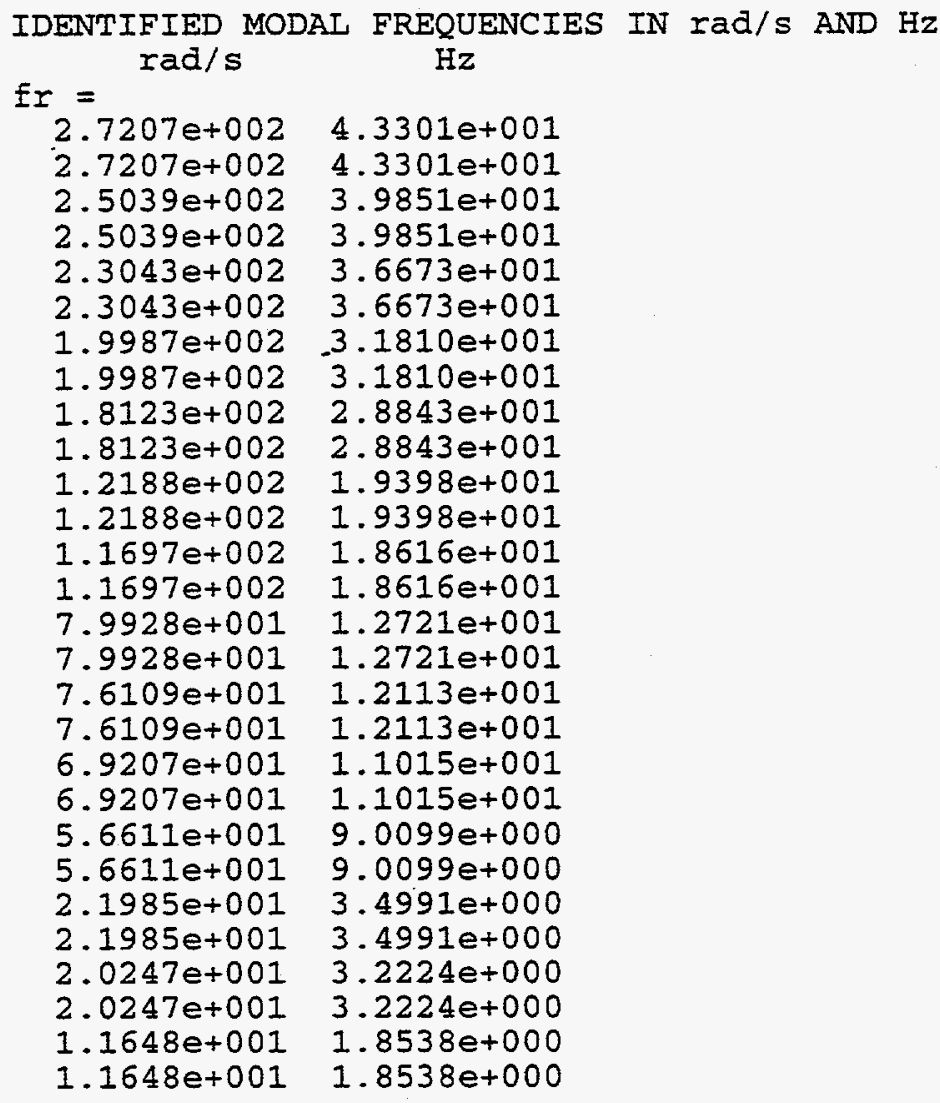


IDENTIFIED SYSTEM ZEROS RELATED TO THE OUTPUT 1

$\mathrm{ZER}=$

$2.4246 e+002+1.5013 e+002 i$

$2.4246 e+002-1.5013 e+002 i$

$-1.3965 e+001+2.6568 e+002 i$

$-1.3965 e+001-2.6568 e+002 i$

$-1.4512 e+001+2.3797 e+002 i$

$-1.4512 e+001-2.3797 e+002 i$

$-1.6336 e+001+1.9955 e+002 i$

$-1.6336 e+001-1.9955 e+002 i$

$-3.2130 e+001+1.6650 e+002 i$

$-3.2130 e+001-1.6650 e+002 i$

$-1.2605 e+002$

$9.4994 e+001$

$3.1697 e+001+1.1283 e+002 i$

$3.1697 e+001-1.1283 e+002 i$

$-1.0634 e+001+1.1823 e+002 i$

$-1.0634 e+001-1.1823 e+002 i$

$4.3638 e+001+5.4064 e+001 i$

$4.3638 e+001-5.4064 e+001 i$

$-2.3595 e+001+7.3714 e+001 i$

$-2.3595 e+001-7.3714 e+001 i$

$-5.7020 e+000+7.0914 e+001 i$

$-5.7020 e+000-7.0914 e+001 i$

$-3.6043 e+001+2.7636 e+001 i$

$-3.6043 e+001-2.7636 e+001 i$

$-7.2045 e-001+1.9744 e+001 i$

$-7.2045 e-001-1.9744 e+001 i$

$-2.3162 e+001$

$2.4051 e+000$

B -43 
IDENTIFIED ZERO FREQUENCIES IN rad/s AND Hz zfr $=$

$2.8518 e+002 \quad 4.5388 e+001$

$2.8518 e+002 \quad 4.5388 e+001$

$2.6604 e+002 \quad 4.2342 e+001$

$2.6604 e+002 \quad 4.2342 e+001$

$2.3842 e+002 \quad 3.7945 e+001$

$2.3842 e+002 \quad 3.7945 e+001$

$2.0022 e+002 \quad 3.1866 e+001$

$2.0022 e+002 \quad 3.1866 e+001$

$1.6957 e+002 \quad 2.6988 e+001$

$1.6957 e+002 \quad 2.6988 e+001$

$1.2605 e+002 \quad 2.0061 e+001$

$9.4994 \mathrm{e}+001 \quad 1.5119 \mathrm{e}+001$

$1.1720 e+002 \quad 1.8653 e+001$

$1.1720 e+002 \quad 1.8653 e+001$

$1.1871 e+002 \quad 1.8893 e+001$

$1.1871 \mathrm{e}+002 \quad 1.8893 \mathrm{e}+001$

$6.9478 e+001 \quad 1.1058 e+001$

$6.9478 e+001 \quad 1.1058 e+001$

$7.7398 e+001 \quad 1.2318 e+001$

$7.7398 e+001 \quad 1.2318 e+001$

$7.1143 e+001 \quad 1.1323 e+001$

$7.1143 e+001 \quad 1.1323 e+001$

$4.5419 e+001 \quad 7.2286 e+000$

$4.5419 e+001 \quad 7.2286 e+000$

$1.9757 e+001 \quad 3.1444 e+000$

$1.9757 e+001 \quad 3.1444 e+000$

$2.3162 e+001 \quad 3.6864 e+000$

$2.4051 e+000 \quad 3.8279 e-001$

Frequency response for OUTPUT 1 is now displayed in the Figure Window.

If you want to obtain frequency response for more than one output, run idss2tfr.m again and/or run zoomtfr.m to look at a specified frequency range. 

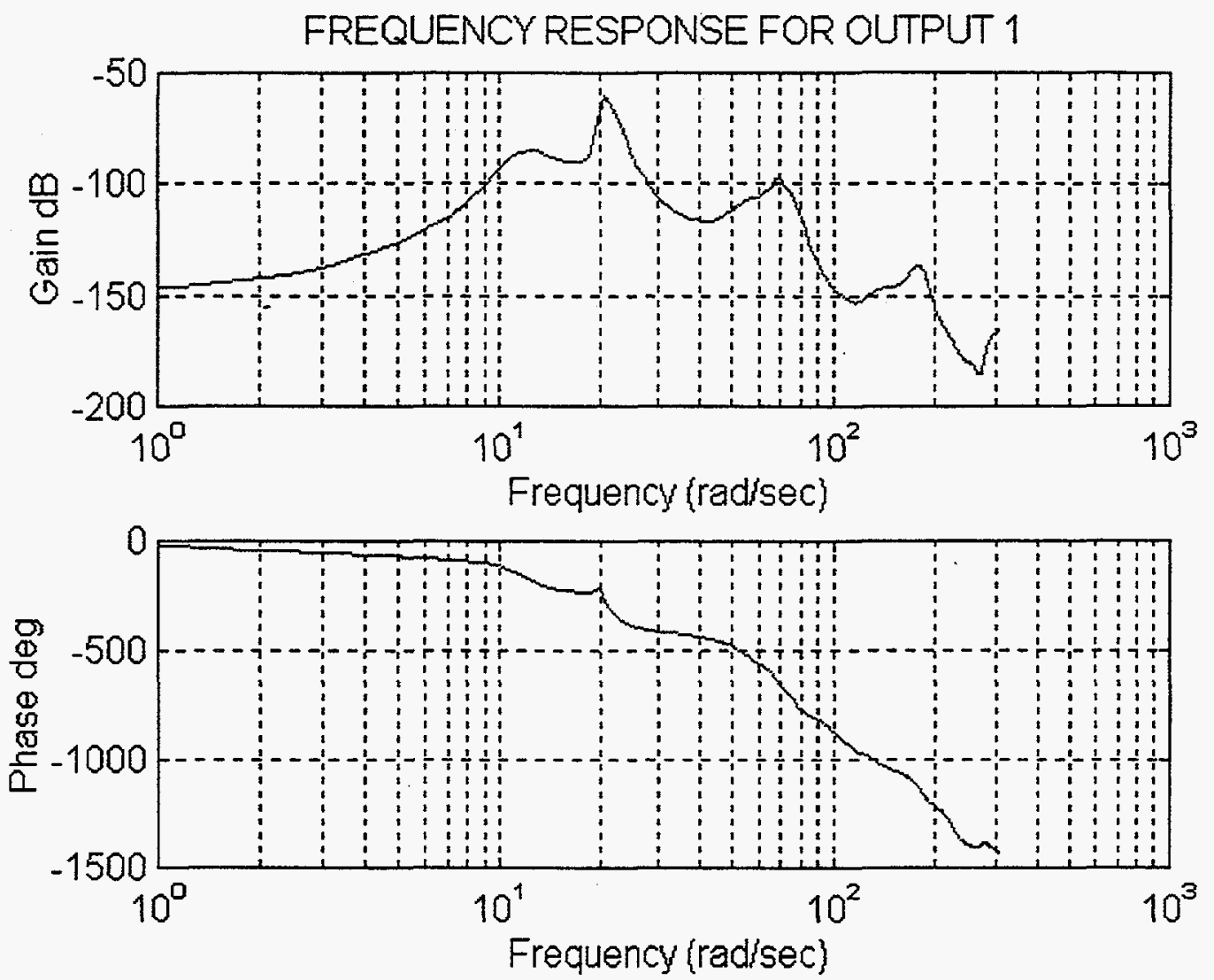

FREQUENCY RESPONSE FOR OUTPUT 1
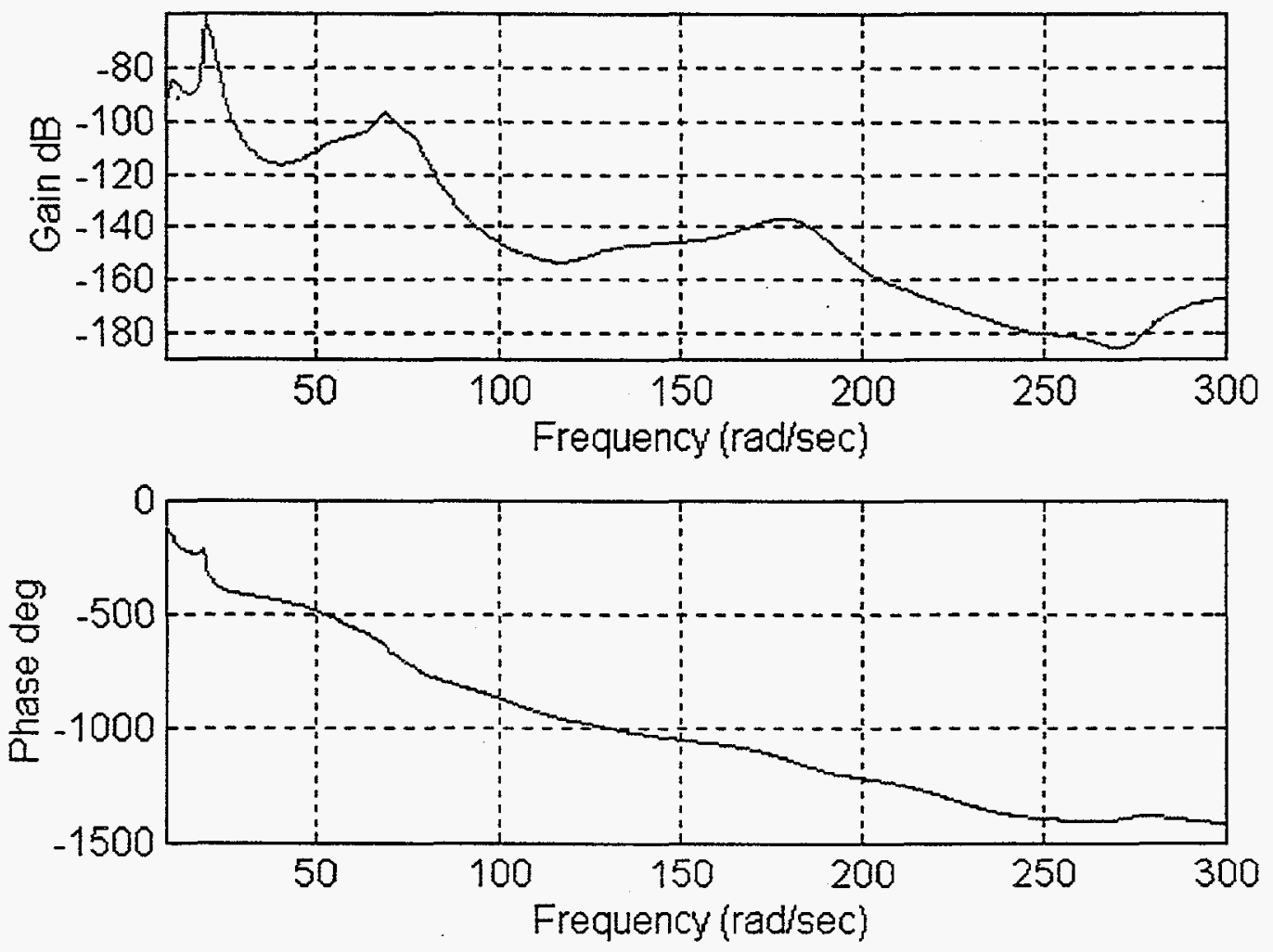
Frequency Response for OUTPUT2

$\gg$ idss $2 t f r$

onr (output nr for freq. resp) $=2$

IDENTIFIED SYSTEM ZEROS RELAATED TO THE OUTPUT 2

$\mathrm{ZER}=$

$5.8626 e+002$

$2.8096 \mathrm{e}+002$

$-1.2240 e+001+2.6729 e+002 i$

$-1.2240 e+001-2.6729 e+002 i$

$-1.2796 e+001+2.3676 e+002 i$

$-1.2796 e+001-2.3676 e+002 i$

$-2.0370 e+001+2.0042 e+002 i$

$-2.0370 e+001-2.0042 e+002 i$

$-2.4428 e+001+1.7326 e+002 i$

$-2.4428 e+001-1.7326 e+002 i$

$2.6572 e+001+1.1731 e+002 i$

$2.6572 e+001-1.1731 e+002 i$

$-8.8423 e+000+1.1576 e+002 i$

$-8.8423 e+000-1.1576 e+002 i$

$-3.1948 e+001+8.1237 e+001 i$

$-3.1948 e+001-8.1237 e+001 i$

$3.3395 e+001+5.1245 e+001 i$

$3.3395 e+001-5.1245 e+001 i$

$-1.4412 e+001+7.7213 e+001 i$

$-1.4412 e+001-7.7213 e+001 i$

$-6.0489 e+000+7.0305 e+001 i$

$-6.0489 e+000-7.0305 e+001 i$

$-3.2377 e+001+3.4845 e+001 i$

$-3.2377 e+001-3.4845 e+001 i$

$-2.6167 e+001$

$-7.2046 e-001+1.9601 e+001 i$

$-7.2046 e-001-1.9601 e+001 i$

$2.6196 e+000$ 
IDENTIFIED ZERO FREQUENCIES IN rad/s AND Hz $\mathrm{rad} / \mathrm{s}$ $\mathrm{zfr}=$

$\mathrm{Hz}$

$5.8626 e+002$

$2.8096 e+002$

$9.3307 e+001$

4. $4715 e+001$

$2.6757 e+002$

$2.6757 e+002$

4. $2585 e+001$

$2.3711 e+002$

$4.2585 e+001$

2. 3711 e+002

$2.0145 e+002$

$3.7737 e+001$

$3.7737 e+001$

$2.0145 e+002$

$1.7497 e+002$

$1.7497 e+002$

$-3.2062 e+001$

3. $2062 \mathrm{e}+001$

$2.7847 e+001$

1. $2028 e+002$

$2.7847 e+001$

$1.2028 \mathrm{e}+002$

$1.9144 e+001$

$1.1610 e+002$

1. $9144 \mathrm{e}+001$

1. 1610 e +002

$1.8478 e+001$

$8.7293 e+001$

1. $8478 e+001$

$8.7293 e+001$

$1.3893 e+001$

$6.1166 e+001$

1. $3893 e+001$

$6.1166 e+001$

$9.7349 e+000$

$7.8546 e+001$

$9.7349 e+000$

$7.8546 e+001$

1. $2501 e+001$

$7.0564 \mathrm{e}+001$

1. $2501 e+001$

$7.0564 e+001$

$1.1231 e+001$

$4.7565 e+001$

1. 1231 e +001

$4.7565 e+001$

$7.5702 e+000$

$2.6167 e+001$

$7.5702 e+000$

$1.9614 e+001$

4. $1646 \mathrm{e}+000$

$1.9614 e+001$

$3.1217 e+000$

$2.6196 e+000$

3. $1217 e+000$

$4.1693 e-001$

Frequency response for OUTPUT 2 is now displayed

in the Figure Window.

If you want to obtain frequency response for more than one output, run idss2tfr.m again and/or run zoomtfr.m to look at a specified frequency range. 

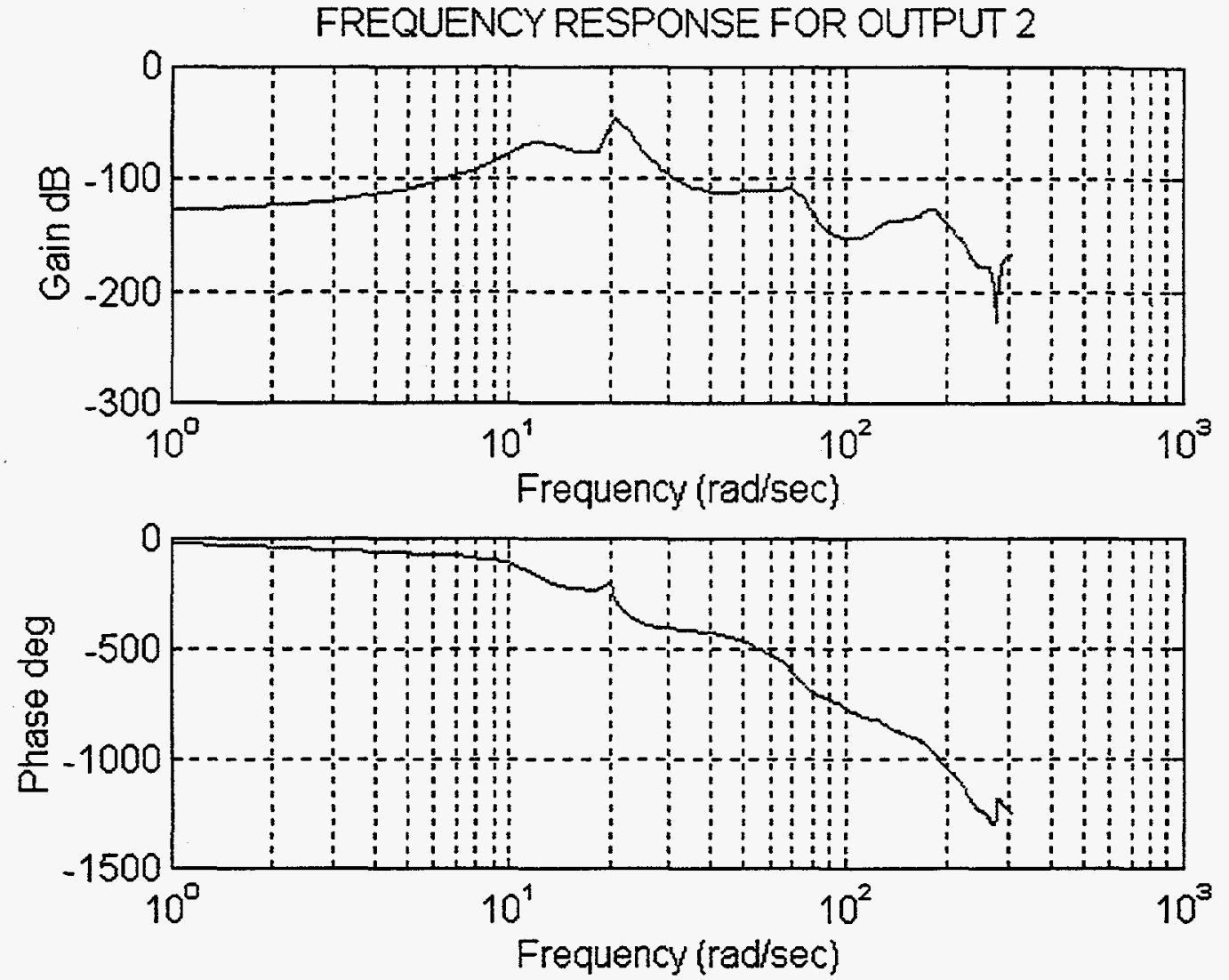

FREQUENCY RESPONSE FOR OUTPUT 2
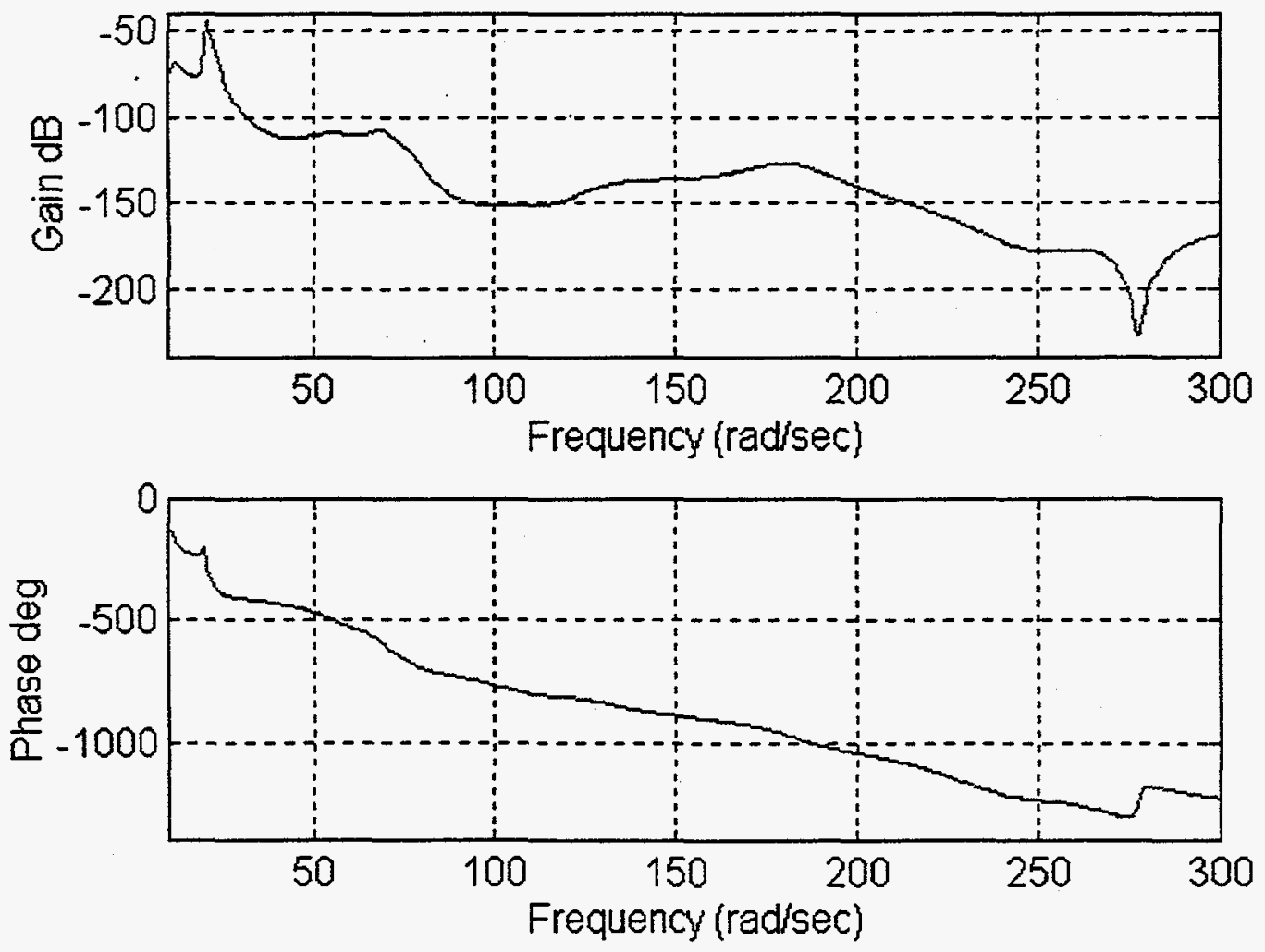


\section{Frequency Response for OUTPUT3 (Collocation Case)}

$\gg$ idss2tfr

onr (output $\mathrm{nr}$ for freq. resp) $=3$

IDENTIFIED SYSTEM ZEROS RELATED TO THE OUTPUT 3 $\mathrm{ZER}=$

$-1.3735 e+003$

$3.5136 e+001+2.8735 e+002 i$

$3.5136 e+001-2.8735 e+002 i$

$2.2829 e-001+2.3573 e+002 i$

$2.2829 e-001-2.3573 e+002 i$

$-2.5213 e+001+2.1863 e+002 i$

$-2.5213 e+001-2.1863 e+002 i$

$-1.6651 e+001+1.7706 e+002 i$

$-1.6651 e+001-1.7706 e+002 i$

$-3.0199 e+001+1.4232 e+002 i$

$-3.0199 e+001-1.4232 e+002 i$

$-6.9928 e+000+1.0784 e+002 i$

$-6.9928 e+000-1.0784 e+002 i$

$2.2280 e+001+8.7760 e+001 i$

$2.2280 e+001-8.7760 e+001 i$

$-1.4852 e+001+7.1257 e+001 i$

$-1.4852 e+001-7.1257 e+001 i$

$-5.6281 e+000+7.0977 e+001 i$

$-5.6281 e+000-7.0977 e+001 i$

$-3.8826 e+001+2.3718 e+001 i$

$-3.8826 e+001-2.3718 e+001 i$

$-7.5892 e+000+4.9831 e+001 i$

$-7.5892 e+000-4.9831 e+001 i$

$-7.6892 e-001+2.0387 e+001 i$

$-7.6892 e-001-2.0387 e+001 i$

$-5.8701 e-001+1.0888 e+001 i$

$-5.8701 e-001-1.0888 e+001 i$

$4.0005 \mathrm{e}+000$ 


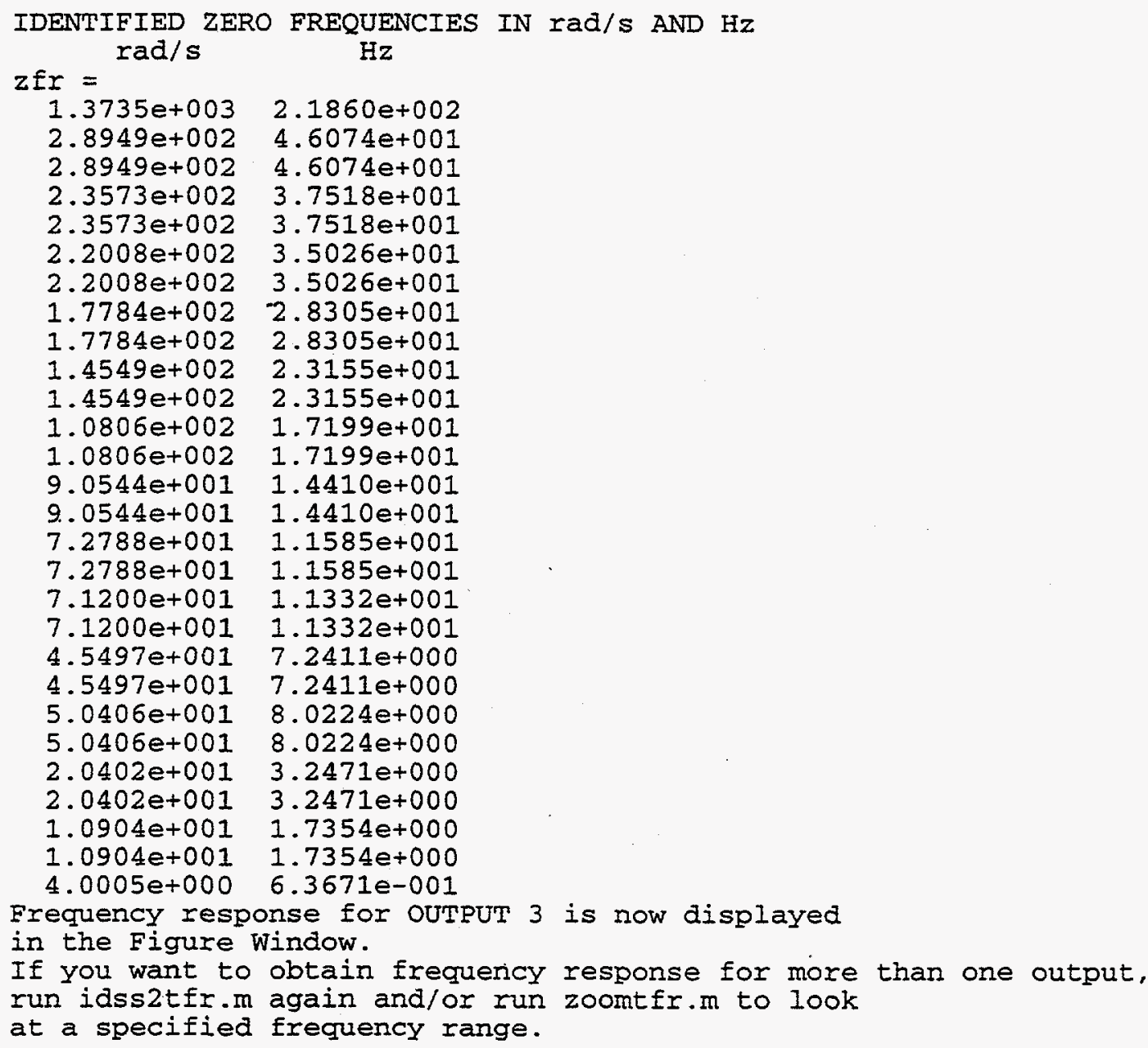



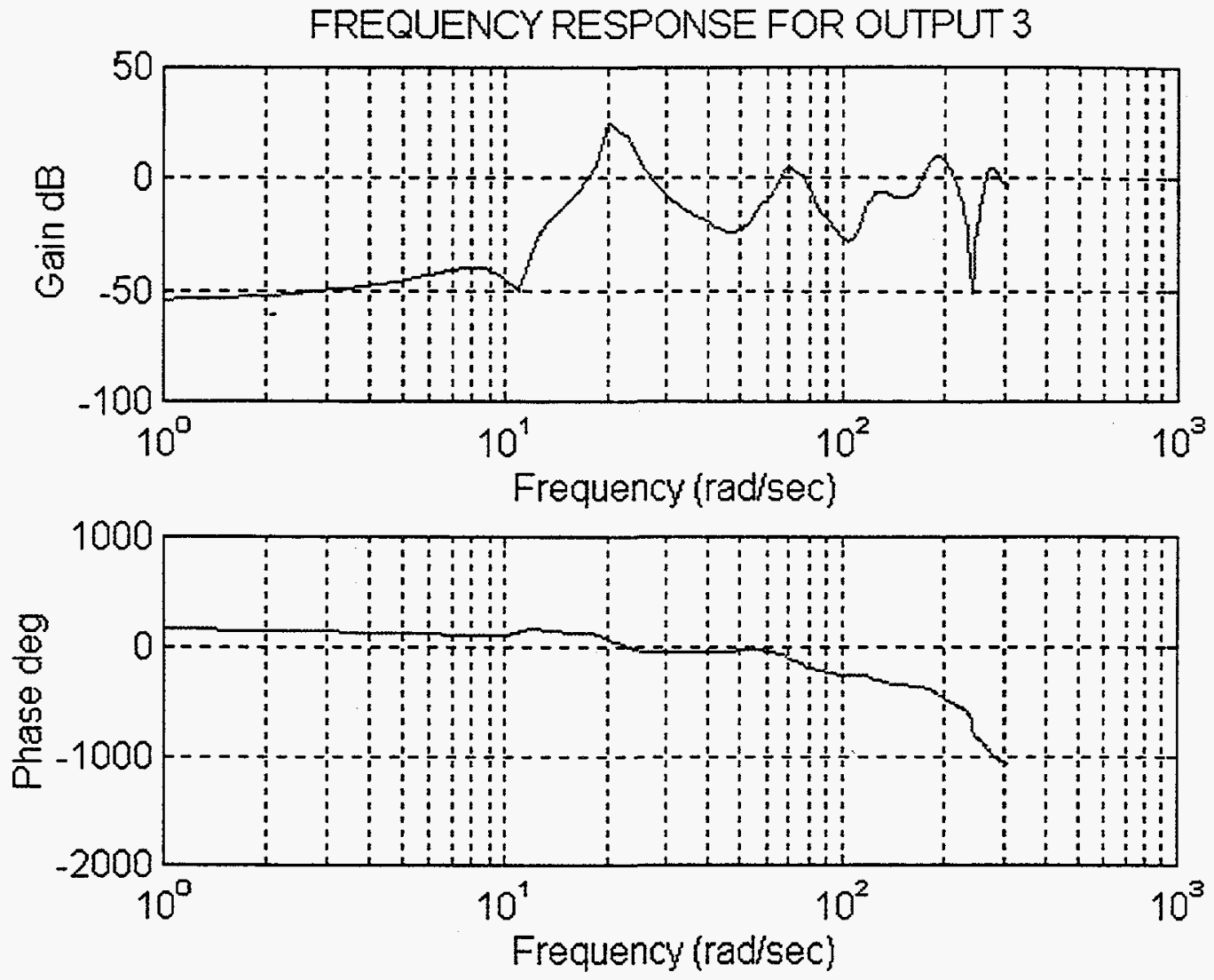

FREQUENCY RESPONSE FOR OUTPUT 3
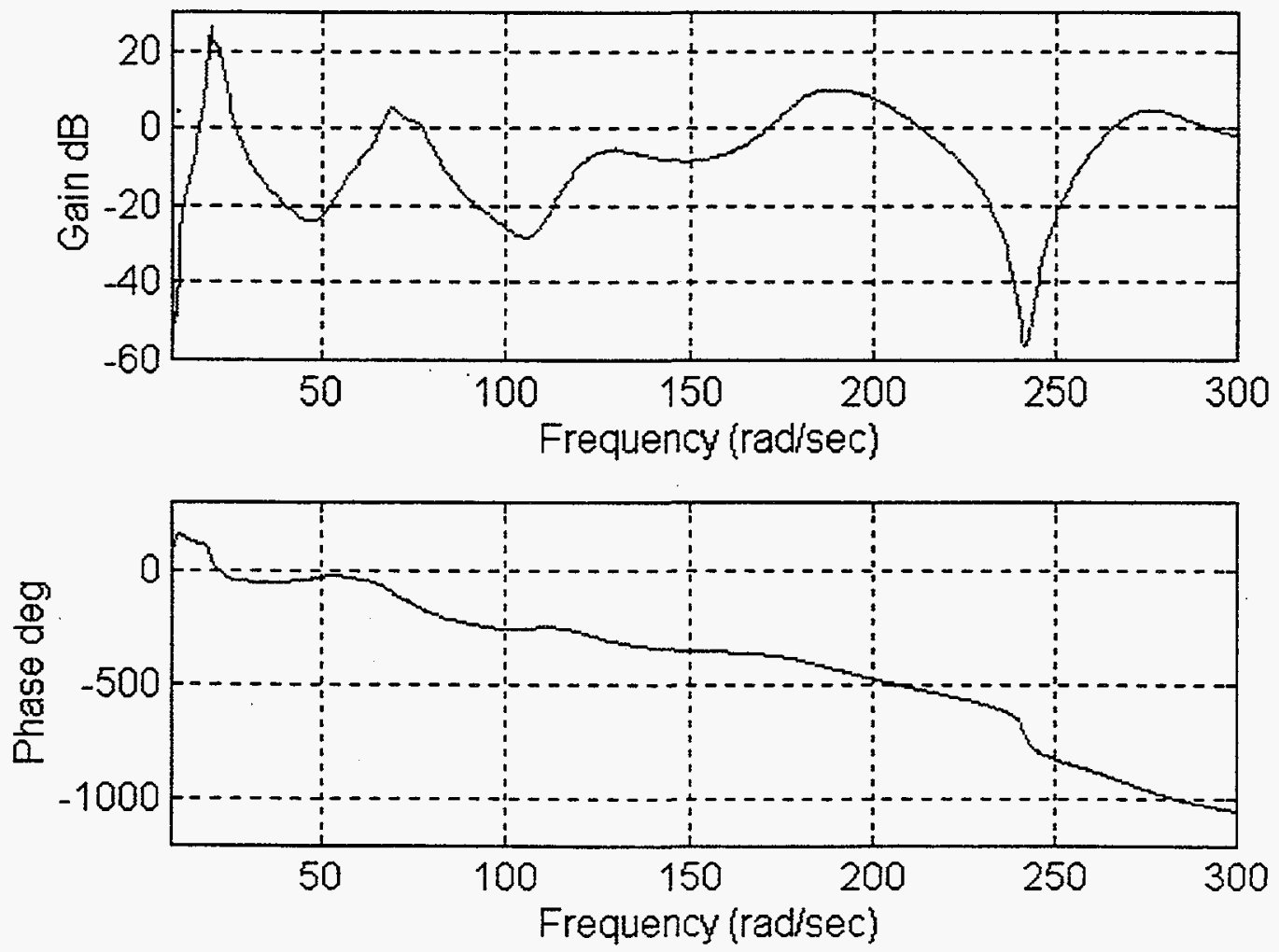
Frequency Response for OUTPUT4

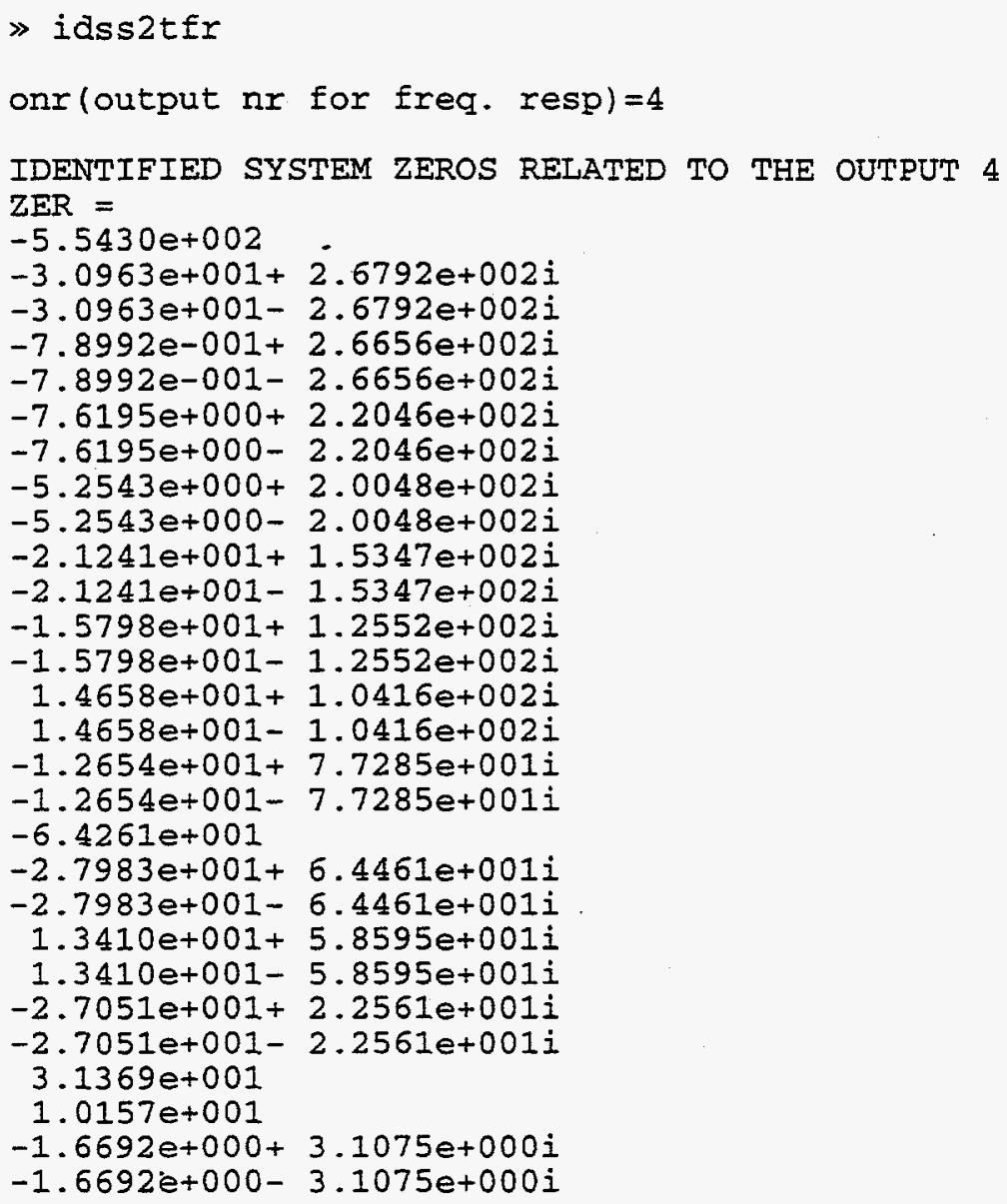




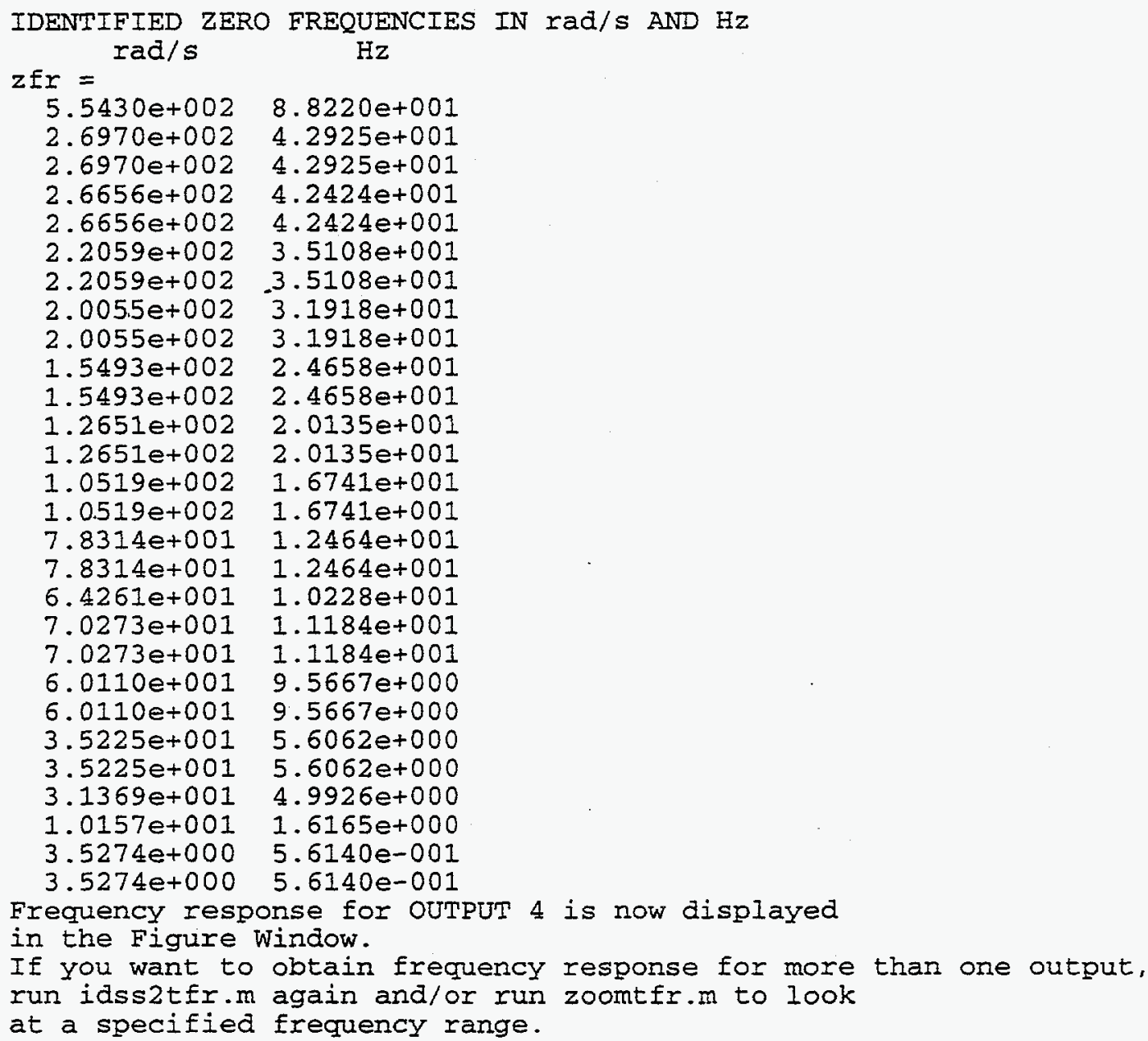



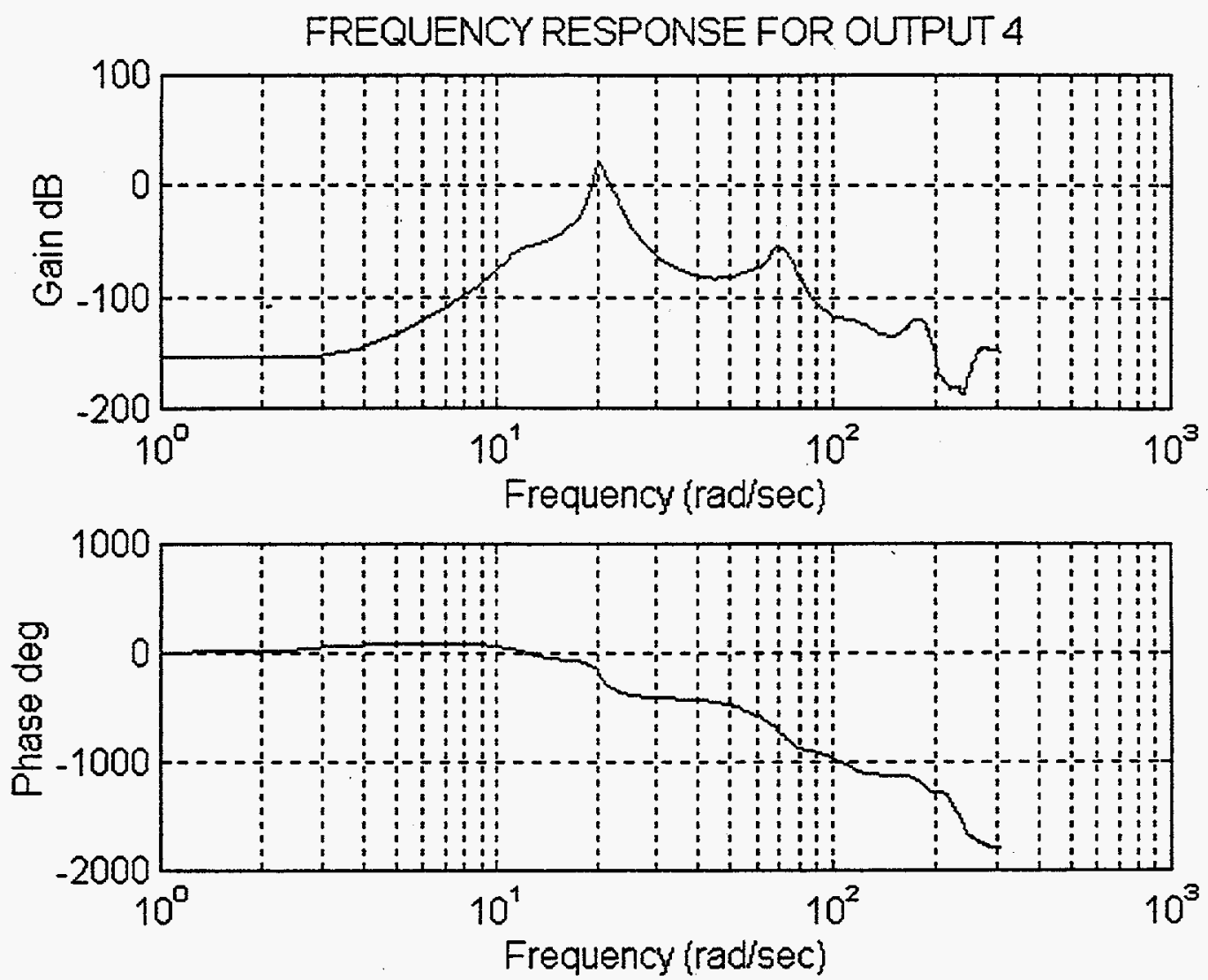

FREQUENCY RESPONSE FOR OUTPUT 4
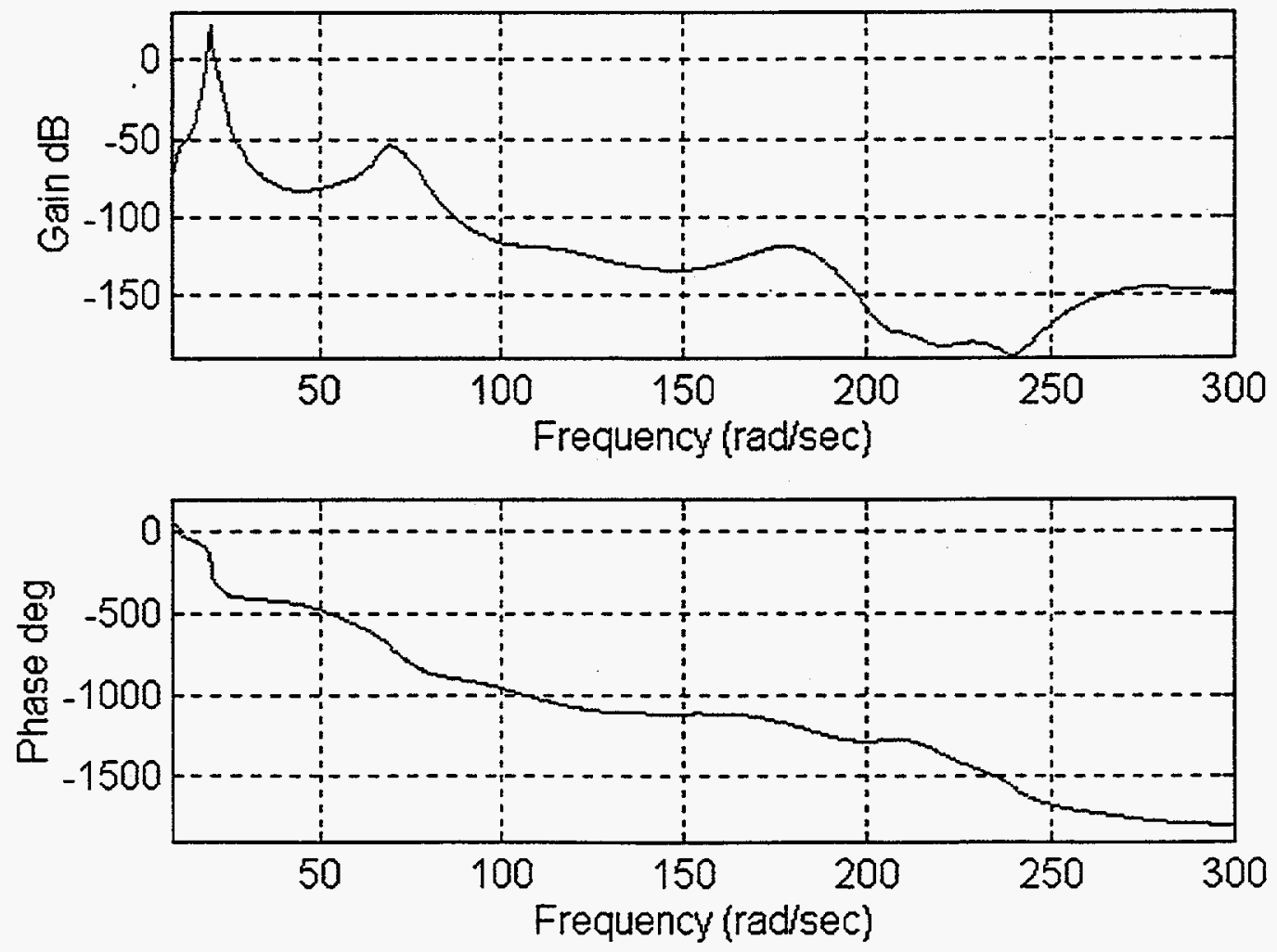


\section{Frequency Response for OUTPUT5}

$\gg$ idss 2 tfr

onr (output $\mathrm{nr}$ for freq. resp) $=5$

IDENTIFIED SYSTEM ZEROS RELATED TO THE OUTPUT 5 $\mathrm{ZER}=$

$-4.2731 e+001+2.9893 e+002 i$

$-4.2731 e+001-2.9893 e+002 i$

$3.5582 e+000+2.8259 e+002 i$

$3.5582 e+000-2.8259 e+002 i$

$-1.3021 e+001+2.2344 e+002 i$

$-1.3021 e+001-2.2344 e+002 i$

$-1.0887 e+002+1.6847 e+002 i$

$-1.0887 e+002-1.6847 e+002 i$

$-7.2381 e+000+2.0241 e+002 i$

$-7.2381 e+000-2.0241 e+002 i$

$-2.8261 e+001+1.4654 e+002 i$

$-2.8261 e+001-1.4654 e+002 i$

$-2.2934 e+001+1.2796 e+002 i$

$-2.2934 e+001-1.2796 e+002 i$

$1.3686 e+001+1.0591 e+002 i$

$1.3686 e+001-1.0591 e+002 i$

$-1.3177 e+001+7.6783 e+001 i$

$-1.3177 e+001-7.6783 e+001 i$

$-2.5733 e+001+6.1505 e+001 i$

$-2.5733 e+001-6.1505 e+001 i$

$1.3337 e+001+5.8709 e+001 i$

$1.3337 e+001-5.8709 e+001 i$

$3.3452 e+001$

$-2.1953 e+001+2.0608 e+001 i$

$-2.1953 e+001-2.0608 e+001 i$

$9.5994 e+000$

$-1.3696 e+000+3.1705 e+000 i$

$-1.3696 e+000-3.1705 e+000 i$ 


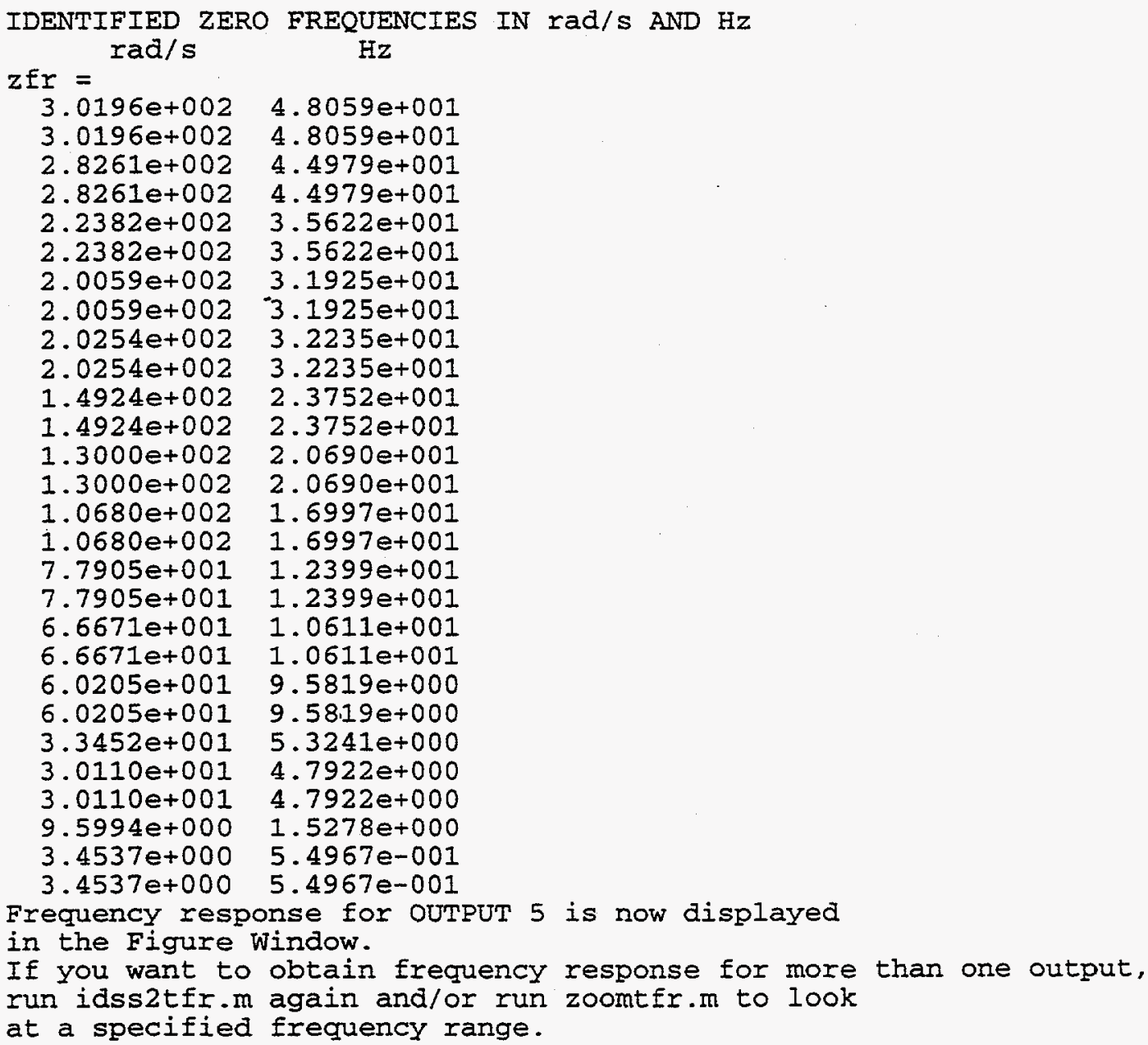



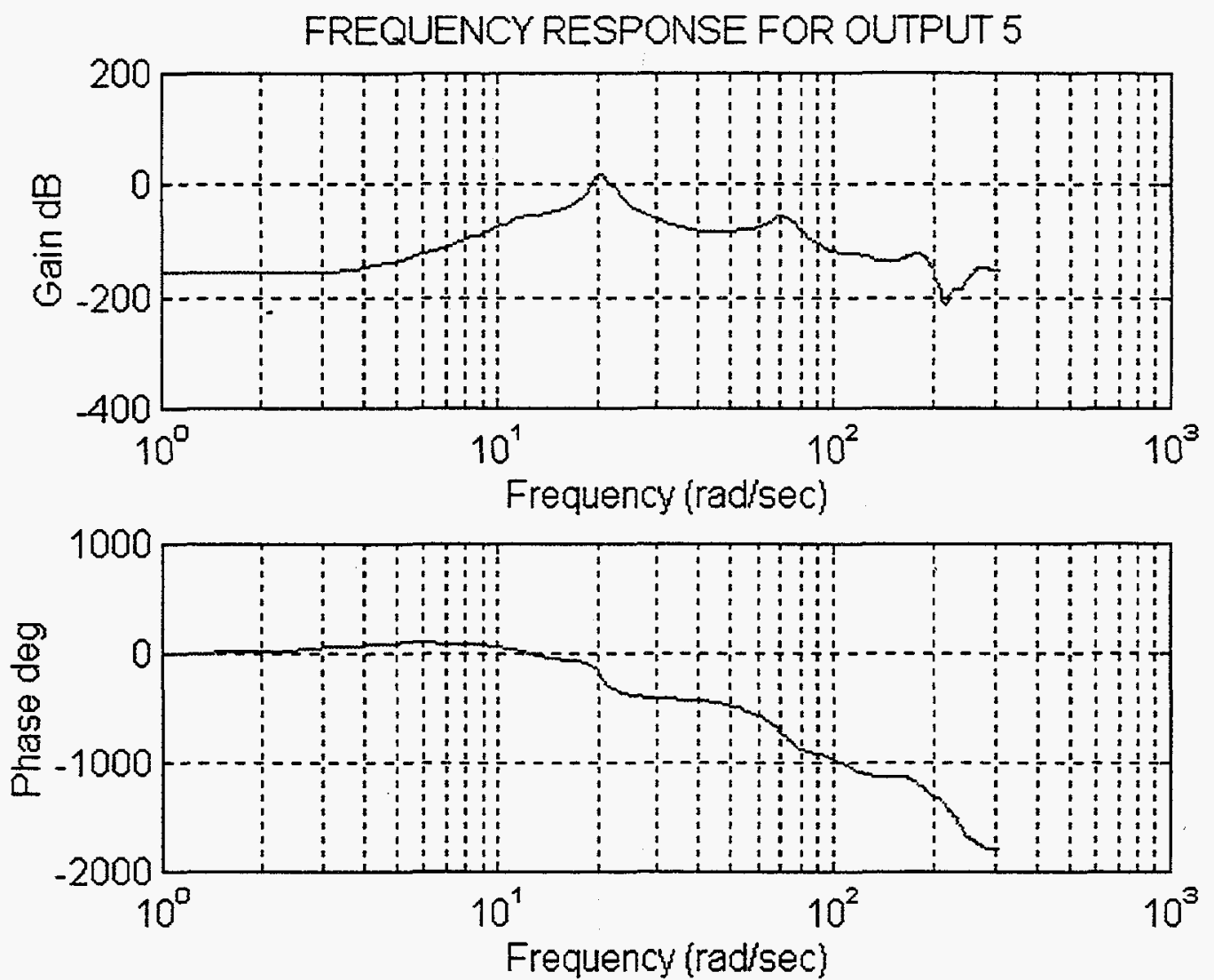

FREQUENCY RESPONSE FOR OUTPUT 5
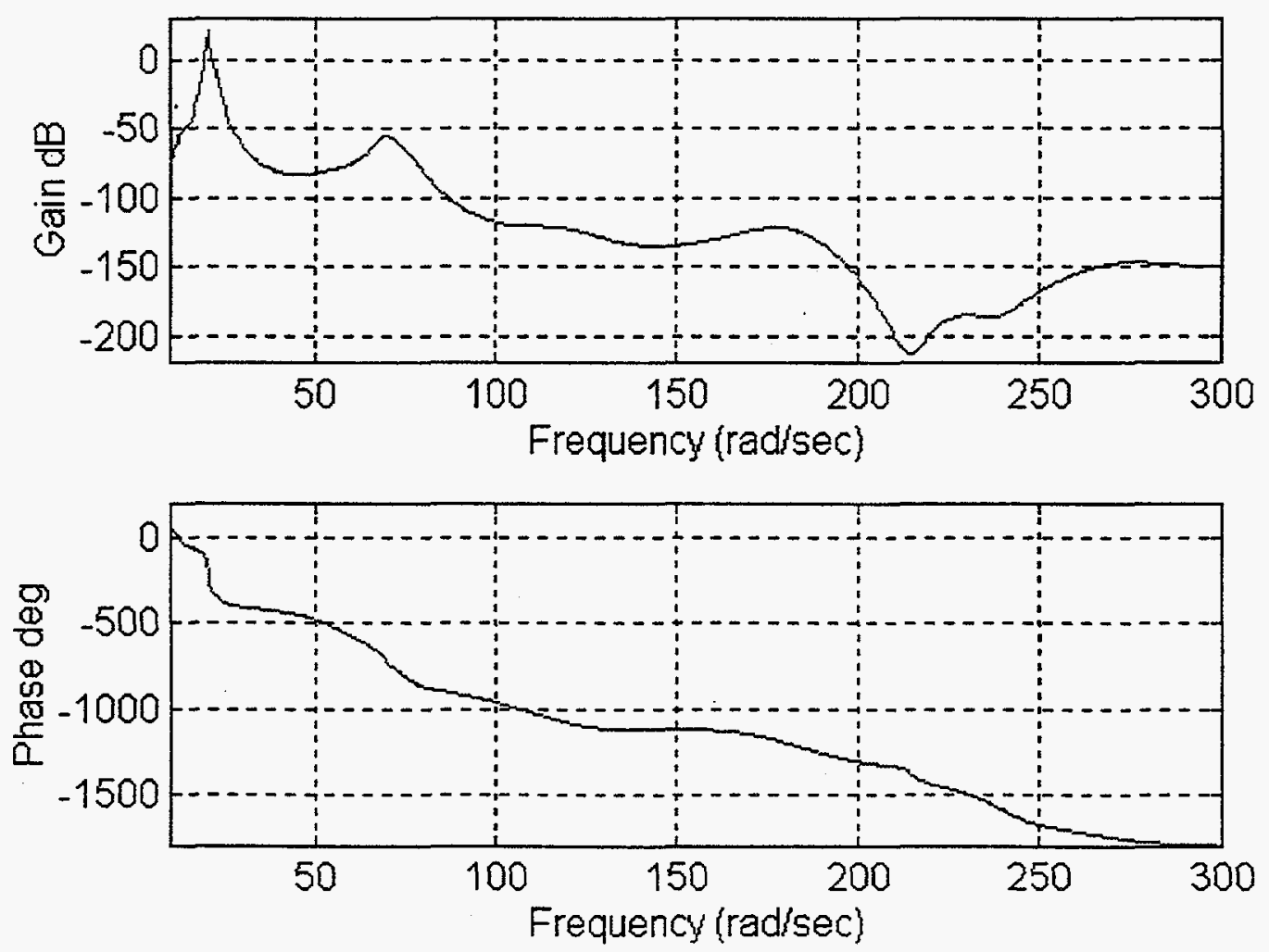


\section{Identification Procedure and Results for the Data File its0104r.dat}

\section{Identification of Observer/Kalman Filter Model}

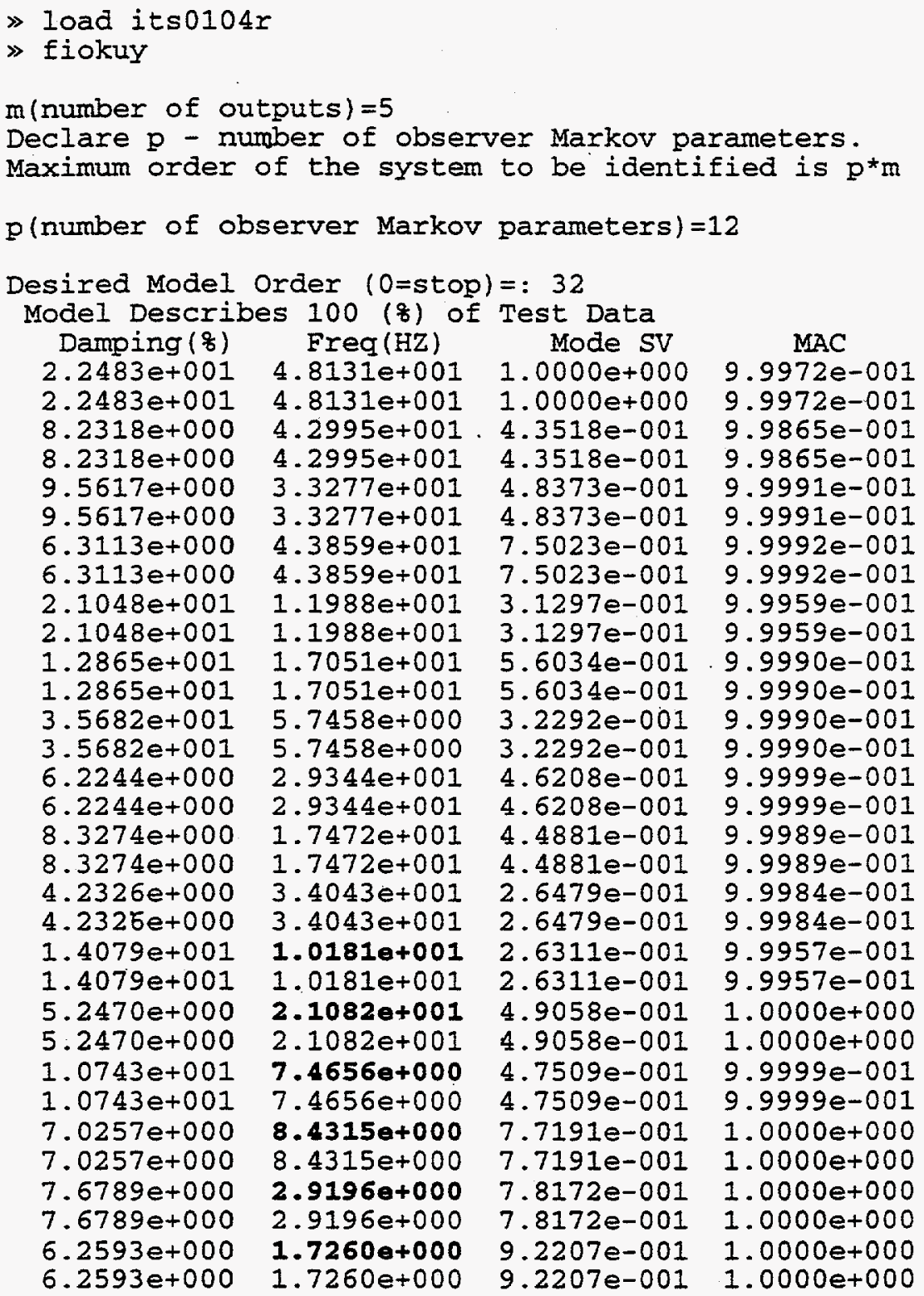

ENTER to continue.

Identification error is now displayed in the Figure window Identified system matrices are available as $A f, B f, C f, D f$ observer gain matrix is available as $G$ 

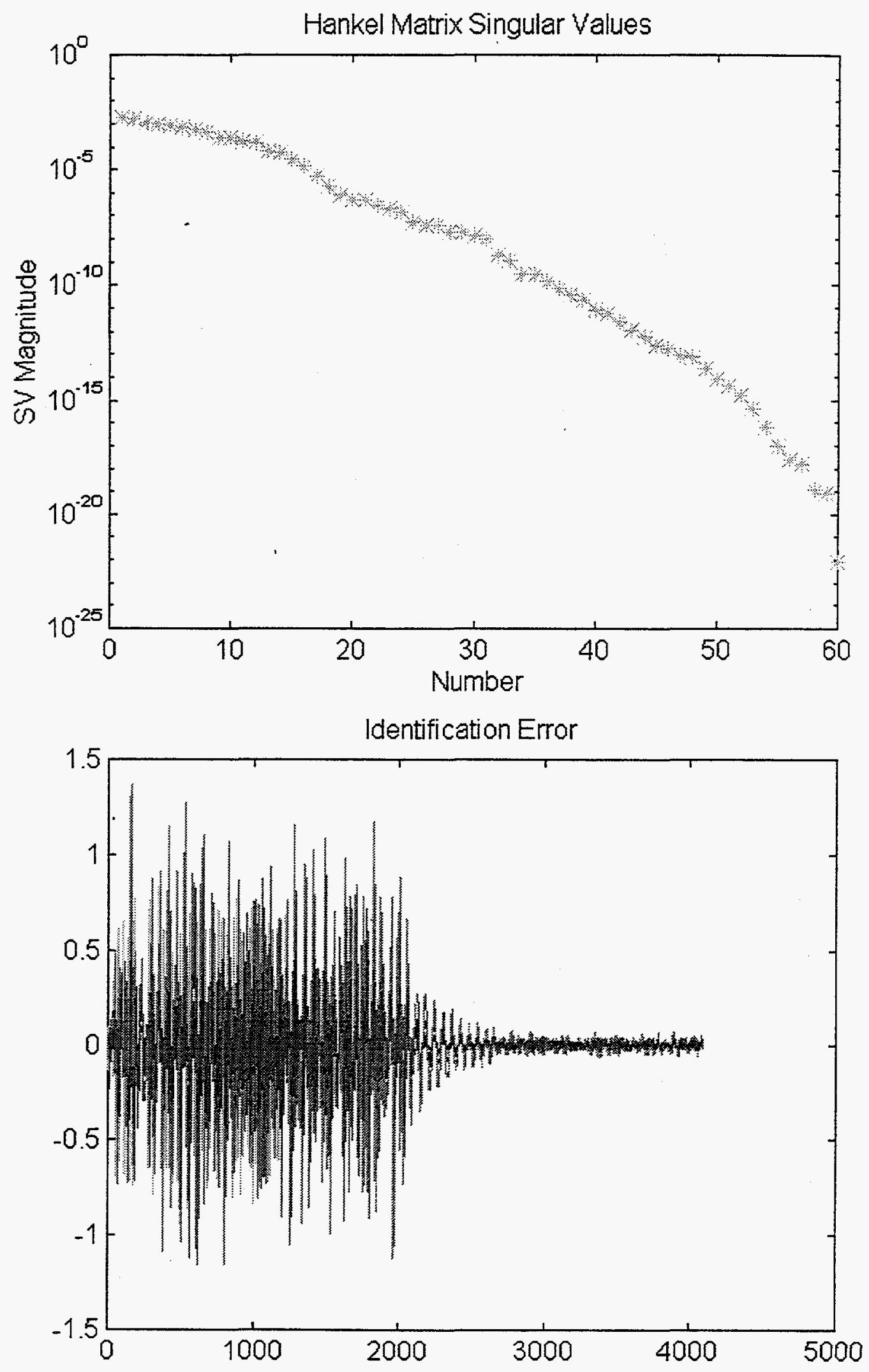
$\gg$ idss2tfr

onr (output $n r$ for freq. resp) $=1$ IDENTIFIED SYSTEM EIGENVALUES

$r I=$

$-6.7991 e+001+2.9467 e+002 i$

$-6.7991 e+001-2.9467 e+002 i$

$-1.7392 e+001+2.7502 e+002 i$

$-1.7392 e+001-2.7502 e+002 i$

$-2.2238 e+001+2.6923 e+002 i$

$-2.2238 e+001-2.6923 e+002 i$

$-9.0534 e+000+2.1370 e+002 i$

$-9.0534 e+000-2.1370 e+002 i$

$-1.9992 e+001+2.0813 e+002 i$

$-1.9992 e+001-2.0813 e+002 i$

$-1.1476 \mathrm{e}+001+1.8402 \mathrm{e}+002 i$

$-1.1476 e+001-1.8402 e+002 i$

$-6.9503 e+000+1.3228 e+002 i$

$-6.9503 e+000-1.3228 e+002 i$

$-9.1418 e+000+1.0940 e+002 i$

$-9.1418 e+000-1.0940 e+002 i$

$-1.3782 e+001+1.0624 e+002 i$

$-1.3782 e+001-1.0624 e+002 i$

$-1.5853 e+001+7.3634 e+001 i$

$-1.5853 e+001-7.3634 e+001 i$

$-9.0060 e+000+6.3330 e+001 i$

$-9.0060 e+000-6.3330 e+001 i$

$-3.7220 e+000+5.2845 e+001 i$

$-3.7220 e+000-5.2845 e+001 i$

$-5.0391 e+000+4.6637 e+001 i$

$-5.0391 e+000-4.6637 e+001 i$

$-1.2882 e+001+3.3725 e+001 i$

$-1.2882 e+001-3.3725 e+001 i$

$-1.4086 \dot{e}+000+1.8290 e+001 i$

$-1.4086 e+000-1.8290 e+001 i$

$-6.7881 e-001+1.0824 e+001 i$

$-6.7881 e-001-1.0824 e+001 i$ 
IDENTIFIED MODAL FREQUENCIES IN $\mathrm{rad} / \mathrm{s}$ AND $\mathrm{Hz}$ $\mathrm{rad} / \mathrm{s} \quad \mathrm{Hz}$

$\mathrm{Er}=$

$3.0242 e+002$

3. $0242 e+002$

$2.7557 e+002$

$2.7557 e+002$

$2.7015 e+002$

$2.7015 e+002$

$2.1390 e+002$

$2.1390 e+002$

$2.0909 e+002$

$2.0909 e+002$

1. $8438 e+002$

$1.8438 e+002$

1. $3246 e+002$

$1.3246 e+002$

$1.0978 \mathrm{e}+002$

$1.0978 e+002$

$1.0713 e+002$

$1.0713 e+002$

$7.5321 e+001$

$7.5321 e+001$

$6.3967 e+001$

$6.3967 e+001$

$5.2976 e+001$

$5.2976 e+001$

$4.6908 e+001$

$4.6908 e+001$

3. $6102 e+001$

3. $6102 \mathrm{e}+001$

$1.8344 \mathrm{e}+001$

$1.8344 e+001$

$1.0845 \mathrm{e}+001$

$1.0845 e+001$
$4.8131 e+001$

$4.8131 e+001$

$4.3859 e+001$

$4.3859 e+001$

4. $2995 \mathrm{e}+001$

$4.2995 e+001$

$3.4043 e+001$

$-3.4043 e+001$

3. $3277 e+001$

3. $3277 e+001$

2. $9344 e+001$

$2.9344 e+001$

$2.1082 \mathrm{e}+001$

$2.1082 e+001$

1. $7472 e+001$

$1.7472 \mathrm{e}+001$

$1.7051 e+001$

$1.7051 \mathrm{e}+001$

1. $1988 e+001$

$1.1988 e+001$

$1.0181 e+001$

$1.0181 e+001$

$8.4315 e+000$

$8.4315 e+000$

$7.4656 e+000$

$7.4656 e+000$

$5.7458 e+000$

$5.7458 e+000$

$2.9196 e+000$

$2.9196 e+000$

$1.7260 \mathrm{e}+000$

$1.7260 \mathrm{e}+000$ 
IDENTIFIED SYSTEM ZEROS RELATED TO THE OUTPUT 1 $\mathrm{ZER}=$

$-8.2677 e+003$

$-9.3644 e+000+2.7448 e+002 i$

$-9.3644 e+000-2.7448 e+002 i$

$-2.2283 e+001+2.6726 e+002 i$

$-2.2283 e+001-2.6726 e+002 i$

$-6.5613 e+000+2.1482 e+002 i$

$-6.5613 e+000-2.1482 e+002 i$

$-1.2435 e+001+2.0962 e+002 i$

$-1.2435 e+001-2.0962 e+002 i$

$-1.6330 e+001+1.8601 e+002 i$

$-1.6330 e+001-1.8601 e+002 i$

$-1.0576 e+001+1.3504 e+002 i$

$-1.0576 e+001-1.3504 e+002 i$

$-6.8961 e+000+1.1142 e+002 i$

$-6.8961 e+000-1.1142 e+002 i$

$-9.6600 e+000+9.5263 e+001 i$

$-9.6600 e+000-9.5263 e+001 i$

$-7.8338 e+000+7.7705 e+001 i$

$-7.8338 e+000-7.7705 e+001 i$

$-8.1881 e+000+6.0737 e+001 i$

$-8.1881 e+000-6.0737 e+001 i$

$-4.3657 e+000+5.0886 e+001 i$

$-4.3657 e+000-5.0886 e+001 i$

$-6.6433 e+000+4.5538 e+001 i$

$-6.6433 e+000-4.5538 e+001 i$

$-1.6366 e+001+3.6220 e+001 i$

$-1.6366 e+001-3.6220 e+001 i$

$-2.0061 e+000+1.7830 e+001 i$

$-2.0061 e+000-1.7830 e+001 i$

$-4.4938 e+000+1.3163 e+001 i$

$-4.4938 e+000-1.3163 e+001 i$

$3.1822 e+000$ 

$\begin{array}{cc}\text { IDENTIFIED ZERO FREQUENCIES IN } \mathrm{rad} / \mathrm{s} \text { AND } \mathrm{Hz} \\ \mathrm{rad} / \mathrm{s} & \mathrm{Hz}\end{array}$

$\mathrm{zfr}=$

$8.2677 e+003 \quad 1.3158 e+003$

$2.7464 \mathrm{e}+002 \quad 4.3710 \mathrm{e}+001$

$2.7464 e+002 \quad 4.3710 e+001$

$2.6819 e+002 \quad 4.2683 e+001$

$2.6819 e+002 \quad 4.2683 e+001$

$2.1492 \mathrm{e}+002 \quad 3.4206 \mathrm{e}+001$

$2.1492 e+002 \quad .3 .4206 e+001$

$2.0999 e+002 \quad 3.3421 e+001$

$2.0999 e+002 \quad 3.3421 e+001$

$1.8672 e+002 \quad 2.9718 e+001$

$1.8672 e+002 \quad 2.9718 e+001$

$1.3546 e+002 \quad 2.1559 e+001$

$1.3546 e+002 \quad 2.1559 e+001$

$1.1164 e+002 \quad 1.7767 e+001$

$1.1164 e+002 \quad 1.7767 e+001$

$9.5752 e+001 \quad 1.5239 e+001$

$9.5752 e+001 \quad 1.5239 e+001$

$7.8099 e+001 \quad 1.2430 e+001$

$7.8099 e+001 \quad 1.2430 e+001$

$6.1286 e+001 \quad 9.7540 e+000$

$6.1286 e+001 \quad 9.7540 e+000$

$5.1073 e+001 \quad 8.1285 e+000$

$5.1073 e+001 \quad 8.1285 e+000$

$4.6020 e+001 \quad 7.3243 e+000$

$4.6020 e+001 \quad 7.3243 e+000$

$3.9746 e+001 \quad 6.3257 e+000$

$3.9746 e+001 \quad 6.3257 e+000$

$1.7942 e+001 \quad 2.8556 e+000$

$1.7942 e+001 \cdot 2.8556 e+000$

$1.3909 e+001 \quad 2.2137 e+000$

$1.3909 e+001 \quad 2.2137 e+000$

$3.1822 e+000 \quad 5.0647 e-001$

Frequency response for OUTPUT 1 is now displayed

in the Figure Window.

If you want to obtain frequency response for more than one output, run idss2tfr.m again and/or run zoomtfr.m to look at a specified frequency range. 

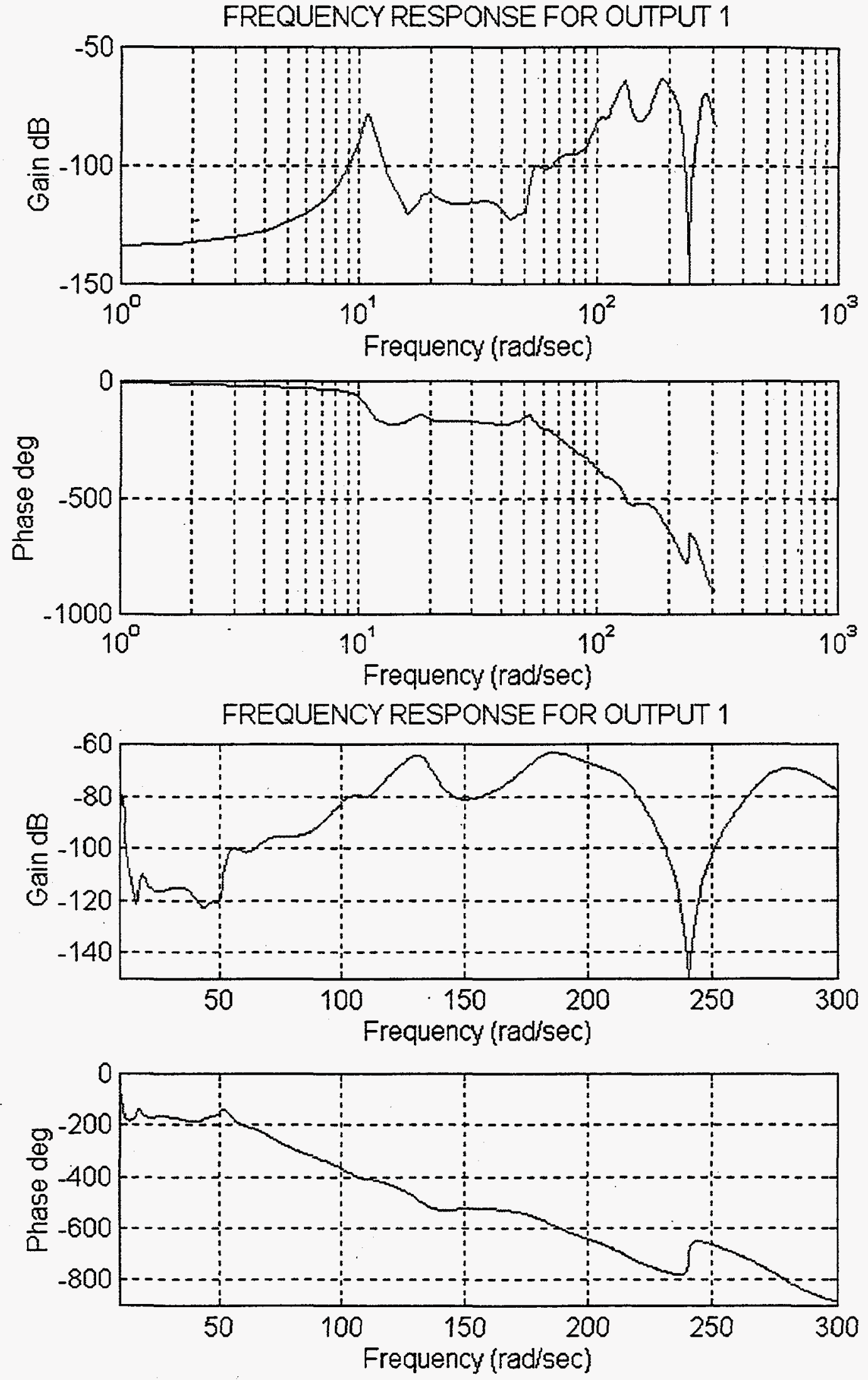
Frequency Response for OUTPUT2

$\gg$ idss2tfr

onr(output $n r$ for freq. resp) $=2$

IDENTIFIED SYSTEM ZEROS RELATED TO THE OUTPUT 2 ZER $=$

$-5.3409 e+003$

$-2.0838 e+001+2.8955 e+002 i$

$-2.0838 e+001-2.8955 e+002 i$

$-1.2218 e+001+2.7112 e+002 i$

$-1.2218 e+001-2.7112 e+002 i$

$-1.2387 e+001+2.1254 e+002 i$

$-1.2387 e+001-2.1254 e+002 i$

$1.1209 e+001+2.0471 e+002 i$

$1.1209 e+001-2.0471 e+002 i$

$-1.3734 e+001+2.0001 e+002 i$

$-1.3734 e+001-2.0001 e+002 i$

$-9.4953 e+000+1.4900 e+002 i$

$-9.4953 e+000-1.4900 e+002 i$

$-2.5113 e+001+1.1535 e+002 i$

$-2.5113 e+001-1.1535 e+002 i$

$-7.9257 e+000+1.1138 e+002 i$

$-7.9257 e+000-1.1138 e+002 i$

$1.8217 e+001+7.3335 e+001 i$

$1.8217 e+001-7.3335 e+001 i$

$-2.6717 e+001+7.5228 \mathrm{e}+001 i$

$-2.6717 e+001-7.5228 e+001 i$

$-1.0392 e+001+6.1185 e+001 i$

$-1.0392 e+001-6.1185 e+001 i$

$-4.4618 e+000+4.7887 e+001 i$

$-4.4618 e+000-4.7887 e+001 i$

$-8.2358 e+000+4.5898 e+001 i$

$-8.2358 e+000-4.5898 e+001 i$

$-2.5918 e+001+9.2964 e+000 i$

$-2.5918 e+001-9.2964 e+000 i$

$-1.1422 e+000+1.7087 e+001 i$

$-1.1422 e+000-1.7087 e+001 i$

$9.8454 \mathrm{e}+000$ 


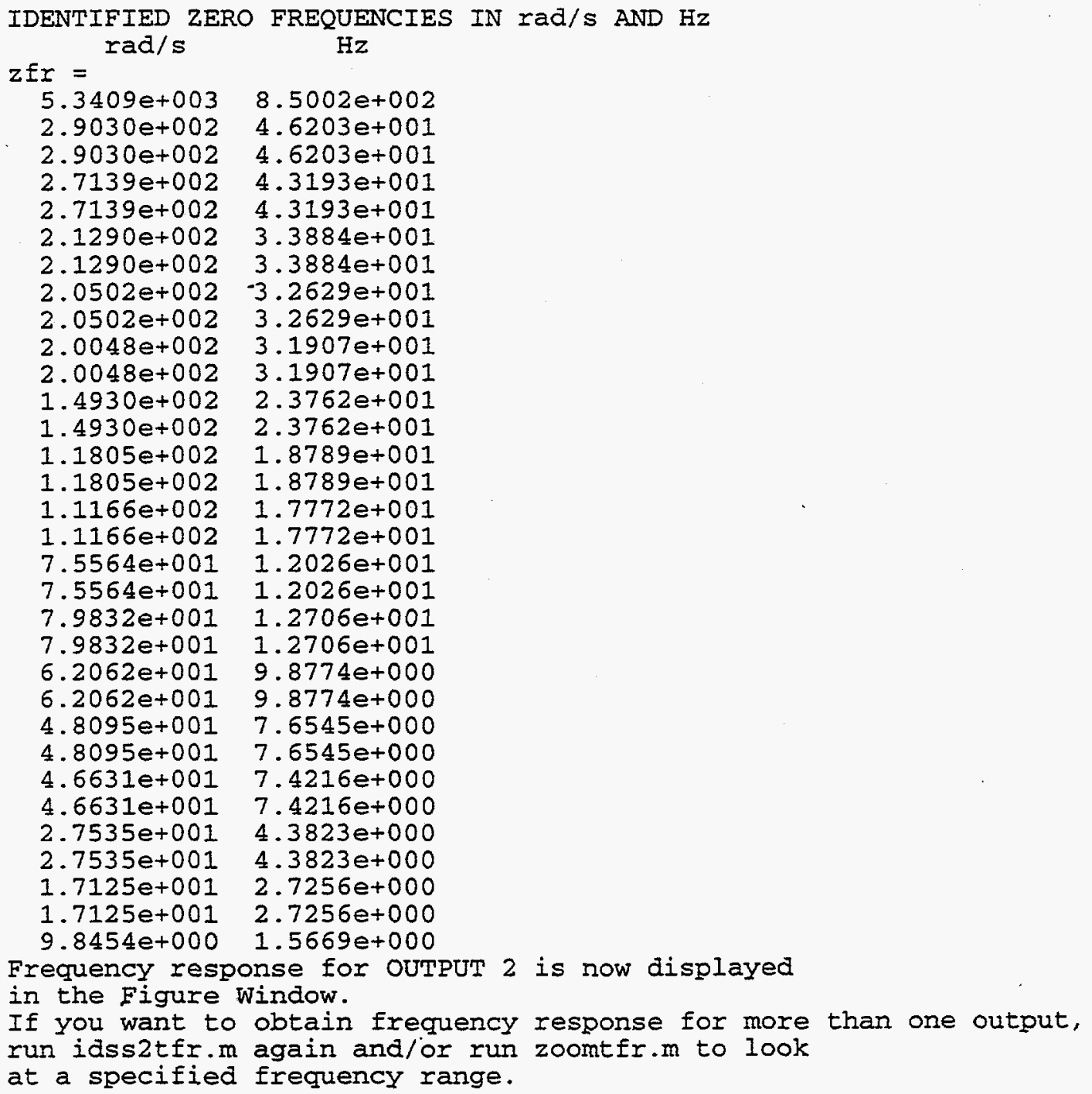


FREQUENCY RESPONSE FOR OUTPUT 2
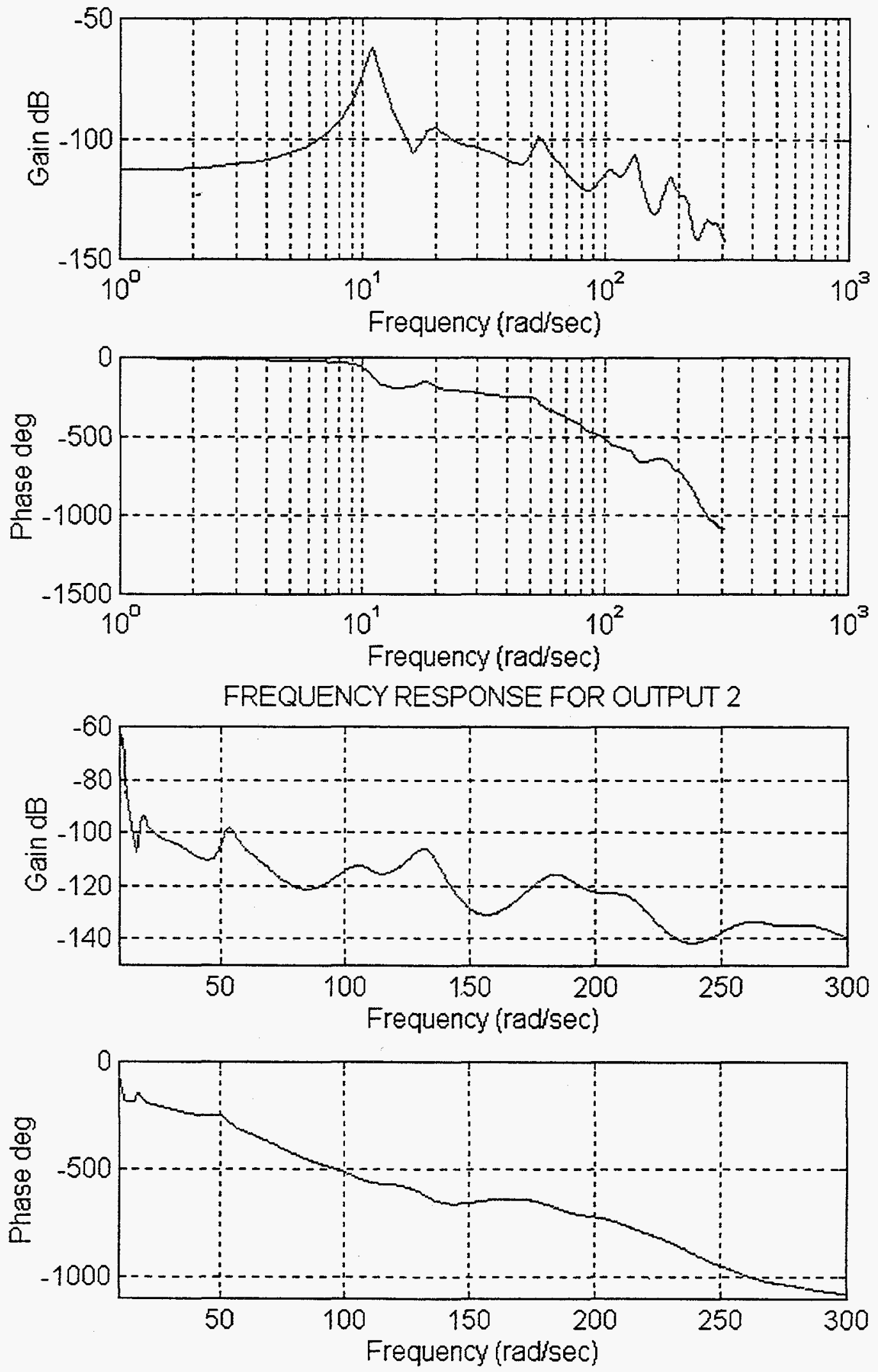


\section{Frequency Response for OUTPUT3}

idss2tfr

onr (output nr for freq. resp) $=3$

IDENTIFIED SYSTEM ZEROS RELATED TO THE OUTPUT 3

$\mathrm{ZER}=$

$-5.1048 e+004$

$-1.0570 e+001+2.7420 e+002 i$

$-1.0570 e+001-2.7420 e+002 i$

$-2.9894 e+001+2.5331 e+002 i$

$-2.9894 e+001-2.5331 e+002 i$

$-1.2847 e+001+2.2567 e+002 i$

$-1.2847 e+001-2.2567 e+002 i$

$-1.5584 e+001+2.1145 e+002 i$

$-1.5584 e+001-2.1145 e+002 i$

$-3.9626 e+001+1.8612 e+002 i$

$-3.9626 e+001-1.8612 e+002 i$

$5.7588 e+001+1.3913 e+002 i$

$5.7588 e+001-1.3913 e+002 i$

$-2.9048 e+001+1.3844 e+002 i$

$-2.9048 e+001-1.3844 e+002 i$

$-7.5063 e+000+1.1155 e+002 i$

$-7.5063 e+000-1.1155 e+002 i$

$1.8775 e+001+7.5839 e+001 i$

$1.8775 e+001-7.5839 e+001 i$

$-2.9924 e+001+7.3913 e+001 i$

$-2.9924 e+001-7.3913 e+001 i$

$-1.0441 e+001+6.1182 e+001 i$

$-1.0441 e+001-6.1182 e+001 i$

$4.3659 e+001$

$-8.2001 e+000+4.5852 e+001 i$

$-8.2001 e+000-4.5852 e+001 i$

$-2.9736 e+001+1.5881 e+001 i$

$-2.9736 e+001-1.5881 e+001 i$

$-1.8093 e+000+2.1812 e+001 i$

$-1.8093 e+000-2.1812 e+001 i$

$-2.3112 e+001$

$1.0454 \mathrm{e}+001$ 


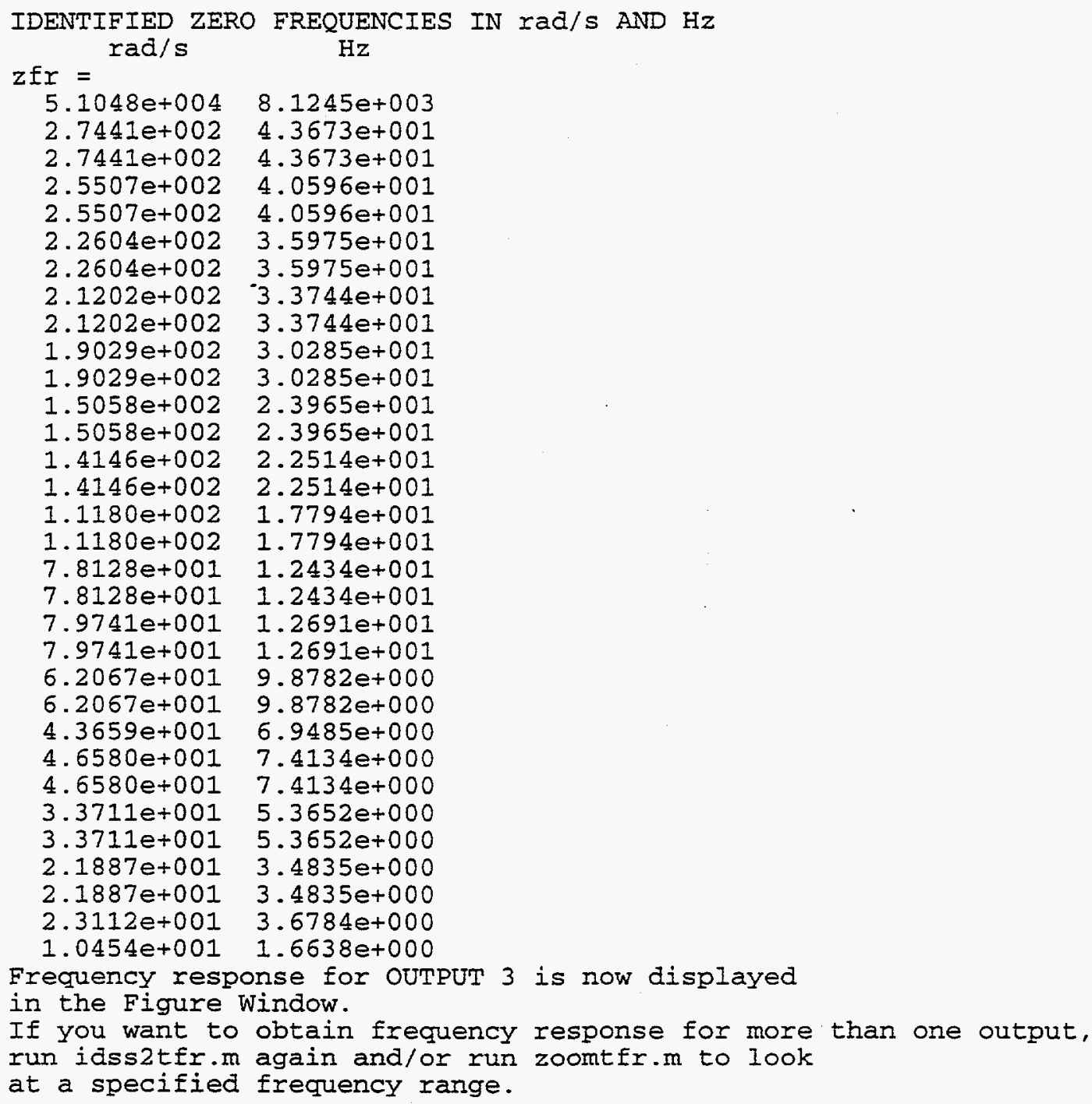


FREQUENCY RESPONSE FOR OUTPUT 3
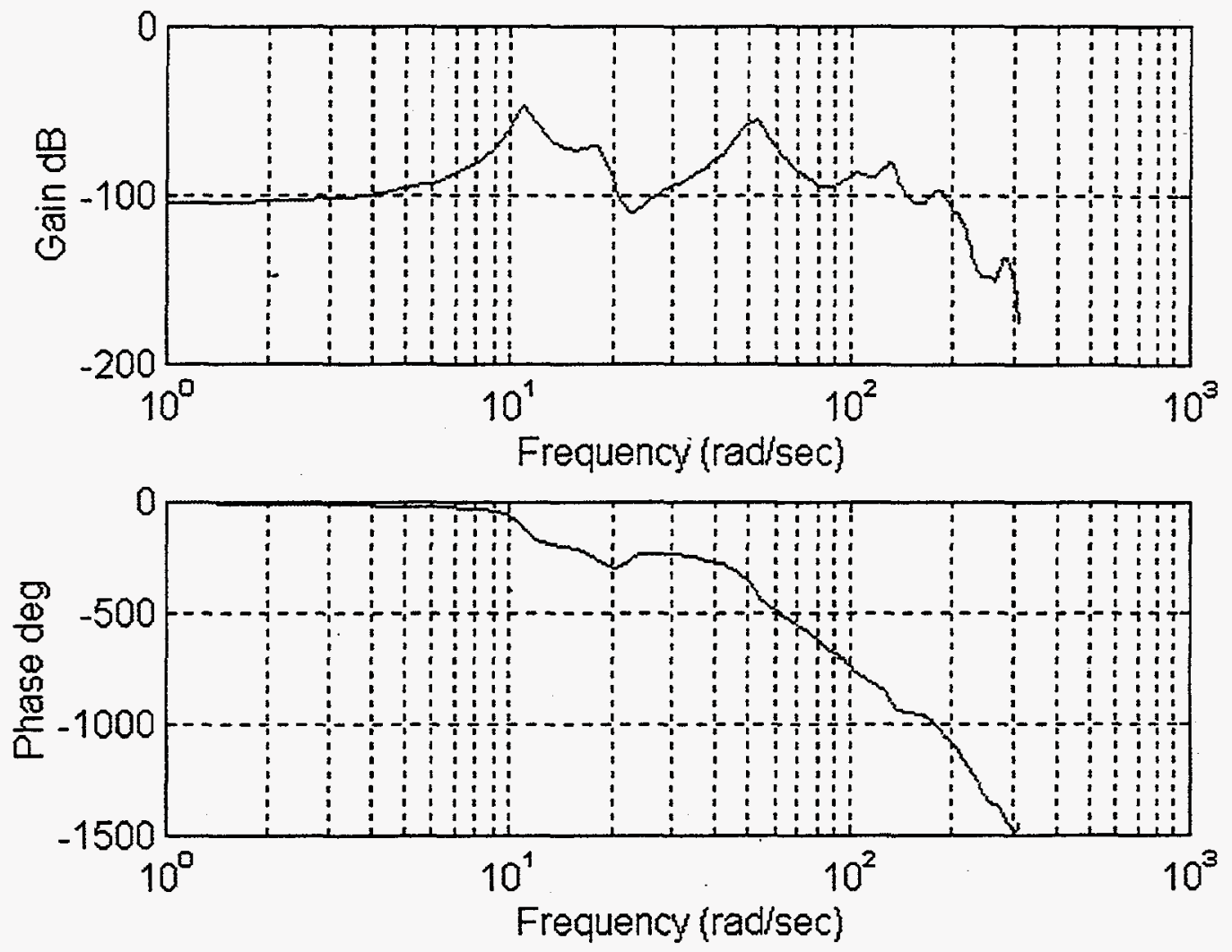

FREQUENCY RESPONSE FOR OUTPUT 3
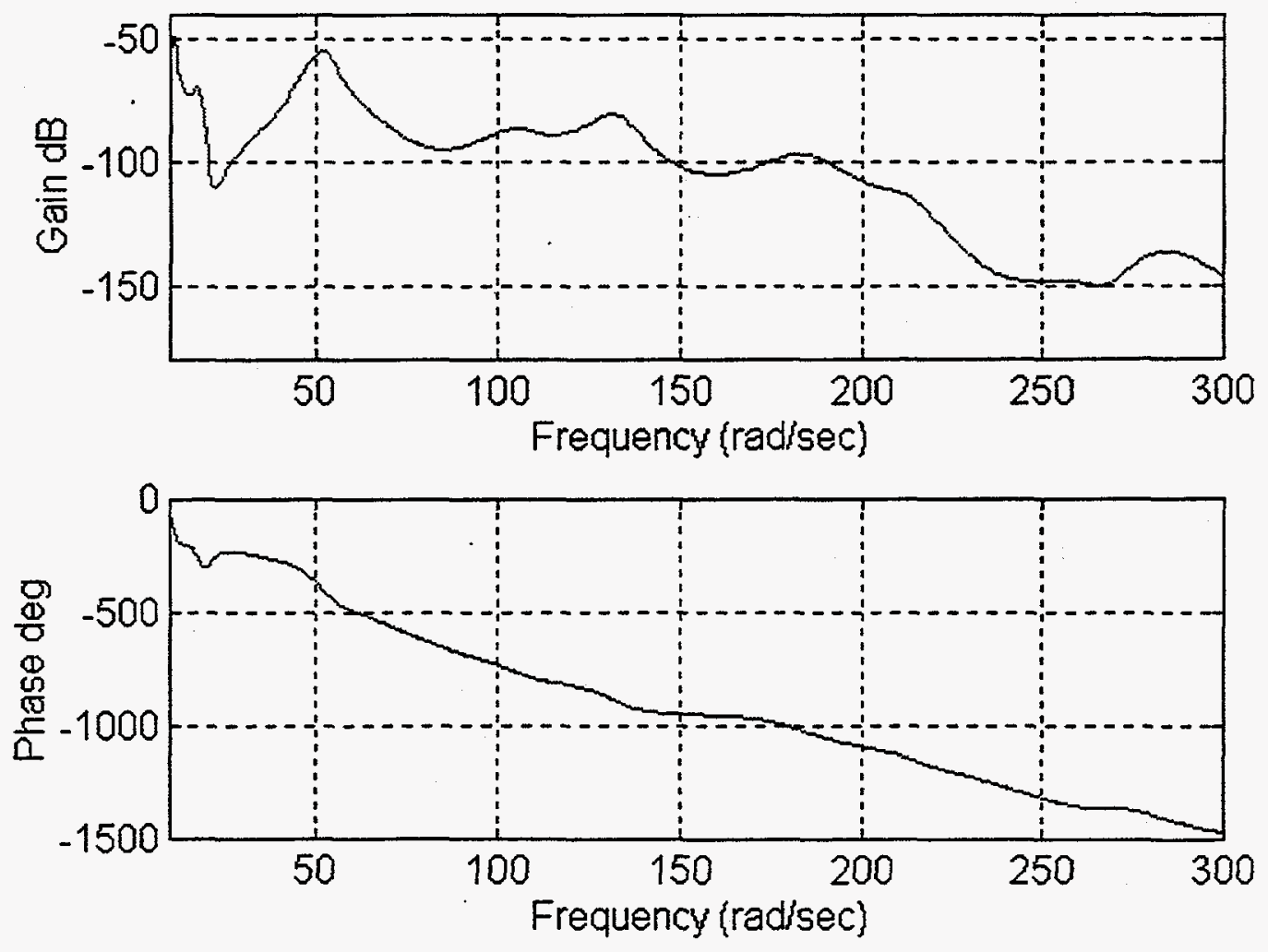


\title{
Frequency Response for OUTPUT4
}

\author{
IDENTIFIED SYSTEM ZEROS RELATED TO THE OUTPUT 4 \\ $\mathrm{ZER}=$ \\ $-4.4647 e+004$ \\ $-1.6711 e+001+2.7746 e+002 i$ \\ $-1.6711 e+001-2.7746 e+002 i$ \\ $-1.8305 e+001+2.6483 e+002 i$ \\ $-1.8305 e+001-2.6483 e+002 i$ \\ $-8.8802 e+000+2.1348 e+002 i$ \\ $-8.8802 e+000-2.1348 e+002 i$ \\ $-1.8125 e+001+2.0942 e+002 i$ \\ $-1.8125 e+001-2.0942 e+002 i$ \\ $-9.0595 e+000+1.8403 e+002 i$ \\ $-9.0595 e+000-1.8403 e+002 i$ \\ $-1.9401 e+001+1.3936 e+002 i$ \\ $-1.9401 e+001-1.3936 e+002 i$ \\ $7.9153 e+000+1.2416 e+002 i$ \\ $7.9153 e+000-1.2416 e+002 i$ \\ $-7.4000 e+000+1.1196 e+002 i$ \\ $-7.4000 e+000-1.1196 e+002 i$ \\ $5.7863 e+001$ \\ $1.6192 e+001+7.3881 e+001 i$ \\ $1.6192 e+001-7.3881 e+001 i$ \\ $-2.8465 e+001+7.1926 e+001 i$ \\ $-2.8465 e+001-7.1926 e+001 i$ \\ $-1.0329 e+001+6.0996 e+001 i$ \\ $-1.0329 e+001-6.0996 e+001 i$ \\ $-5.3618 e+001$ \\ $-8.2044 e+000+4.5841 e+001 i$ \\ $-8.2044 e+000-4.5841 e+001 i$ \\ $-2.5938 e+001+1.1220 e+001 i$ \\ $-2.5938 e+001-1.1220 e+001 i$ \\ $1.2226 e+001$ \\ $-2.4945 e-001+5.0606 e+000 i$ \\ $-2.4945 e-001-5.0606 e+000 i$
}




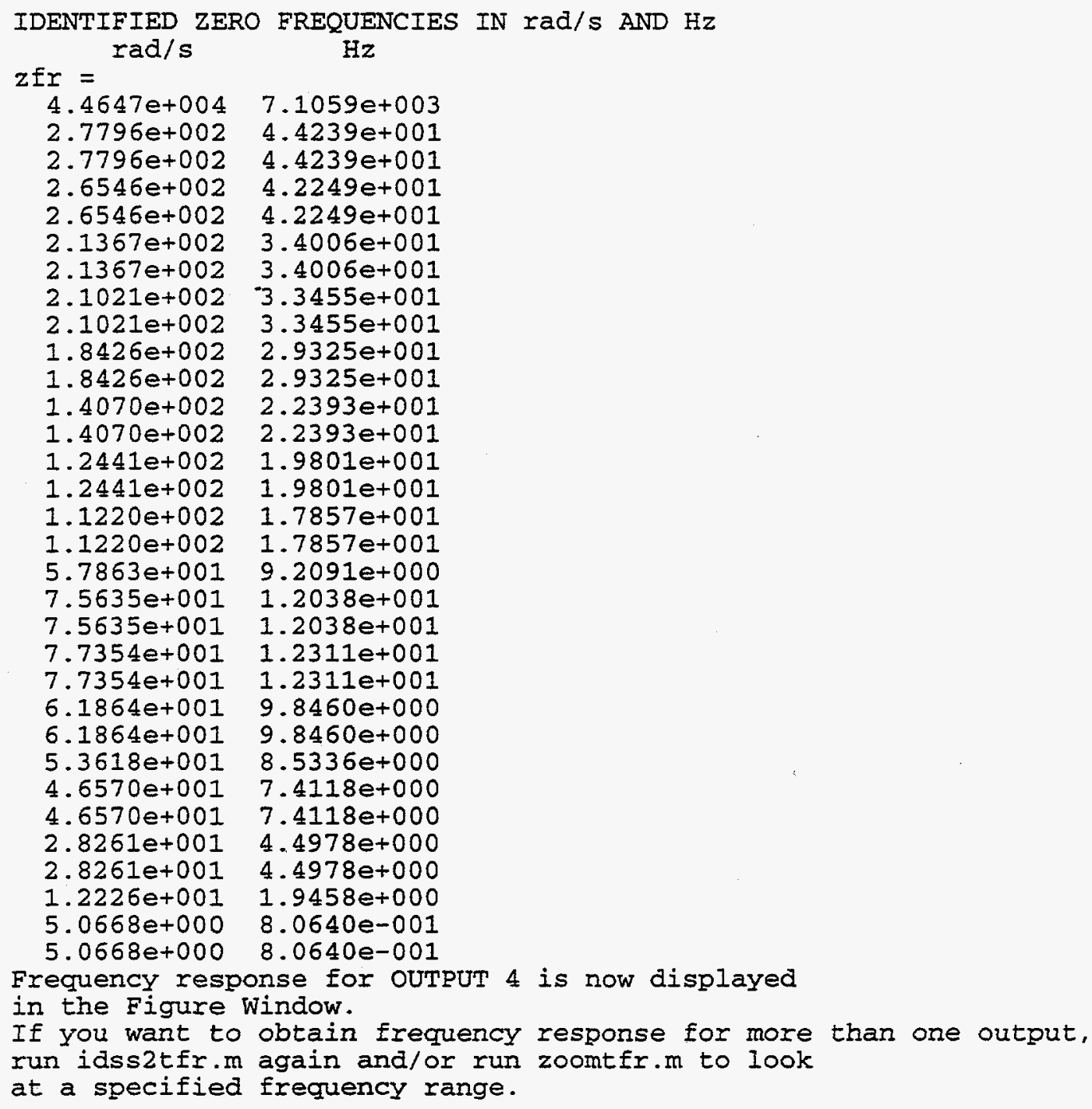



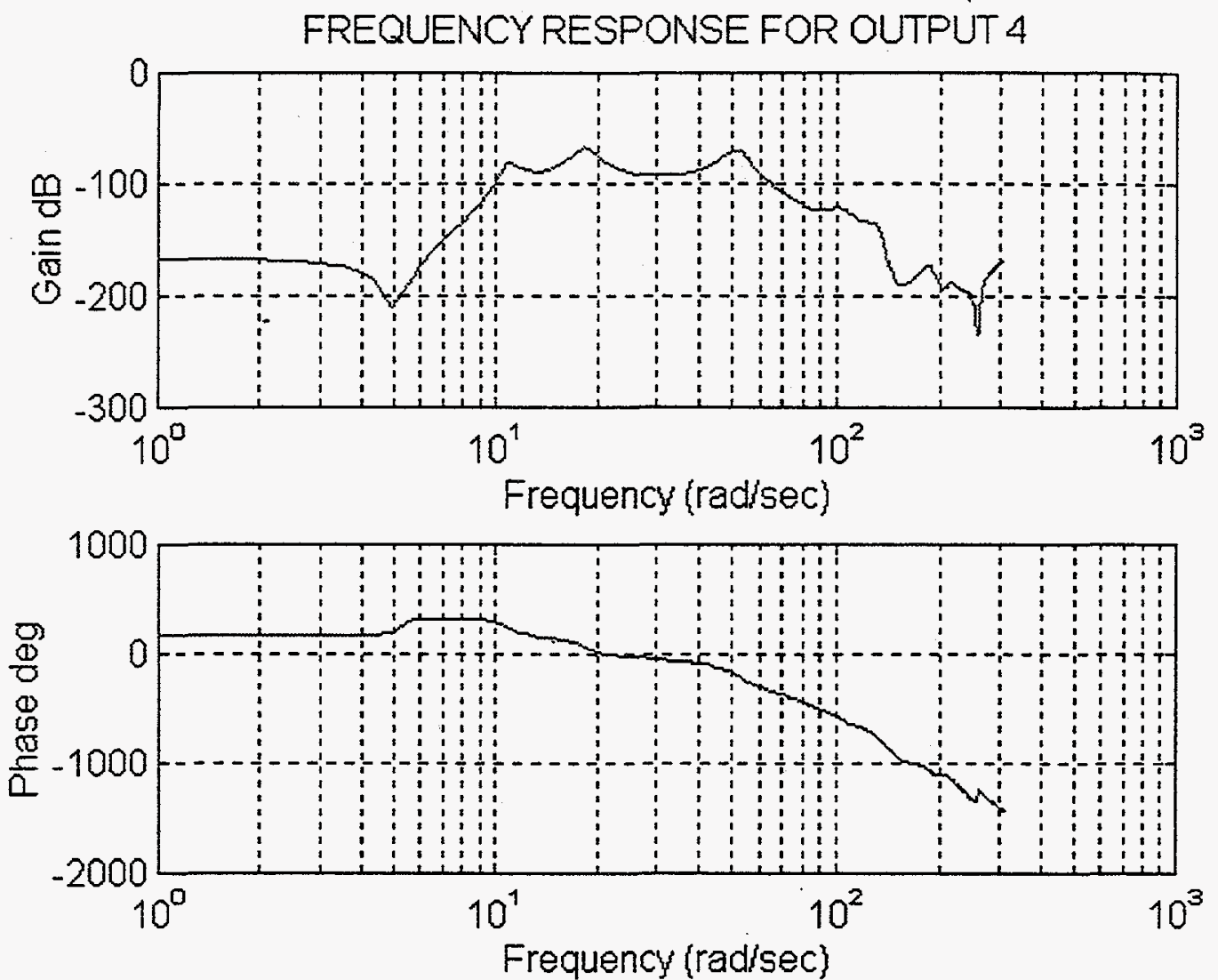

FREQUENCY RESPONSE FOR OUTPUT 4
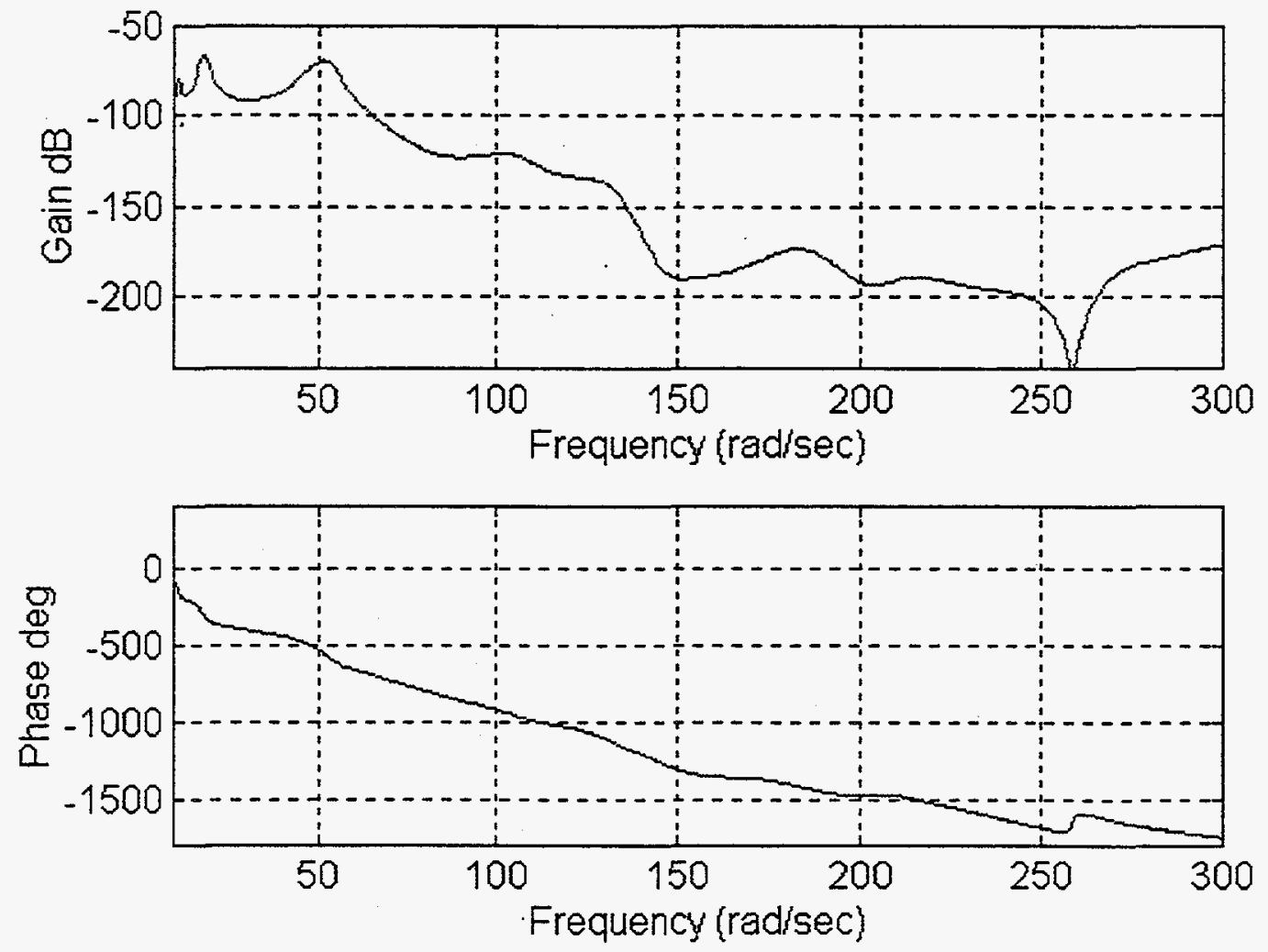


\section{Frequency Response for OUTPUT5}

$\gg \operatorname{idss} 2 t f r$

onr (output nr for freq. resp) $=5$

IDENTIFIED SYSTEM ZEROS RELATED TO THE OUTPUT 5 $\mathrm{ZER}=$

$-4.9015 e+004$

$-1.6382 e+001+2.7758 e+002 i$

$-1.6382 e+001-2.7758 e+002 i$

$-1.7745 e+001+2.6301 e+002 i$

$-1.7745 e+001-2.6301 e+002 i$

$-9.6086 e+000+2.1294 e+002 i$

$-9.6086 e+000-2.1294 e+002 i$

$-1.9802 e+001+2.0830 e+002 i$

$-1.9802 e+001-2.0830 e+002 i$

$3.5522 e+000+1.8161 e+002 i$

$3.5522 \mathrm{e}+000-1.8161 \mathrm{e}+002 i$

$-1.9656 e+001+1.4696 e+002 i$

$-1.9656 e+001-1.4696 e+002 i$

$-1.0215 e+002$

$-2.7344 e+000+1.1935 e+002 i$

$-2.7344 e+000-1.1935 e+002 i$

$-7.3819 e+000+1.1213 e+002 i$

$-7.3819 e+000-1.1213 e+002 i$

$7.4013 e+001$

$1.6761 e+001+7.5462 e+001 i$

$1.6761 \mathrm{e}+001-7.5462 \mathrm{e}+001 i$

$-2.7773 e+001+7.0537 e+001 i$

$-2.7773 e+001-7.0537 e+001 i$

$-1.0883 e+001+6.1275 e+001 i$

$-1.0883 e+001-6.1275 e+001 i$

$-8.1574 e+000+4.5751 e+001 i$

$-8.1574 e+000-4.5751 e+001 i$

$-2.2862 e+001+1.1066 e+001 i$

$-2.2862 e+001-1.1066 e+001 i$

$1.1535 \mathrm{e}+001$

$-2.4699 e-001+5.0580 e+000 i$.

$-2.4699 e-001-5.0580 e+000 i$ 


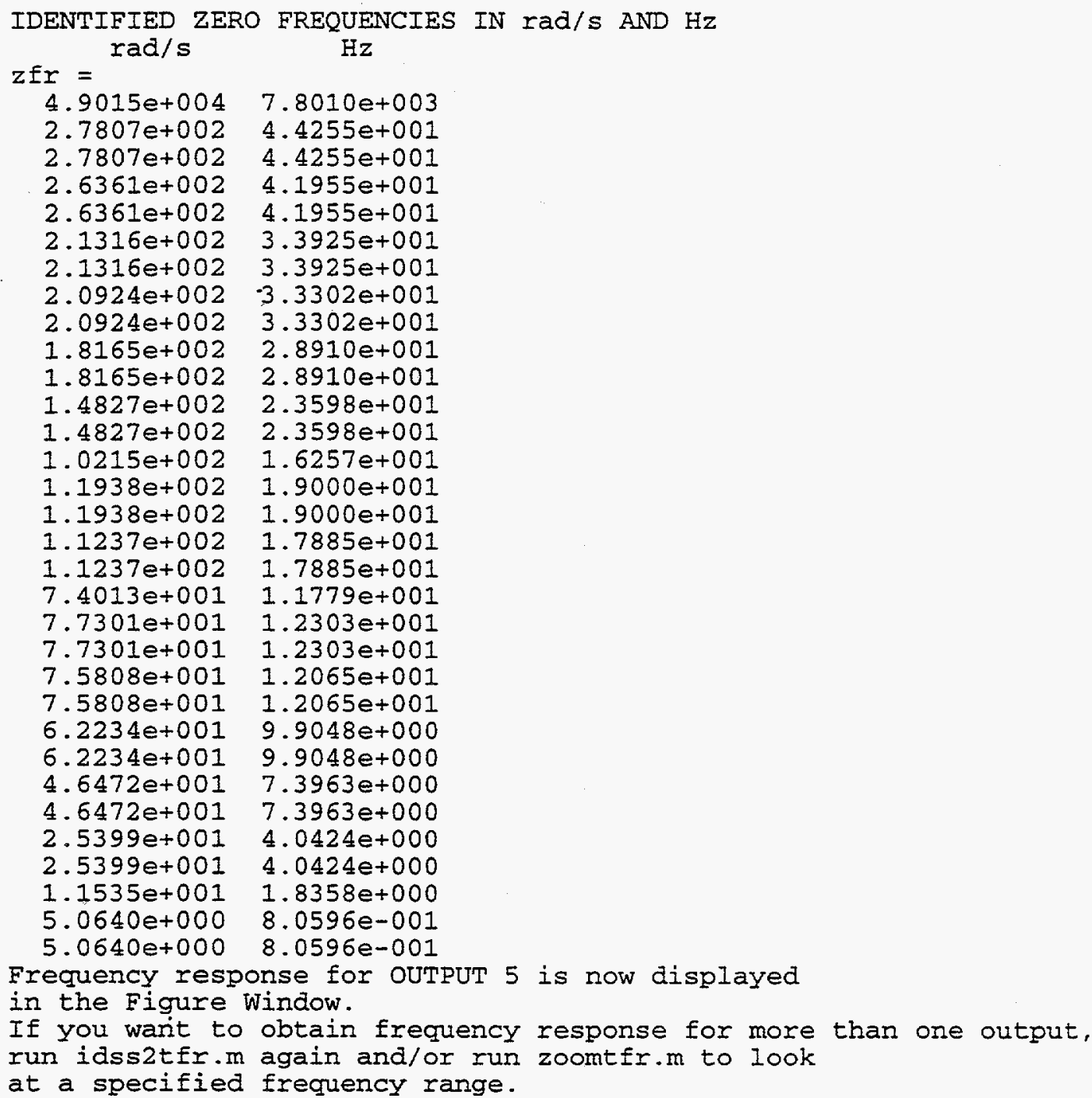



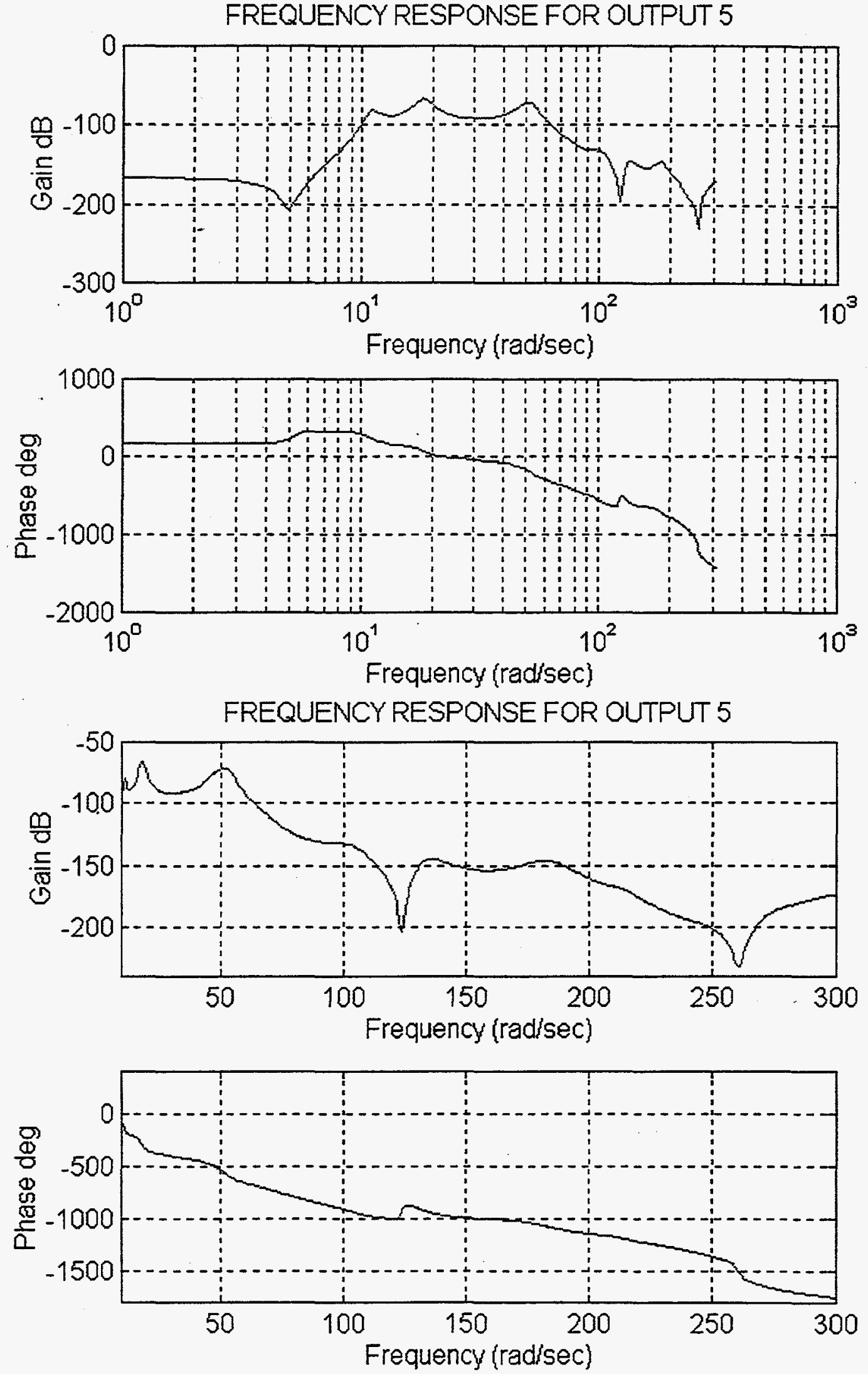


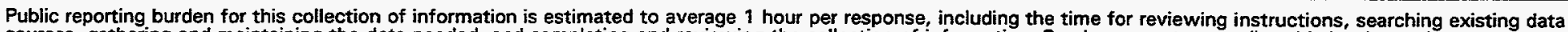

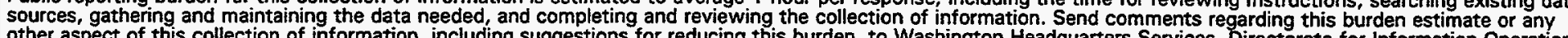

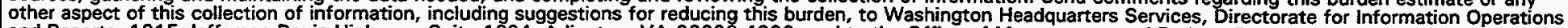

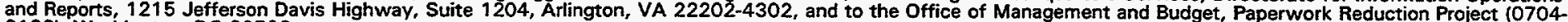
$0188)$, Washington, DC 20503.

\begin{tabular}{l|l|l} 
1. & $\begin{array}{c}\text { 2. REPORT DATE } \\
\text { July } 1995\end{array}$ & $\begin{array}{l}\text { 3. REPORT TYPE AND DATES COVERED } \\
\text { Subcontract Report }\end{array}$ \\
\hline
\end{tabular}

\section{TITLE AND SUBTITLE}

Development of an Advanced System Identification Technique for Comparing ADAMS Analytical Results with Modal Test Data for a Micon 65/13 Wind Turbine

6. AUTHOR(S)

Jan T. Bialasiewicz

7. PERFORMING ORGANIZATION NAME(S) AND ADDRESS(ES)

Jan T. Bialasiewicz
5. FUNDING NUMBERS

C: CAT-5-15011-01

TA: WE518210
8. PERFORMING ORGANIZATION REPORT NUMBER
9. SPONSORING/MONITORING AGENCY NAME(S) AND ADDRESS(ES)

National Renewable Energy Laboratory

1617 Cole Blvd.

Golden, CO 80401-3393
10. SPONSORING/MONITORING AGENCY REPORT NUMBER

TP-442-7813

DE95009260

11. SUPPLEMENTARY NOTES

NREL Technical Monitor: Richard M. Osgood (303) 384-6949

12a. DISTRIBUTION/AVAILABILITY STATEMENT

National Technical Information Service

U.S. Department of Commerce

5285 Port Royal Road

Springfield, VA 22161 12b. DISTRIBUTION CODE

UC-1213

\section{ABSTRACT (Maximum 200 words)}

14. SUBJECT TERMS wind turbine design

17. SECURITY CLASSIFICATION OF REPORT

Unclassified
18. SECURITY CLASSIFICATION OF THIS PAGE Unclassified
19. SECURITY CLASSIFICATION OF ABSTRACT

Unclassified
15. NUMBER OF PAGES

16. PRICE CODE

20. LIMITATION OF ABSTRACT

UL

Standard Form 298 (Rev. 2-89) Prescribed by ANSI Std. 239-18 